\title{
Optical-Router-Based Dynamically Reconfigurable Photonic Access Network
}

\author{
Rajeev Roy
}




\title{
Samenstelling van de promotiecommissie
}

\author{
Voorzitter \& secretaris: \\ prof.dr.ir. A.J. Mouthaan \\ University of Twente, the Netherlands \\ Promotor: \\ prof.dr.ir. W. van Etten \\ University of Twente, the Netherlands

\section{Leden:} \\ prof.dr.ir J.M. Dumas \\ Université de Limoges, France \\ prof. dr. W.G. Scanlon \\ University of Twente, the Netherlands \\ prof.dr.ir C.H. Slump \\ University of Twente, the Netherlands \\ prof.dr.ir G.J.M. Smit \\ University of Twente, the Netherlands \\ dr.ir. H. de Waardt \\ Technical University of Eindhoven, the Netherlands \\ dr.ir G. van den Hoven \\ Genexis B.V, the Netherlands
}

The work is funded by the Dutch Ministry of Economic Affairs through the BSIK Freeband Broadband Photonics project under contract BSIK 03025.

The research work presented in this thesis was carried out at the Telecommunication Engineering group, Faculty of Electrical Engineering, Mathematics and Computer Science, University of Twente P.O Box 217, 7500AE Enschede, the Netherlands

The front cover depicts workers installing a fiber duct in a village close to Bandipur, India

Copyright (C 2014 by Rajeev Roy

All rights reserved. No part of this publication may be reproduced, stored in a retrieval system, or transmitted, in any form or by any means, electronic, mechanical, photocopying, recording, or otherwise, without the prior written consent of the copyright owner.

ISBN: 978-94-6191-428-6

Printed by Ipskamp Drukkers B.V., Enschede, the Netherlands 


\title{
OPTICAL-ROUTER-BASED DYNAMICALLY RECONFIGURABLE PHOTONIC ACCESS NETWORK
}

\section{PROEFSCHRIFT}

\author{
ter verkrijging van \\ de graad van doctor aan de Universiteit Twente, \\ op gezag van de rector magnificus, \\ prof.dr. H. Brinksma, \\ volgens besluit van het College voor Promoties \\ in het openbaar te verdedigen \\ op woensdag 8 januari 2014 om 16:45 uur
}

door

Rajeev Roy

geboren op 11 augustus 1973

te New Delhi, India 
Dit proefschrift is goedgekeurd door:

De promotor:

prof.dr.ir. W. van Etten 
For my loved ones 



\section{Abstract}

The Broadband photonics (BBP) project under the Freeband consortium of projects investigated the design of a dynamically reconfigurable photonic access network. Access networks form a key link in ensuring optimal bandwidth to the end user without which any improvements deeper in the network in the aggregation or core segments are mitigated.

Optical fiber deployment in the access are a natural choice as the bandwidth demands increase and Passive Optical Networks (PONs) form a preferred way of implementing fiber deployments. PONs have a logical Point to Multi-Point (P2MP) topology where in the downstream, the Optical Line Termination (OLT) broadcasts to all Optical Network Units (ONUs) while in the upstream, it is a many-toone transmission and is contention based. Time Division Multiplexing (TDM) access is one way of arbitration and in this thesis a qualitative analysis and comparison of the leading TDM based PON standards- EPON and GPON is presented.

In traditional PONs, the average bandwidth available per user depends on the number of ONUs supported by a single OLT. Typically the OLT-ONU association is fixed. The BBP network concept extends the view of a fiber plant deployment to a stack of logical PONs where multiple wavelength pairs are used to support a number of "logical" PONs. In this network, a headend based OLT transmits the downstream data by modulating a C-Band, Dense Wavelength Division Multiplexed (DWDM) laser. In addition it transmits the light of a Continuous Wave (CW) DWDM laser, also in the C-Band, which is modulated with data by a Reflective Semiconductor Optical Amplifier (RSOA) in an ONU and used for upstream communication. The ONUs are thus wavelength agnostic.

The OLT-ONU association is thus dynamic and depends on the wavelength pair added/dropped towards the ONU. The bandwidth availability to an end user can be optimised on an inter-PON basis. The research work focuses on the techniques to 
realise the bandwidth optimisation using Linear Programming techniques and describes the service delivery architecture that can be realised to ensure an optical service delivery to the end user using conventional protocols of operation. The perspective views of the network from a data to control plane operation are also presented.

Rajeev Roy 


\section{Samenvatting (Abstract in Dutch)}

Het Broadband Photonics (BPP) project, als onderdeel van het Freeband consortium van projecten, heeft onderzoek gedaan naar de ontwikkeling van een dynamisch herconfigureerbaar photonic access-netwerk. Access-netwerken vormen een belangrijke schakel in de keten die optimale bandbreedte naar de abonnee moet kunnen garanderen. Verbeteringen dieper in het netwerk zijn nutteloos, als er geen verbetering wordt aangebracht in het access-network.

Omdat de bandbreedte-eisen steeds hoger worden, is de uitrol van optische accessnetwerken een logische keuze, en Passive Optical Networks (PONs) vormen de meest geschikte implementatie daarvan. PON's hebben een logische "Point-toMulti-Point (P2MP)"-topologie, waarbij de zogeheten Optical Line Termination (OLT) in de neerwaartse richting uitzendt naar alle Optical Network Units (ONUs), terwijl in de opwaartse richting sprake is van "many-to-one"-transmissie, en deze is gebaseerd op de inhoud. "Time Division Multiplexing (TDM)"-access is een mogelijke manier van arbitrage. In dit proefschrift wordt een kwalitatief overzicht gepresenteerd van de meest gebruikte TDM-gebaseerde PONstandaarden, te weten EPON en GPON.

In traditionele PON's is de gemiddelde beschikbare bandbreedte per gebruiker afhankelijk van het aantal ONU's dat wordt ondersteund door een enkele OLT. In het algemeen ligt deze verhouding vast. Het BBP-netwerkconcept verlegt het principe van een gegeven glasvezelnetwerk naar een verzameling van logische PON's, waarbij meerdere golflengte-paren worden gebruikt om meerdere "logische PONs" te creëren. In dit netwerk stuurt de OLT de neerwaartse informatie op een Dense Wavelength Division Multiplexing (DWDM) laser. Hiervoor wordt een laser in de C-Band gebruikt. Daarnaast stuurt de OLT licht van een ongemoduleerde laser eveneens neerwaarts in de C-Band. Dit licht wordt door golflengte- agnostische ONUs gemoduleerd voor opwaartse communicatie. Die modulator hiervoor is gebaseerd op een "Reflective Semiconductor Optical Amplifier (RSOA"). 
De OLT/ONU-associatie is dus dynamisch en deze is afhankelijk van het aantal beschikbare golflengte-paren richting de ONU. De beschikbare bandbreedte naar de abonnee kan zodoende worden geoptimaliseerd op een inter-PON basis. Het onderzoek in dit proefschrift richt zich op de technieken die zijn gebruikt om de bandbreedte-optimalisatie te realiseren, waarbij gebruik is gemaakt van Linear Programming Techniques. Tevens wordt de architectuur beschreven die gerealiseerd kan worden, om een optische service richting de abonnee te kunnen garanderen; daarbij wordt gebruik gemaakt van de conventionele protocollen. Tot slot wordt een vooruitblik gegeven op de controle van het beschreven netwerk.

Rajeev Roy 


\section{Table of Contents}

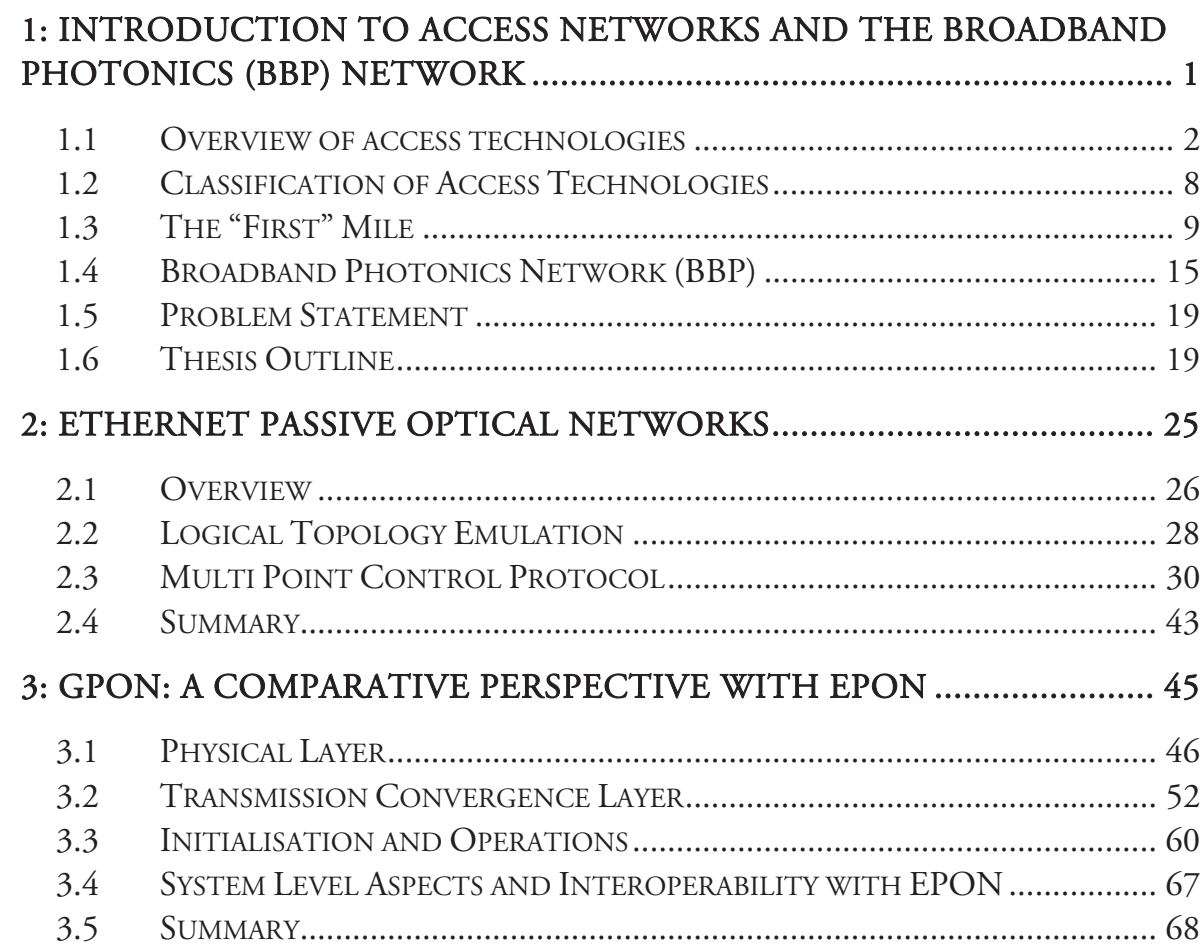

4: CASE FOR DYNAMIC RECONFIGURABILITY IN ACCESS NETWORKS

\begin{tabular}{|c|c|}
\hline 4.1 & CONCEPT OF RECONFIGURABILITY IN AN ACCESS NETWORK .................... 71 \\
\hline 4.2 & DEMAND PROFILING \\
\hline 4.3 & STATIC NETWORK \\
\hline 4.4 & 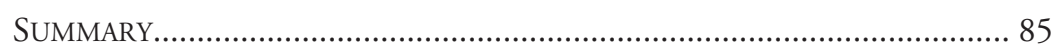 \\
\hline
\end{tabular}

5: BANDWIDTH MANAGEMENT TECHNIQUES …............................... 87

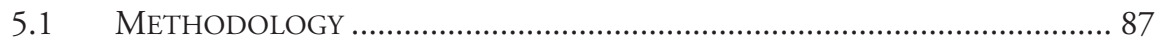

5.2 DEMONSTRATION OF METHODOLOGY ………….................................... 94

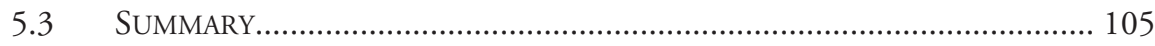

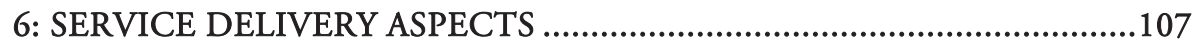

6.1 ETHERnet PerSPective Of THE NeTWORK........................................... 107

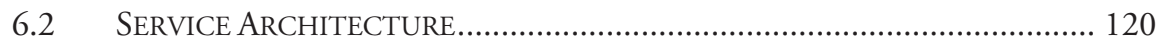

6.3 SOFTWARE AND CONTROL Plane PERSPECTIVE....................................... 131

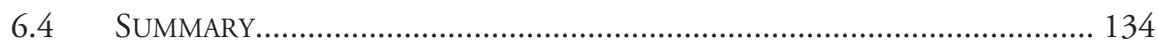




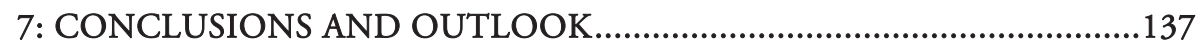

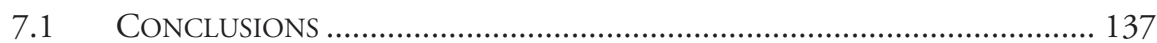

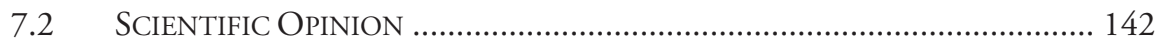

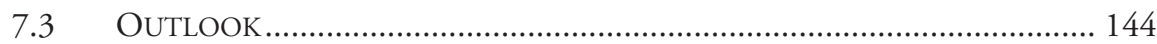

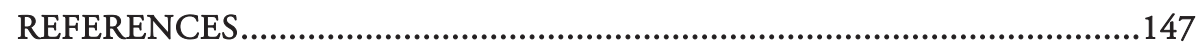

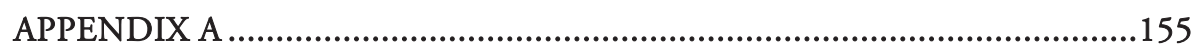

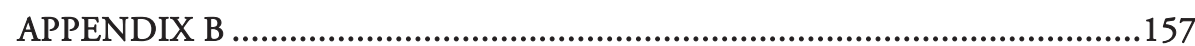

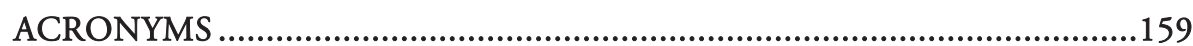

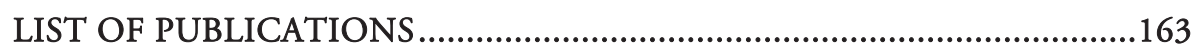

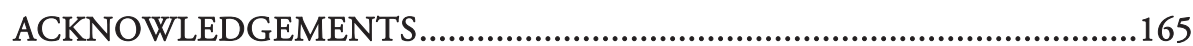

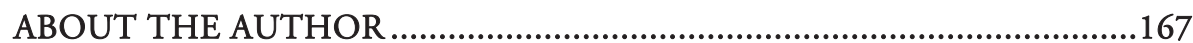




\title{
Chapter 1
}

\section{Introduction to Access Networks and the Broad- band Photonics (BBP) Network}

\begin{abstract}
An Access Network is described as that part of the network which connects the end subscriber to the immediate service provider. Over a period of time this span of network which has traditionally been described as the last mile in networking has received increasing attention and in current times is often referred to as the "first mile" to show the significance of this part of the network. Without a properly dimensioned and designed access network, from an end user's perspective, any technology advances deeper in the network are mitigated. Access networks have shown a metamorphosis in encompassing a multitude of technologies for implementation. The Broadband Photonics (BBP) Network which is introduced in this chapter is one such manifestation of this continual evolution. This concept has been developed under the Freeband consortium of projects and it investigates the design of a dynamically reconfigurable optical access network which can deliver optimal bandwidth services to the end user.
\end{abstract}

Access networks form the critical link in facilitating provisioning of services to an end user. Over a period of time this segment of the network has seen an evolution in the technology used. For wired access networks the medium of transmission- copper or fiber, is just one simple facet of this change. Many different technologies co-exist 
in this segment and the different access technologies should be compared in a multi faceted manner to understand their differences and their similarities. For an end user however, the underlying technology can remain transparent as long as optimal service delivery can be ensured.

\subsection{Overview of access technologies}

The use of the term Access also signified the transition from a traditional "voice only" to a "voice and data" over the network which was essentially designed to support only voice traffic. A theoretical maximum of $56 \mathrm{~kb} / \mathrm{s}$ downstream and $33.6 \mathrm{~kb} / \mathrm{s}$ upstream (upgraded to $48 \mathrm{~kb} / \mathrm{s}$ with enhancements) data transfer rate was possible with the Plain Old Telephone System (POTS) infrastructure [1], [2]. The introduction of the Integrated Services Digital Networks (ISDN) was the first attempt to optimise services not based exclusively on voice [3]. The Basic Rate Interface (BRI) provided up to $128 \mathrm{~kb} / \mathrm{s}$ symmetrical communication over two bearer channels. Use of ISDN also marked the transition from analog to digital transport. This technology also led to the use of the term Broadband for access networks with ITU-T recommendation I.113 defining it as having capability of transmission speeds higher than what is supported by the ISDN Primary Rate Interface (PRI) channels. This would translate to transmission speeds in excess of at least $1.5 \mathrm{Mb} / \mathrm{s}$.

Another aspect of Broadband as defined in industry and government circles is the concept of an "always on" connection unlike dial-up technologies of the past. The ISDN DSL (IDSL) was a precursor to Digital Subscriber Line (DSL) based technologies. Use of the term Broadband still remains synonymous with DSL technologies. The first generation DSL technologies offered a downstream data rate of up to $8 \mathrm{Mb} / \mathrm{s}$. This was increased to up to a $100 \mathrm{Mb} / \mathrm{s}$ in second generation DSL variants. Table 1-1 lists an overview of some of the DSL variants, including the maximum achievable data rates and the loop lengths supported. These variants are collectively referred to as $\mathrm{xDSL}[4]$. 
Table 1-1: Digital Subscriber Loop Variants

\begin{tabular}{lll}
\hline DSL Variant & $\begin{array}{l}\text { Max } \\
\text { Up/Downstream } \\
\text { Data Rate }(\mathrm{Mb} / \mathrm{s})\end{array}$ & $\begin{array}{l}\text { Max } \\
(\mathrm{km})\end{array}$ \\
\hline ISDN DSL (IDSL) & $0.128 / 0.128$ & 5.5 \\
\hline High Data Rate DSL (HDSL) & $1.5 / 1.5$ & 3.7 \\
\hline Symmetric DSL (SDSL) & $2.3 / 2.3$ & 3 \\
\hline Symmetric HDSL (SHDSL) & $4.6 / 4.6$ & 5 \\
\hline Asymmetric DSL (ADSL) & $1 / 10$ & 5.5 \\
\hline Asymmetric DSL2 (ADSL2) & $1 / 12$ & 5.5 \\
\hline Asymmetric DSL2+ (ADSL2+) & $1 / 20$ & 5.5 \\
\hline Very High Bit Rate DSL (VDSL) & $10 / 10$ (Symmetric) & 1.3 \\
\hline Very High Bit Rate DSL (VDSL) & $1.5 / 52$ & (Asymmet- \\
\hline Very High Bit Rate DSL2 (VDSL2) & 1.3 \\
\hline
\end{tabular}

The prior mentioned technologies all use the telephony infrastructure and fall within the operational realm of telecom (telco) operators. In parallel non-telco operators have played an important role in the access with CATV operators being the most noticeable by using non-telco infrastructures. The end user uses a Cable Modem $(\mathrm{CM})$ to communicate with the Headend (HE) over the cable infrastructure, this is either a full copper or a hybrid fiber plus copper ("coax") (HFC) network. Unlike $\mathrm{xDSL}$, instead of being a physical point-to-point connection, a shared medium is used and the effective data rates depend on the number of simultaneous users. Data Over Cable Service Interface (DOCSIS) defines the physical and data link layer specifications for this type of access [5], [6], [7]. 

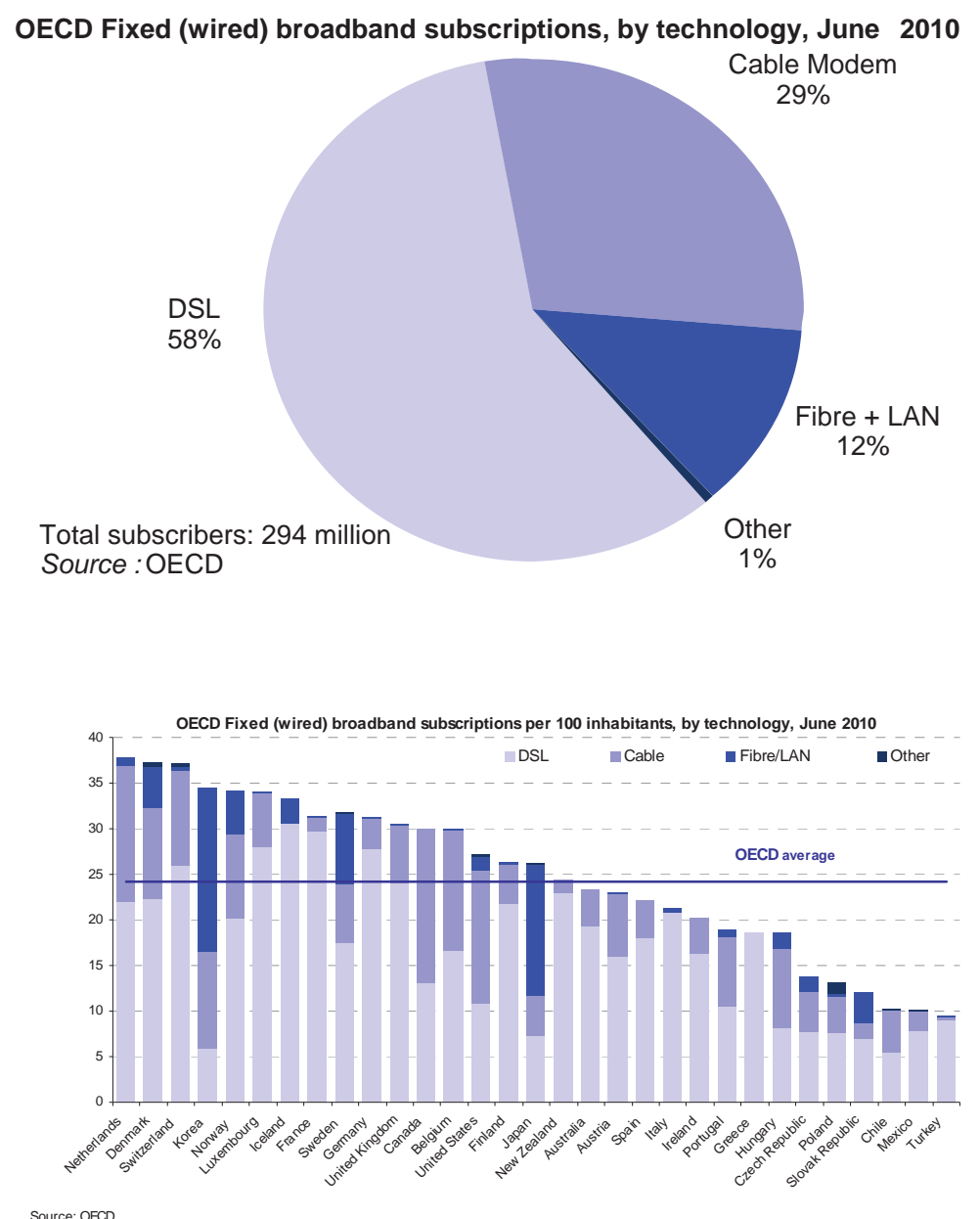

Figure 1-1: OECD data for fixed broadband access

HFCs themselves have evolved to using other technologies for the distribution; instead of using copper for distribution, use of wireless has long been prevalent. This is referred to as the Hybrid Fiber Wireless (HFW) technology. A more recent flavour is to use fiber all through while retaining the DOCSIS technology and the essential HFC architecture. Common reference to such technology is Radio Frequency over 
Glass (RFoG) [8]. Power Line Communications (PLCs) is yet another example where non-telco players are active. Like HFC, use of existing power supply installation is used to provide access. Unlike HFC however, the technology has not seen wide penetration in access. Figure 1-1 illustrates the OECD data (June 2010) for fixed broadband access technology [9], [10]. The pie chart illustrates the shares of the different technologies on a cumulative scale with the bar chart illustrating the break up of technology per 100 inhabitants by country. While DSL and cable technologies show a significant share in deployments, fiber based technologies for fixed access have shown a steady growth in the past years.

Table 1-2: Ethernet for P2P over fiber

\begin{tabular}{|c|c|c|c|c|}
\hline \multicolumn{2}{|c|}{ P2P Ethernet type for Access } & $\operatorname{Max}$ & Optics ${ }^{(1,2)}$ & Max \\
\hline Ethernet & 10BASE-FL & $10 / 10$ & Dual MM, $850 \mathrm{~nm}$ & 1.0 \\
\hline \multirow{3}{*}{$\begin{array}{l}\text { Fast } \\
\text { Ethernet }\end{array}$} & 100BASE-FX & \multirow[t]{3}{*}{$100 / 100$} & Dual MM, 1310 & 2.0 \\
\hline & 100BASE-LX10 & & Dual SM, $1310 \mathrm{~nm}$ & 10.0 \\
\hline & 100BASE-BX10 & & $\begin{array}{l}\text { Single SM, } \\
1310 / 1550 \mathrm{~nm}\end{array}$ & 10.0 \\
\hline Gigabit & 1000BASE-SX & \multirow[t]{5}{*}{$1000 / 1000$} & Dual MM, $850 \mathrm{~nm}$ & 0.55 \\
\hline \multirow[t]{4}{*}{ Ethernet } & 1000BASE-LX & & $\begin{array}{lll}\text { Dual } & \text { MM, } & 1310 \\
\mathrm{~nm} & \end{array}$ & 0.55 \\
\hline & & & Dual SM, $1310 \mathrm{~nm}$ & 5.0 \\
\hline & 1000BASE-LX10 & & Dual SM, $1310 \mathrm{~nm}$ & 10.0 \\
\hline & 1000BASE-BX10 & & $\begin{array}{l}\text { Single } \\
1310 / 1550 \mathrm{~nm}\end{array}$ & 10.0 \\
\hline
\end{tabular}

1: SM: Single mode fiber

2: MM: Multimode fiber 
Development of Passive Optical Network (PON) technologies ushered in the widespread use of fiber in the access. Fiber to the Home/Business/Curb/.. (FTTx) is a buzz word in access parlance and while PONs form a very visible and significant way for implementation, it is not the only way to do so. Active Point-to-point (P2P) connections using Ethernet, often referred to as LAN technologies are also deployed in access using fiber as the physical medium of transport. But just as DSL is synonymous with broadband, FTTx remains synonymous with PON and sees larger deployments than active P2P variants [11]. Typical P2P Ethernet technology became more prevalent with the availability of cheaper optical transceivers and they were simply extensions of LAN connectivity not being limited by reach of transmission over copper or collision domains as in a shared medium.

Table 1-2 lists Ethernet types typically used for fiber access; variants with longer reach are available but not typically used in access. PON technology on the other hand was developed for specific use with fiber. A PON essentially emulates a P2P connectivity over a shared medium. The downstream transmission from the headend to the end user is a broadcast, with the end user discerning on the information intended for it. Unlike a P2P connection however, the upstream communication is a many to one communication over a shared medium and requires some form of arbitration. Current generation commercial PON deployments like the IEEE specified EPON and the ITU-T specified GPON use Time Division Multiplex (TDM) access as a means of arbitration. Following chapters present detailed analysis of these two technologies.

The ever-increasing thirst for bandwidth is now driving the quest for technology development of platforms that can sustain this requirement. Use of dense wavelength division multiplexing (DWDM) technologies have long been established in core and metro networks as a means to increase the aggregate capacity of existing fiber plants. EPON and GPON use WDM only as a means to achieve bidirectional (upstream and downstream) transmission over a single fiber and stop short of using it as a means to increase the aggregate capacity. The use of DWDM in PONs to 
increase the aggregate capacity has seen significant interest in recent times [12]. The increased aggregate capacity of the network can be used for increasing the number of end users, to increase the bandwidth per user, or a judicious combination of both. Recent surveys show the need to have in addition a future-proof infrastructure [13] in access networks. Instead of WDM, increasing the line rates is yet another way PON technologies have matured. The IEEE has standardised symmetric $(10 \mathrm{~Gb} / \mathrm{s}$ upstream and downstream) and asymmetric $(1 \mathrm{~Gb} / \mathrm{s}$ upstream and $10 \mathrm{~Gb} / \mathrm{s}$ downstream) 10GEPON [14]. The ITU-T followed with the standardisation of $10 \mathrm{~Gb} / \mathrm{s}$ variants of GPONs (ITU-T G.987 and G.987x). The $10 \mathrm{~Gb} / \mathrm{s}$ technologies are referred to as the Next Generation PON (NGPON) technologies.

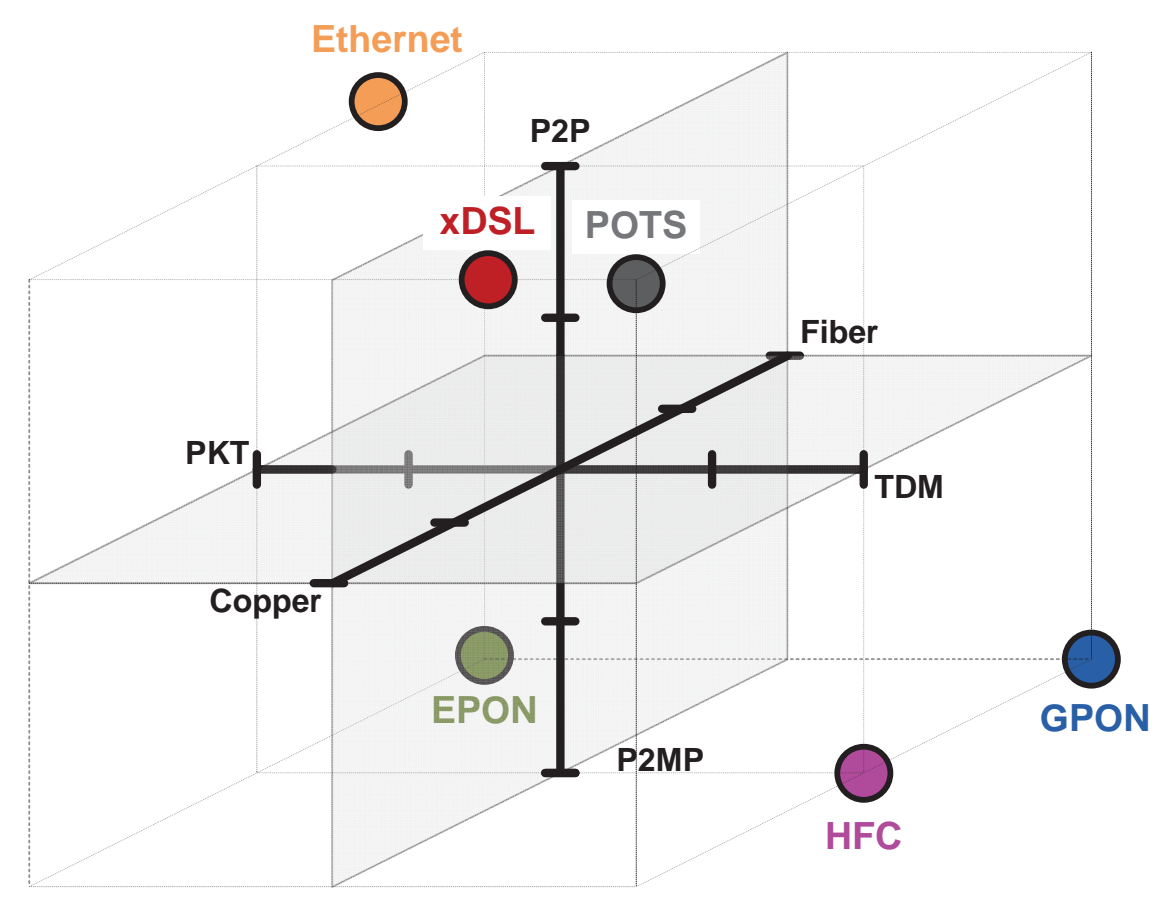

Figure 1-2: Classification of wired access technologies 


\subsection{Classification of Access Technologies}

There are indeed a multitude of available technologies for implementation of access networks. Classification of these technologies helps get a perspective overview of their capabilities and limitations. The question of what parameters to use for the classification is a pertinent one. The easiest and most intuitive is to base the classification in terms of the medium used for it viz. wired or wireless. Traditionally access networks have been wired networks; with the ubiquitous telephone network providing the wired infrastructure required. Wireless access technologies have shown significant developments in recent times and form a whole area of study on their own and are not a focus area here.

Wired access means there is a physical medium connecting to the end user. The specific medium used is another way of differentiating access networks; copper for long has been and yet remains the medium of choice for deployment. Fiber on the other hand has made steady in-roads in the access in recent years. It is not uncommon to find fiber deployments to home however typical deployments in the "first mile" are more commonly a hybrid mix of fiber and copper. Topology is yet another way to view access networks. This is often dependent on the medium of transmission and the technology used for access. The physical topology could be a bus, tree or a ring with the logical topology being a Point-to-Point (P2P) or a Point-toMultipoint (P2MP) over the physical network. Yet another way of viewing the networks would be if the distribution networks are active or passive. An active network has elements which require electrical power for operation in the transmission path. A passive network on the other hand has no powered elements in the transmission path.

Classification of networks with any single yardstick is very restrictive, and any one of the parameters cannot be used as a distinction between all the available access technologies. Figure 1-2 illustrates the position of different technologies presented in a three dimensional plot with the axes representing; TDM or packet (PKT) 
based, Copper or Fiber and P2P or P2MP logical topology. POTS for instance uses copper as a medium of transmission while using TDM access and is a P2P technology, it thus falls on one of the vertices of the cube. xDSL also uses copper as a medium of transmission and is a $\mathrm{P} 2 \mathrm{P}$ technology but is not a strictly TDM technology.

Ethernet which has evolved from use in LAN environments with copper as a medium of transmission has found use in the access networks using a mix of copper and predominantly fiber as a medium of transmission. It retains a P2P topology and is strictly a packet based technology. EPON on the other hand is very similar to Ethernet and is a packet based technology but was designed for operation in a P2MP topology with fiber as a medium of transmission. GPON is also designed for operation with fiber as a medium of transmission with a P2MP topology but unlike EPON, it is an exclusively TDM based technology. HFC uses a mix of copper and fiber as the medium of transmission with a P2MP topology and for data access it is a TDM based technology.

\subsection{The "First" Mile}

Over time, the "First" Mile has seen use of a multitude of technologies. As yet many of them co-exist but the fundamental point which makes them all similar is that this leg of the network connects the end user typically to an aggregation layer which then interfaces to regional and metro area networks (MAN) and eventually to the wide area network (WAN). The typical access customer uses IP based services and Ethernet has come to dominate the home and local area network markets. In this section the connectivity of the end user till the Local Exchange/Central Office, referred to as headend, is presented.

Figure 1-3 illustrates the first mile connectivity over a Public Switched Telephone Network (PSTN) with a dial-up connection and an xDL connection over the same infrastructure. While it is unlikely for an end user to have both a dial-up connection and an $\mathrm{xDSL}$ based connection at the same location, the figure serves the purpose of 
illustrating the difference in data transmission for two cases. For a dial-up modem connection, the IP datagram from a PC is transported by establishing a PPP or HDLC link layer connection to the dial-up modem. This is established typically using a UART physical connection through a serial interface like RS232 or USB. This part of the transmission is digital, the communication between the dial-up modem and a codec in the voice switch at the headend is analog. A V.90 interface is illustrated for the modem as a typical example, however it should be noted that there are a multitude of other modem physical interfaces available. For a dial-up modem the PPP session is peered with a Network Access Server which might be locally present at the headend, or after a PSTN cloud.

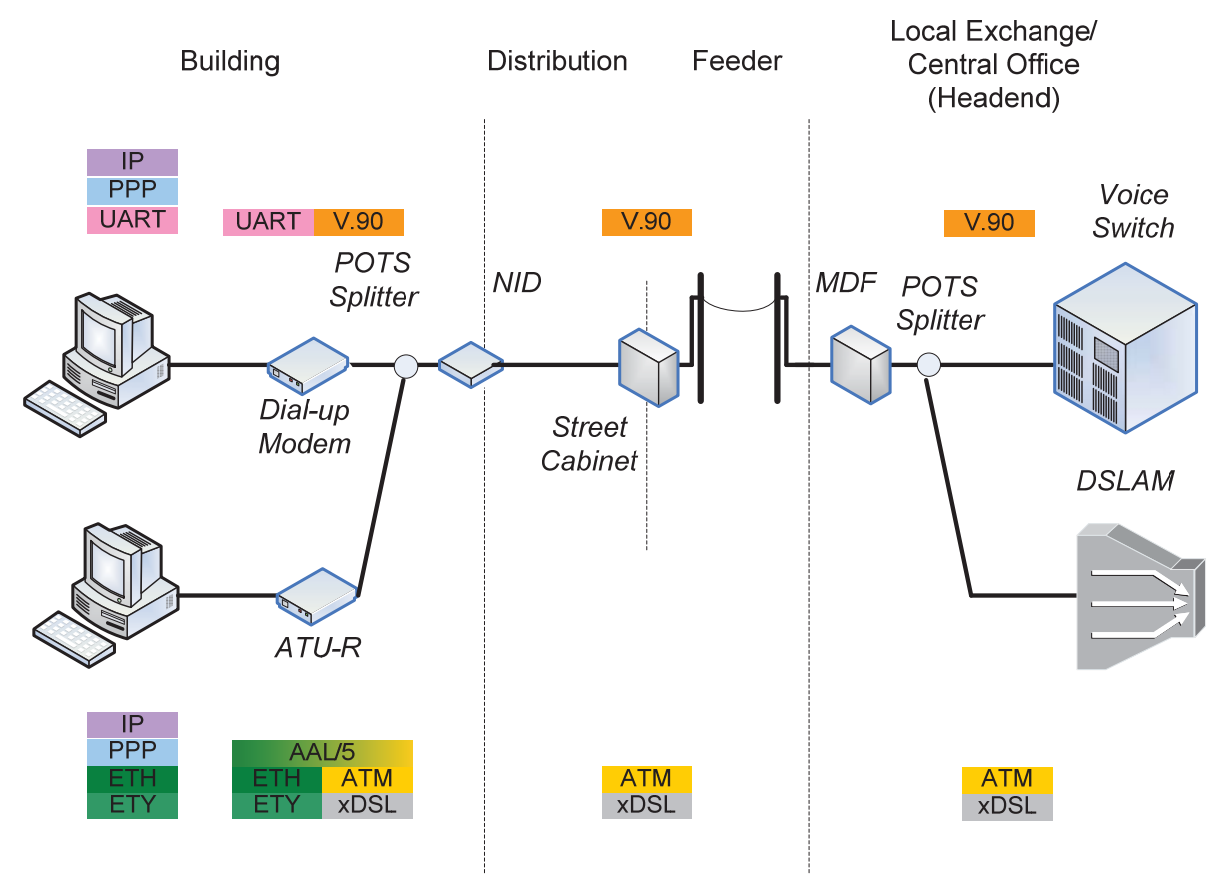

Figure 1-3: Data connectivity over PSTN infrastructure- dial-up modem and xDSL 
An xDSL connection is carried over the same infrastructure and is separated at both ends with a high pass filter. Typical connection of any IP enabled device with the ADSL Termination Unit Remote (ATU-R) is through Ethernet connectivity (ETH/ETY) as illustrated or a WiFi connection. The link layer connectivity is typically still PPP with the IP datagram being mapped over Ethernet for PPPoE (as illustrated) or over ATM directly with PPPoA. The PPP link is peered with a Broadband Access Switch (BAS). Since the data rates possible with DSL are a function of distance, the distance of the distribution section (or loop) is shorter for variants like VDSL. In such implementations the Digital Subscriber Link Access Multiplexer (DSLAM) is located at street cabinets, with uplinks to the headend provided with DS1/E1 connections.

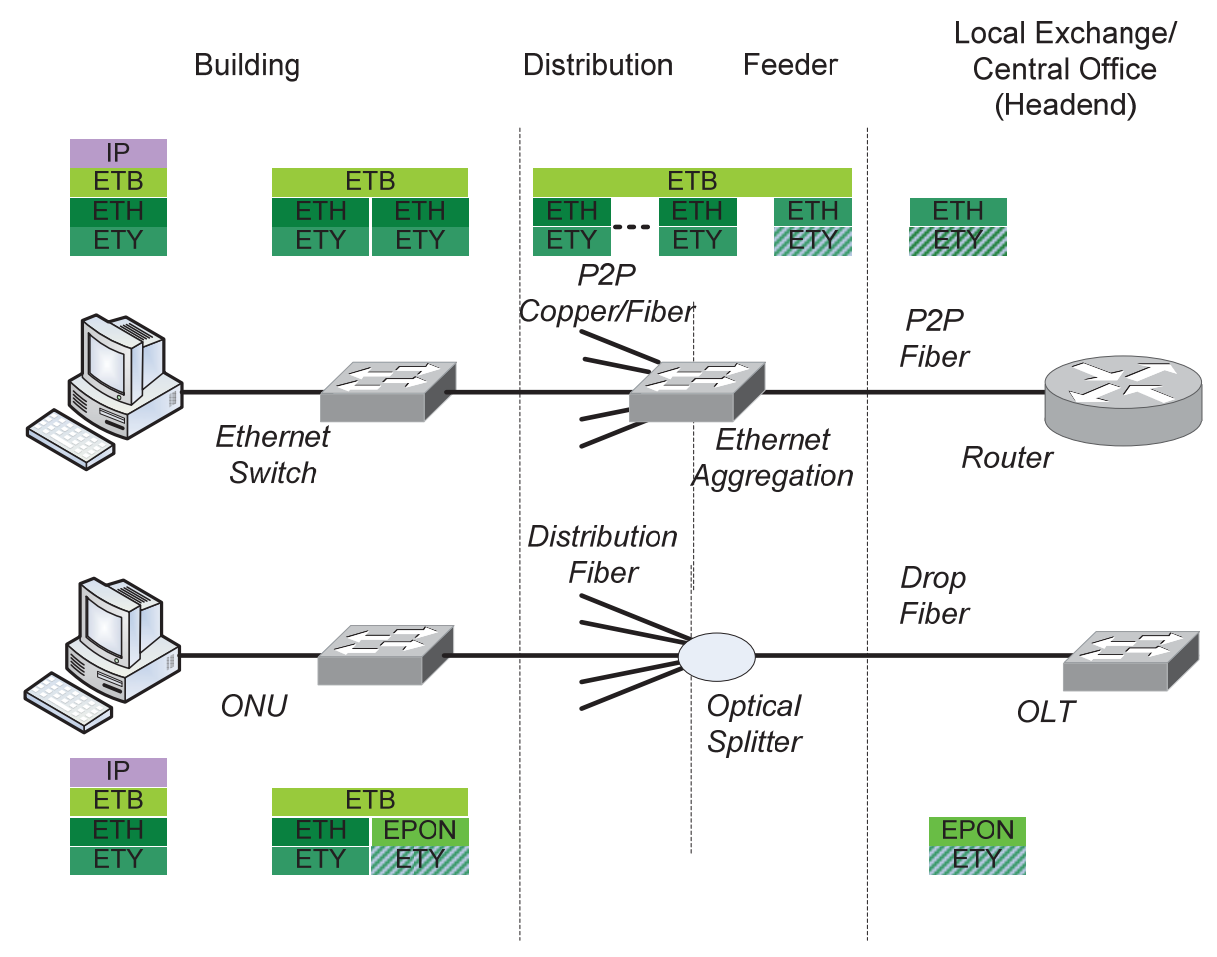

Figure 1-4: Data connectivity -P2P Ethernet and EPON 
Figure 1-4 illustrates an active Ethernet based data connectivity and an EPON system (depicted as FTTH). The street cabinet or remote node location is usually used as an Ethernet aggregation layer, for instance if the end users have point-to-point copper based Fast Ethernet connections, the uplink to the headend could be an optical fiber based Gigabit Ethernet connection. The Ethernet layer can itself be used for link connection and no explicit PPP peering is required, this however depends on operator preference. IP operation blends well with Ethernet and Figure 1-4 also illustrates the use of a bridging/tagging layer (ETB) to enable identification of customer traffic flows.

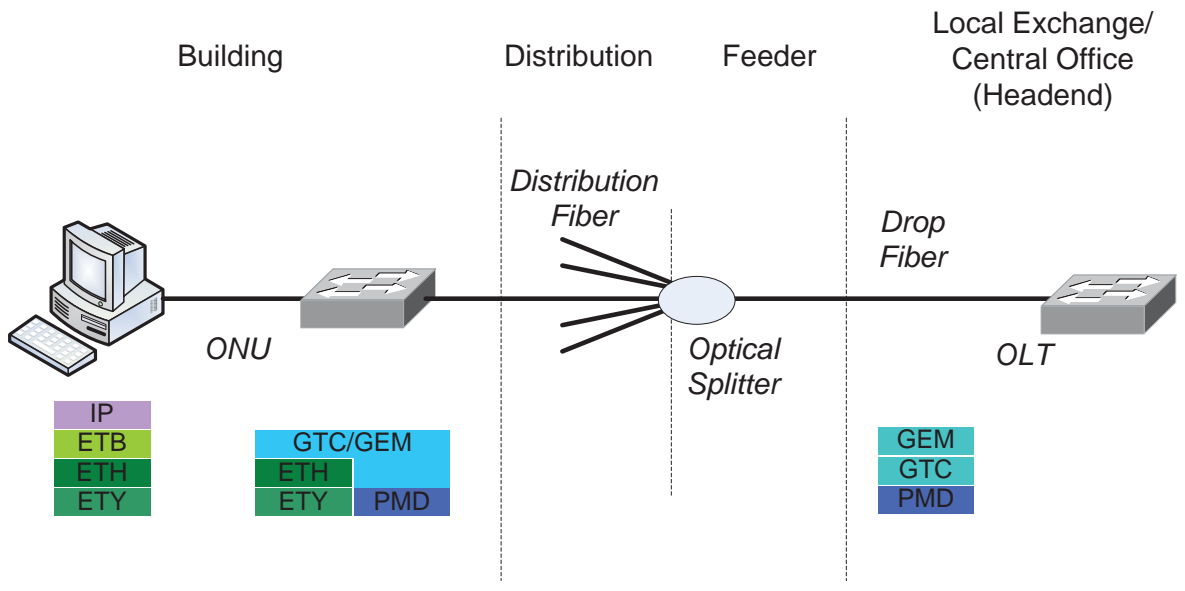

Figure 1-5: Data connectivity- GPON

Ethernet evolved as a technology to allow for simplex transmission over a shared medium without requiring any arbitration. With multiple nodes sharing the same collision domain, the nodes have a system of using a back-off mechanism to randomly delay transmission in case there are collisions. With duplex transmission however the connections are $\mathrm{P} 2 \mathrm{P}$ and thus effectively reducing the collision domain to just a single node without the need for any back-off mechanism. Thus in such connections Ethernet follows the philosophy of always transmitting unless asked not to. EPON operation is very similar to Ethernet, it also emulates a $\mathrm{P} 2 \mathrm{P}$ connection 
but over a P2MP shared medium. Unlike Ethernet, the philosophy of EPON in the upstream is exactly the reverse, of not transmitting unless explicitly asked to. The MAC layer upwards it is exactly same as Ethernet. GPON is a TDM based protocol and uses GPON Transmission Convergence layer (GTC) and the GPON Encapsulation (GEM) method to enable transport of packet based payloads like Ethernet. The GTC provides for mechanisms of establishing link connection and as in Ethernet based systems PPP is not explicitly required. Figure 1-5 illustrates the schematic of data connectivity with GPON.

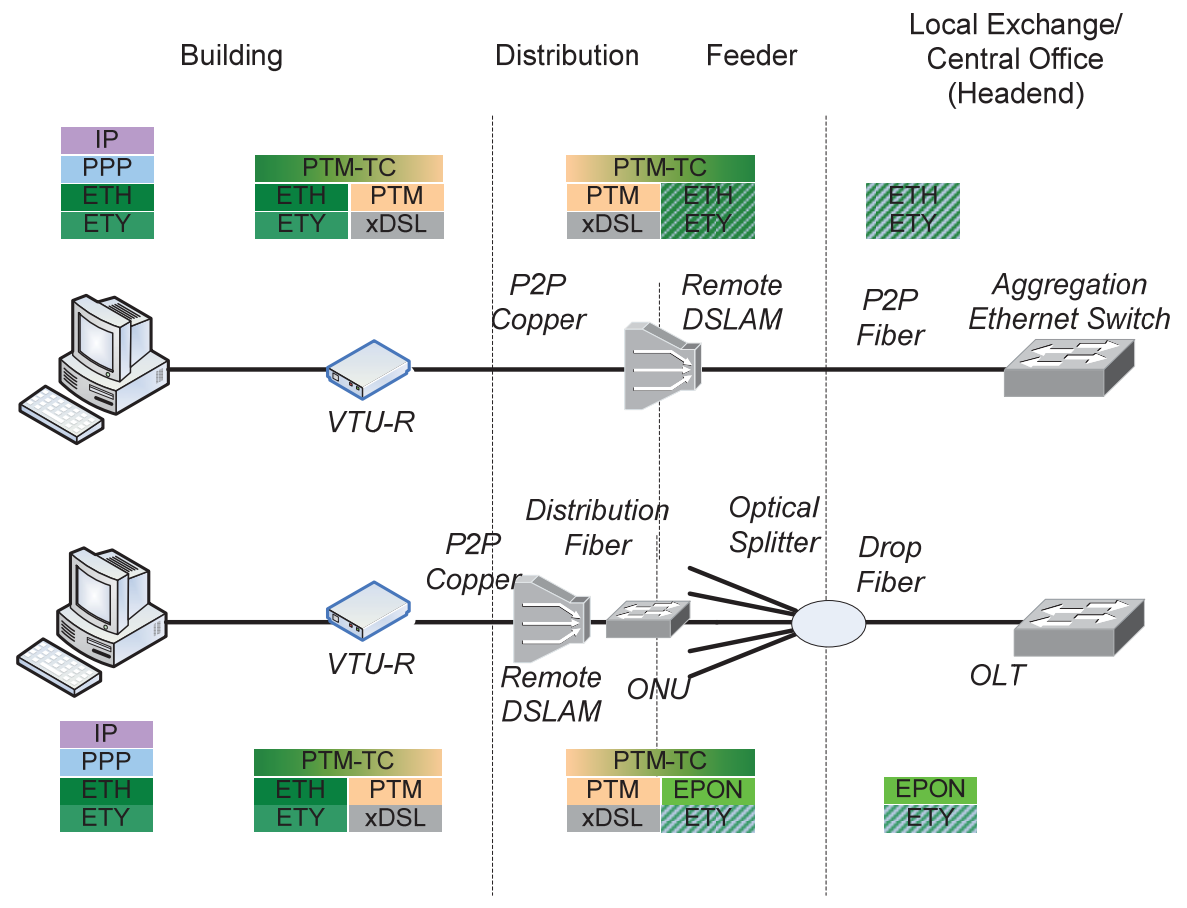

Figure 1-6: Data connectivity- VDSL2 with P2P Ethernet and EPON

A combination of technologies is also common in deployment scenarios. VDSL2 evolution is a prime example where a mix of fiber and copper deployment is seen. Figure 1-6 illustrates connectivity for a VDSL2 based system with the distribution and drop from the street side/building cabinet to the end user is copper based while 
the feeder connectivity is fiber. The fiber connectivity itself can have multiple flavours, in the figure a point-to-point Ethernet connection from the street side/building cabinet to the headend and an EPON connection is illustrated. This also points out the typical Fiber to the Curb/Building (FTTC/B) typical fiber deployment. VDSL2 as a technology offers an alternative to current generation FTTH systems. The use of Packet Transport Mode (PTM) instead of Asynchronous Transport mode (ATM) is illustrated.

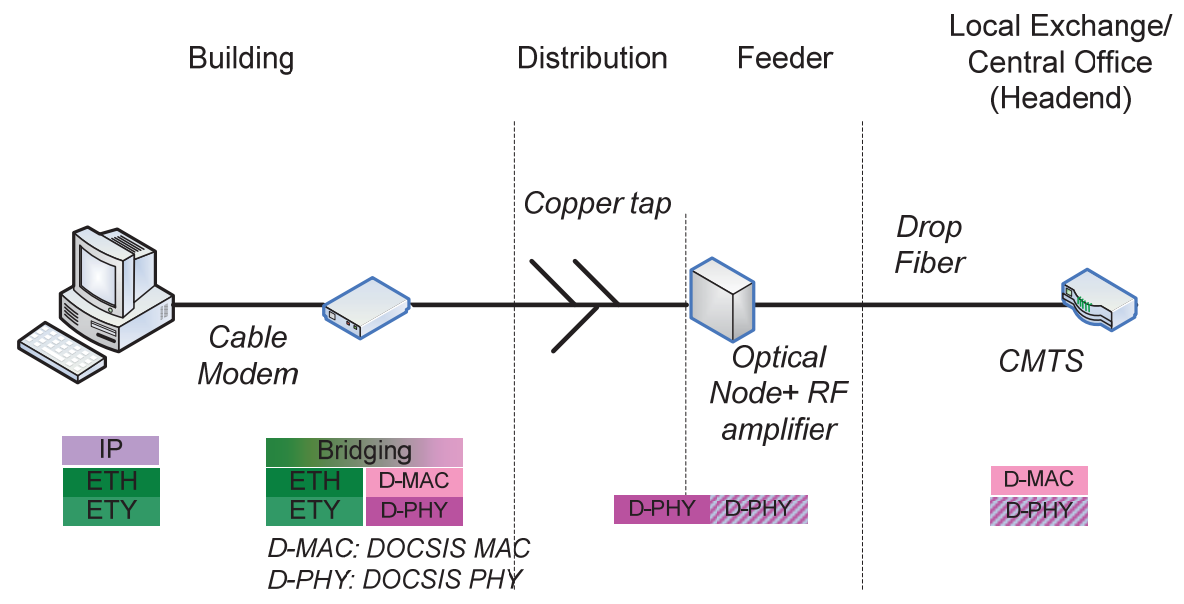

Figure 1-7: Data Connectivity: HFC system

The use of hybrid (Copper plus fiber) connection has been prevalent as HFC over cable distribution networks. Figure 1-7 illustrates the typical set-up for data connectivity over a cable TV network. As in PON systems, the downstream transmission from the headend Cable Modem Termination System (CMTS) is a P2MP broadcast transmission. The upstream likewise is a many to one transmission and a DOCSIS defined TDM+RF arbitration is used to enable connectivity. 


\subsection{Broadband Photonics Network (BBP)}

The trend for increase in bandwidth in access is a reality which network operators have to reckon with. Increase in aggregate capacity supported by a deployed fiber plant can be achieved by either increasing the line rates supported or by supporting multiples of logically separated networks on the same deployment, each operating at a potentially different line rate. CWDM or DWDM technology is the most obvious means to achieve the logical separation in fiber plant deployments. The Broadband Photonics Project (BBP) under the consortium of Freeband projects [15] investigated the design of an optically routed, dynamically reconfigurable access network. It introduces another dimension in the network by facilitating reconfigurability of the network such that the logical configuration of a multi-wavelength network deployment could be changed to optimise bandwidth delivery to the end user and partially mitigate the need to over-deploy resources while allowing for a growth plan in the aggregate capacity of the network. Figure 1-8 illustrates the BBP network schematic [16].

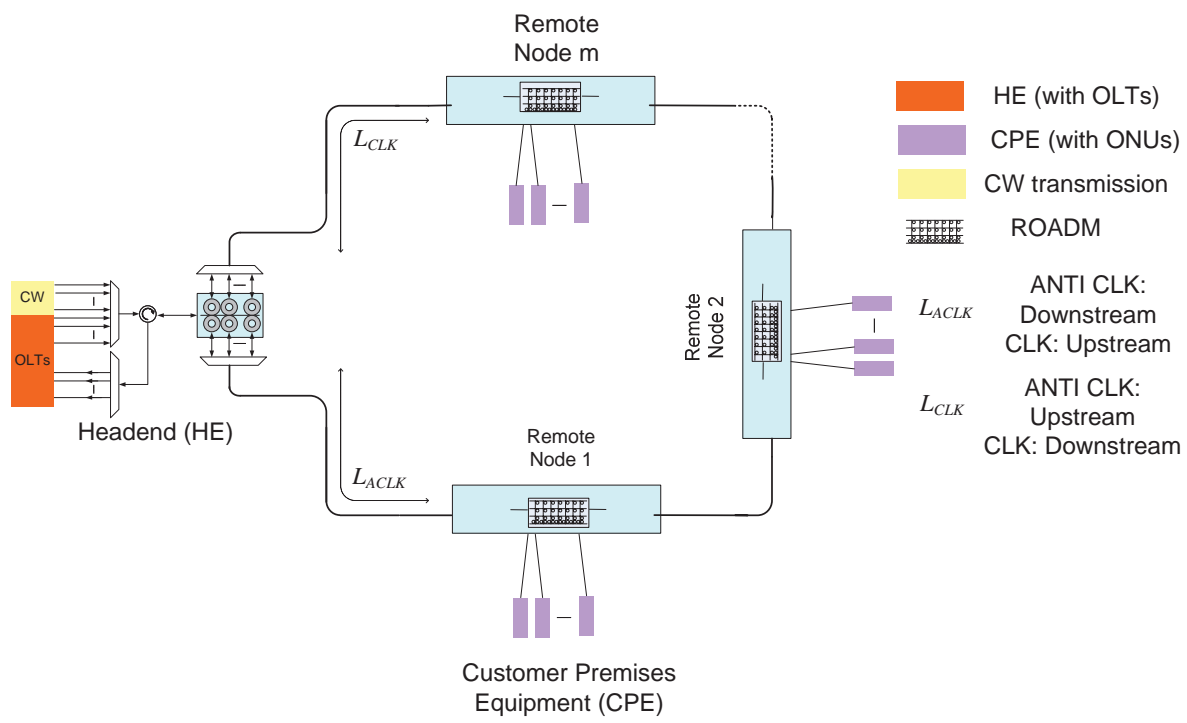

Figure 1-8: BBP network schematic 


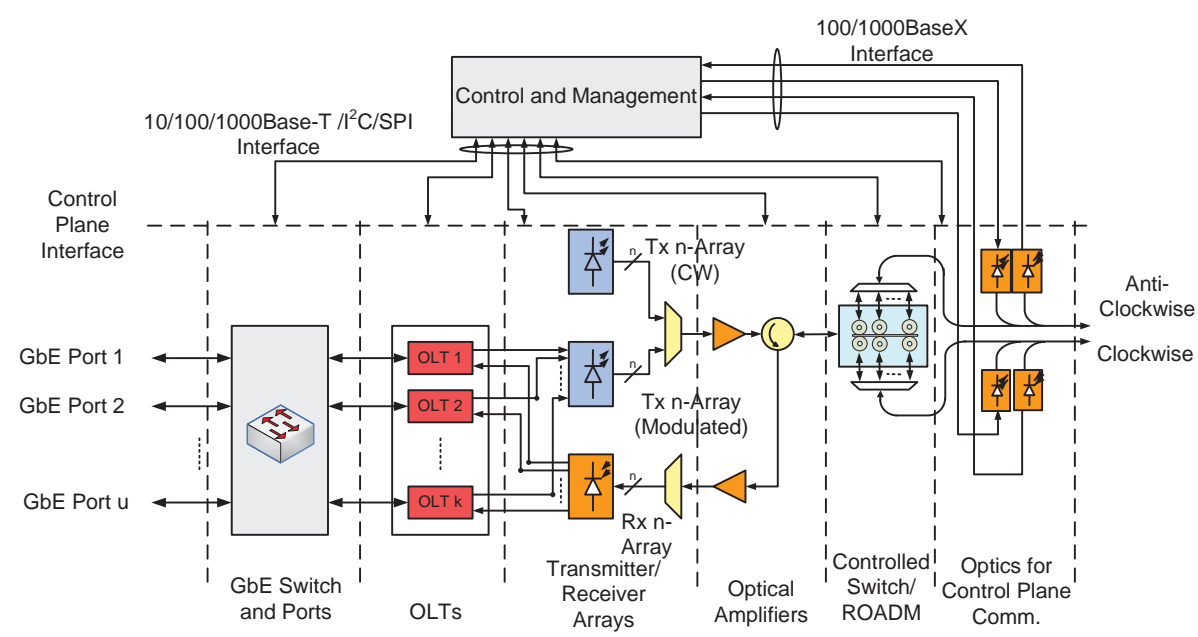

Figure 1-9: BBP Headend schematic

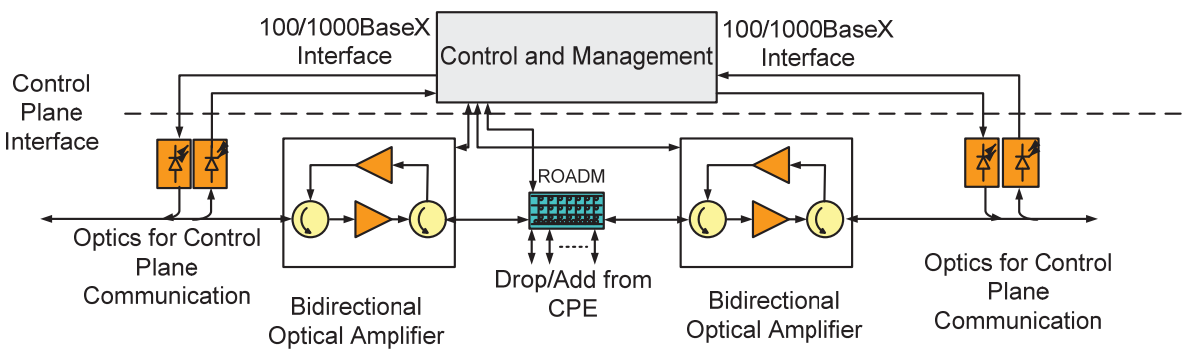

Figure 1-10: BBP Remote Node schematic

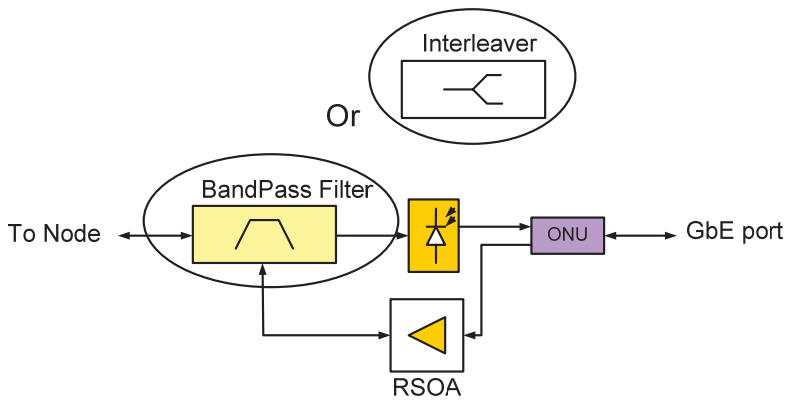

Figure 1-11: BBP Customer Premises Equipment 
A physical ring connects the HE to multiple remote nodes (RNs). Figure 1-9 illustrates the schematic of the HE. The HE location supports multiple OLTs operating on standard PON specifications with modified optics to transmit on the ITU-T 50/100 GHz grid in the $\mathrm{C}$-band. The $\mathrm{HE}$ in addition transmits an equal number of continuous wave (CW) lasers on ITU-T $50 / 100 \mathrm{GHz}$ grid in the C-Band which are modulated at the $\mathrm{CPE}$ and used for upstream communication. Transmission is possible in both clockwise and anticlockwise directions providing redundancy in HERN communication with tolerance up to a single fiber break [17]. The HE also houses the Control and Management (C\&M) for the network with out of band Ethernet based communication with RNs over the same fiber infrastructure [18], [19].

Figure 1-10 illustrates the schematic of an RN. Each RN subtends multiple CPEs. The RN locations house microring-resonator-based reconfigurable optical add/drop multiplexers (ROADMs) that can selectively add/drop wavelength pairs toward any of the CPEs [20]. Figure 1-11 illustrates the schematic of the CPE. The CPEs house an ONU that operates on standard PON specifications with optics modified to receive downstream transmission in the $\mathrm{C}$-band and to use a reflective semiconductor optical amplifier (RSOA) to modulate the received $\mathrm{CW}$ transmission for upstream communication. The ONUs are thus wavelength agnostic and associate with any OLT depending on the wavelength pair add/drop towards it. The wavelength pair on which any one ONU operates is exclusively decided at the HE.

Figure 1-12 illustrates the logical connectivity between the $\mathrm{HE}$ and a diverse set of CPEs. In the figure two such logical PONs are illustrated, the "Red" and the "Blue" logical PONs. The bandwidth per ONU in any one "logical PON" depends on the number of ONUs supported in that network, since the number of ONUs supported in any one such network can be managed by controlling the add/drop wavelength pairs, the bandwidth availability per ONU can be thus controlled. This forms the basic principle of bandwidth management in the network. The scheme also allows for managing the bandwidth distribution on an inter-PON scale rather than manag- 
ing at an intra-PON scale. This allows for use of native PON protocols within the scope of a single logical PON without any modifications.

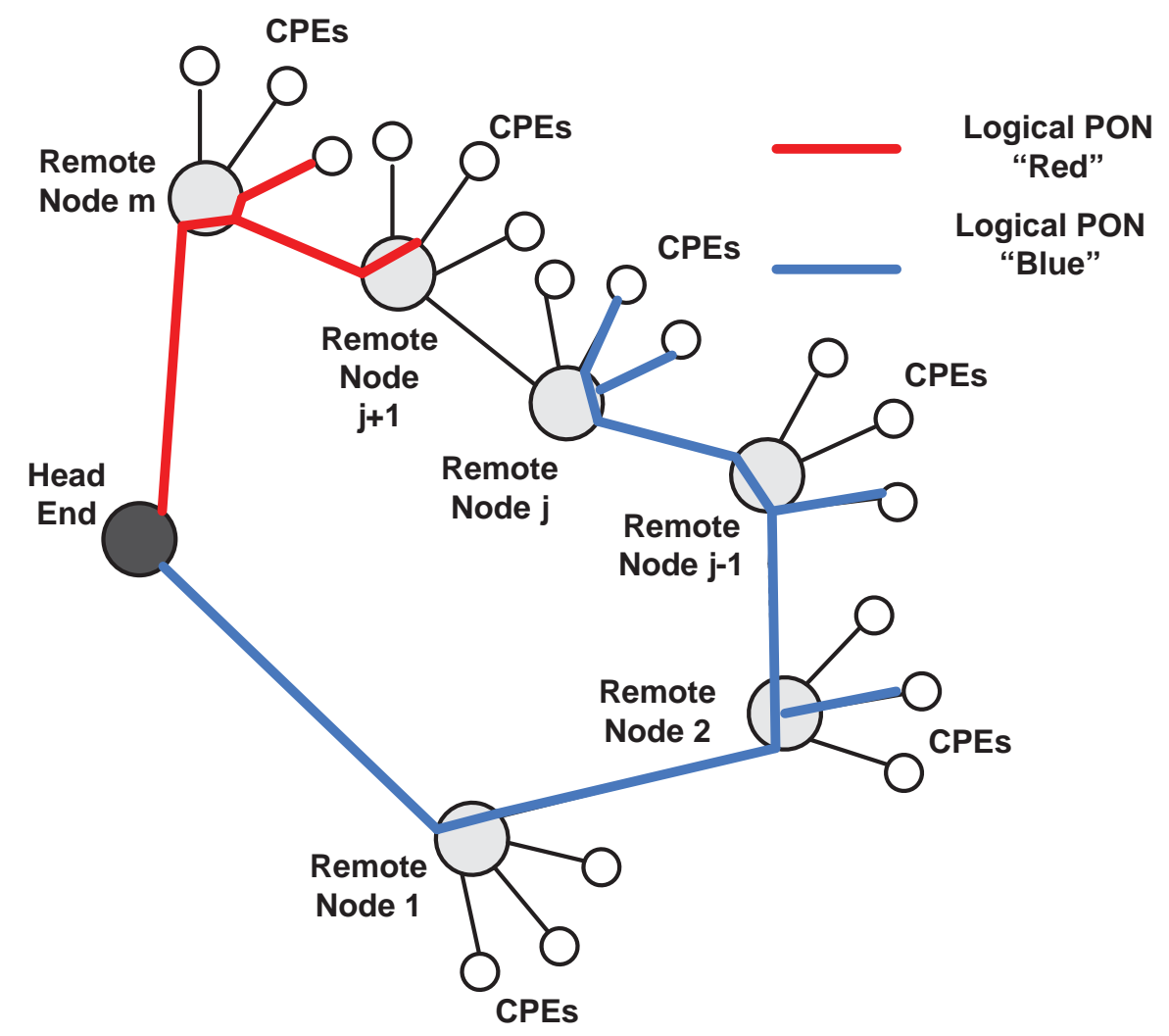

Figure 1-12: Logical connectivity between $\mathrm{HE}$ and CPEs. Two sets of logical PONs are illustrated 


\subsection{Problem Statement}

A physical network provides a platform on which eventually services can be provided to an end user. The physical topology in turn influences the protocols of operation in the network- in particular the data link level protocol. One aspect of the research work was to consider if the evolving (during the course of research) standards in a Passive Optical Network (PON) - the IEEE defined Ethernet Passive Optical Network (EPON) and the ITU-T specified Gigabit-capable Passive Optical Network (GPON) standards could be made operational in a BBP like network. Yet another aspect of the work was to determine if the technologies are inter-operable within the scope of a single network deployment.

In any network, the installed bandwidth capacity can be increased by either installing more network resources or by increasing the data rate of transmission over the existing network. Typically it is a combination of both which is used by network operators. A reconfigurable multi-wavelength BBP like network on the other hand can be seen as a combination of both these approaches along with an added element of reconfiguration. While the use of multiple wavelength pairs allows for an increase in the installed bandwidth capacity of the network (subject to physical limitations), it also provides for a multiple of logically separated network connectivity where different technologies and/or data rates can be deployed. The element of reconfiguration in the network can allow for a more optimal use of the deployed aggregate capacity. The research work had to qualify the motivation of having such a network and thereafter to determine how such a network can be made operational to enable provision of services to an end user.

\subsection{Thesis Outline}

This chapter provides the foundation for the thesis giving an overview of access technologies in general and provides a novel view of classification of access networks with a multi-dimensional perspective. The IP connectivity from an end user to a typical central office location is also presented here. This comprehensive presenta- 
tion is a new contribution of the thesis. An overview of the network architecture using physical components developed within the scope of the project is also presented in the chapter. Definition phase of such network architecture for a reconfigurable network is a new step.

The development of PONs for use in access networks is a relatively new technology. The use of fiber based Point-to-Multipoint (P2MP) topology has necessitated development of bearer (physical and datalink level) protocols. The ITU-T driven Broadband Passive Optical Network (BPON) formed the basis for development of GPON, and the use of traditional Ethernet in the access promoted the development of EPON. Both these technologies were in development during the duration of the research project and since then have been standardised and are commercially the most dominant technologies deployed for use in PONs. Instead of looking at development of yet another bearer protocol for use in the BBP network, one aspect of the research topic was to understand these protocols and consider their use in the network. Chapter 2 provides a detailed qualitative analysis of the EPON technology. The functioning of the protocol is explained. It is analysed how the Ethernet heritage of EPON decides several aspects of EPON operation. However the need to keep the similarity between the two necessitates the use of Ethernet framing with certain differences such that from the Medium Access Control (MAC) layer upwards it is seen as "Ethernet". The analysis of the operational details of the protocol is a new step and forms the basis for understanding how it can be used in a BBP like network

Chapter 3 extends the study to the understanding of GPON. Every aspect of functioning of GPON is compared with equivalent operation in EPON. The understanding the qualitative differences between the two technologies rather than limiting the study to drawing conclusions on bit rate alone is an important aspect. The Chapter clearly shows how the use of GPON based on its development from BPON and its legacy of Synchronous Digital Hierarchy (SDH) relates to frame based operation which is different from the packet based EPON. As in the previous chapter, 
this kind of comparative analysis of EPON and GPON is a new contribution of this work. This chapter also deals with the issue of defining what inter-operability of the two standards means within the context of the BBP network. It is shown that while both technologies are directed at the same segment of the network, they are fundamentally different in the way of operation- one is a packet based technology while the other one is a TDM based technology and they cannot inter-operate in the sense of using an EPON OLT with a GPON ONU and vice-versa. However, since the BBP network uses multiple wavelength pairs supporting "logical" PONs, each such "logical" PON can be independently operated with either EPON or GPON as the bearer protocol.

The unique architecture of the BBP network has been modelled as a stack of logical PONs. Chapter 4 views the possibility of operating native PON protocols in a BBP like network and describes how bandwidth management can be done on a interPON scale rather than looking at an intra-PON environment. While in itself the idea of having a reconfigurable stack of PONs is new, one has to see motivation for deployment of such an infrastructure. Chapter 4 provides a detailed insight into bandwidth requirements in a typical access network using different usage patterns in a typical western European society. It presents the case for a reconfigurable network as the bandwidth requirements of users increase. The aspect of representing the BBP network as a stack of logical PONs which can re-use PON protocols as the bearer protocols without any change in them is a new step. The dimensioning of the bandwidth requirements in such a network is also a new contribution. It is shown that in a geographical area being served by a typical access network there can be large variation in bandwidth demands by the end user. This means that while in some areas there is a higher demand; it is lower in other areas. In a static network deployment it is not possible to practically make use of the unused network capacity from areas where there is less demand. A reconfigurable BBP like network on the other hand can be configured to have a more optimal distribution of bandwidth across the whole network deployment. 
Bandwidth distribution is a key aspect in the whole network deployment. Since the network is reconfigurable with the logical configuration of the network changing temporally, a technique is required which can estimate an optimal network configuration for distribution of bandwidth in the network. Chapter 5 describes this methodology. It shows that the bandwidth can be viewed as a resource which is subject to several constraints based on physical, topological and protocol aspects and shows with use cases that a reconfigurable BBP like network can be used to give optimal bandwidth to the end user. A unique pricing model is described where some users who are ready to sacrifice demand in return for lower tariffs to facilitate resources for additional temporal surges of bandwidth requirement because of an increase in the number of users or an increase in bandwidth demands of existing users. It shows a technique of how a disparity in the network (in terms of bandwidth usage) can be exploited in a reconfigurable network which would otherwise not be possible in a static network deployment. The development of a technique for a reconfigurable BBP like network is a new contribution.

An access network is a platform which connects the end user for facilitation of service delivery. Chapter 6 details the service architecture which can be implemented on such a reconfigurable network. The contribution of looking at the Ethernet aspect of the network is a novel way of depicting the view of the network. The chapter presents the need for service distinction in an access network showing that prioritisation in traffic in terms of class of service helps to maintain a quality of service during periods of congestion. The aspect of depicting that the switch performance during a period of congestion can influence service delivery gives an insight into how the capacity of the switch becomes a factor. This gives new perspective into issues related to jitter generation in different traffic classes even when there is no significant congestion. A framework which can allow defining classes of service has been designed. The definition of the architecture for a BBP like reconfigurable network is a new contribution. Often the concern in a reconfigurable network is that the changing logical topology can be an issue in terms of service delivery. In this chapter a typical user experience for a voice call is depicted to show that while the duration of disrup- 
tion is a concern, the number of disruptions can also become a factor in determining the user experience for a service. This aspect of looking at the number of disruptions rather than just the duration of disruption is a new contribution which is not an obvious question addressed to by network designers. The chapter ends with a detailed perspective view of controlling and managing the network. In any network typically there can be an in and/or out of band control and management channel. While native bearer PON protocols provide the in band channel, the out of band channel is usually an overlay. The software perspective view which can be used for managing the network is presented. These perspective views are important to have an understanding of operating the network and to build software models to manage the network. This is important aspect to enable operation of the network to provision services for an end user.

The thesis ends with Chapter 7 where conclusions drawn from the research work are presented and the outlook for future work is described. 


\section{Chapter 2}

\section{Ethernet Passive Optical Networks (EPON)}

The classic representation of the physical topology of a Passive Optical Network

(PON) deployment is as a tree network. Real world deployments have topologies ranging from the classic tree, to bus, and to ring networks. Irrespective of the actual physical topology, the logical communication flow between the headend and the end customer is always point-to-point. This communication however, needs to be realised in a shared medium. The downstream communication from the headend to the end customer is a broadcast (one-to-many) with the end customer discerning on the information intended for it. Upstream communication from the end customer to the headend is a many-to-one communication and requires some form of arbitration to manage contention. Time division multiplexing (TDM) is one of the ways to prevent contention. The ITU-T specified Gigabit Capable Passive Optical Networks (GPON) and the IEEE specified Ethernet Passive Optical Networks (EPON) are leading standards for commercial deployments of PONs and both use TDM as a means to remove upstream contention. This chapter presents a qualitative description of EPON operations. 


\subsection{Overview}

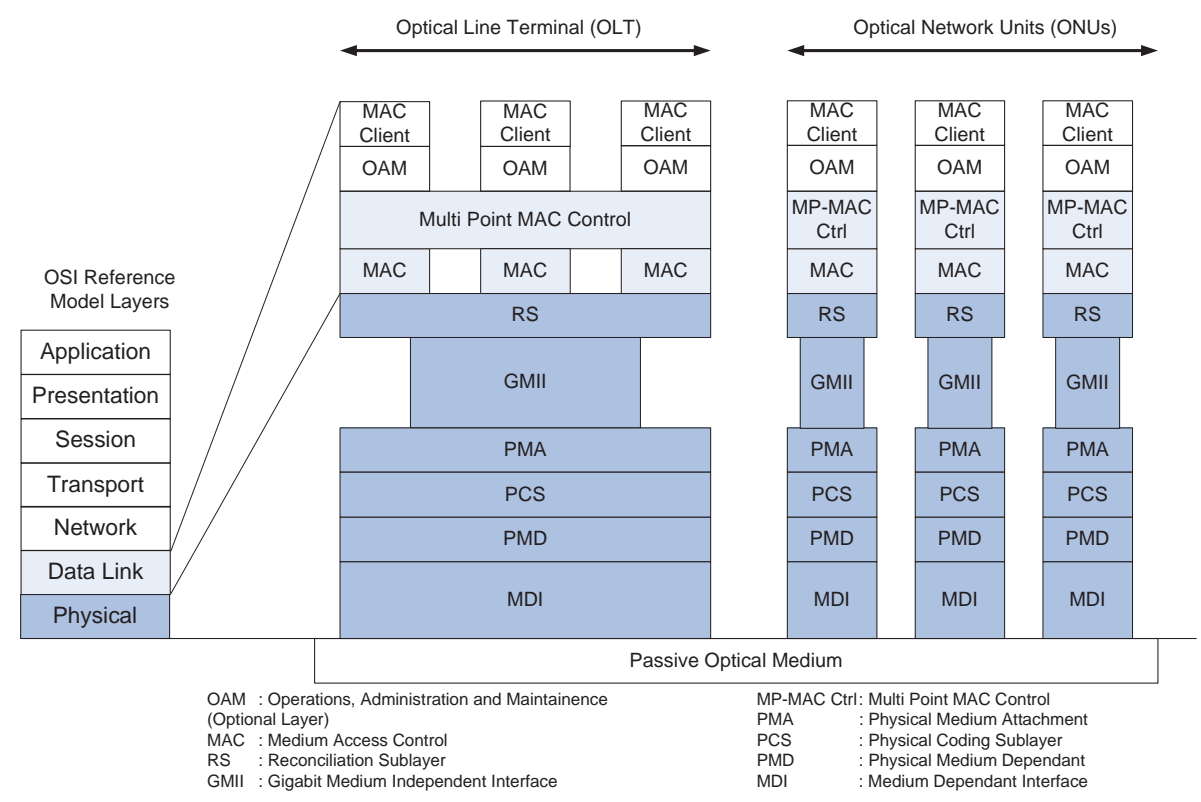

Figure 2-1: Multi Point MAC Control and OSI Stack

The IEEE formed the Ethernet First Mile (EFM) task force in 2001 to extend Ethernet technology to subscriber access areas. As mentioned in the previous chapter, the notation "First Mile" marked the significance shown to this segment of networks. The Ethernet PON (EPON) was standardized with the ratification of the IEEE $802.3 \mathrm{ah}$ recommendations. These have since then been incorporated as sections in the IEEE 802.3-2005 and now the IEEE 802.3-2008 recommendations [21]; the 802.3ah no longer exists as a separate document. The original EPON specification provides for a subscriber access network with symmetric upstream and downstream directions of $1 \mathrm{~Gb} / \mathrm{s}$. In September 2006, the 802.6av task force was formed to investigate extension of EPON operations to $10 \mathrm{~Gb} / \mathrm{s}$. The recommendations of this task force has been ratified and standardised in September 2009 as the IEEE 802.3av-2009 recommendations. This provides for symmetric $10 \mathrm{~Gb} / \mathrm{s}$ upstream and downstream operations and asymmetric $1 \mathrm{~Gb} / \mathrm{s}$ upstream and $10 \mathrm{~Gb} / \mathrm{s}$ 
downstream operations. Figure 2-1 illustrates the EPON protocol stack and compares it against the Open Systems Interconnect (OSI) stack. The specification spans the physical and data link layers.

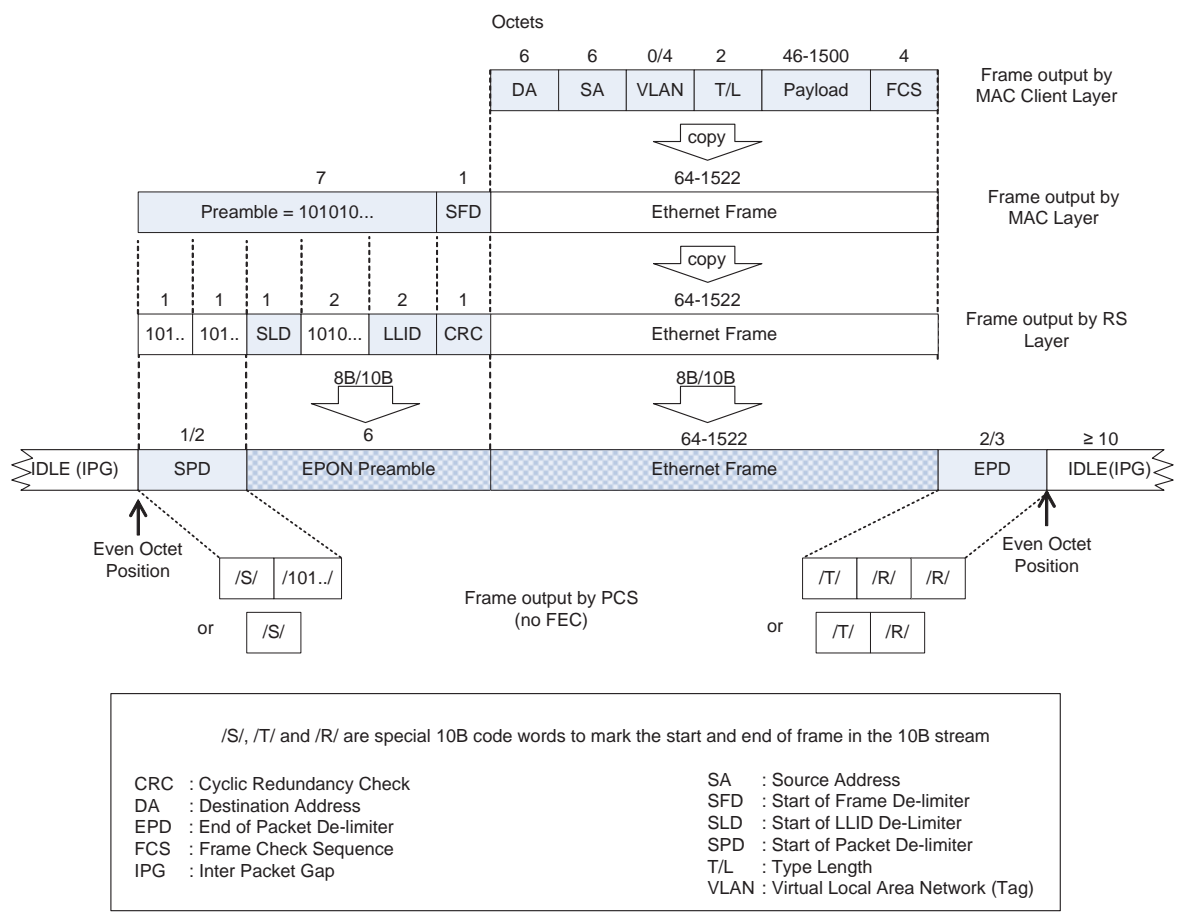

Figure 2-2: EPON frame build up

EPON is a packet based protocol based on Ethernet. Figure 2-2 illustrates the creation of an EPON frame from an Ethernet frame generated by the MAC Client layer. In the early days of Ethernet, the receiving nodes required the preamble to synchronize to individual received frames. The emergence of switched Ethernet networks with duplex transmission where receiving nodes continue to get idle characters, even when no nodes are transmitting to it has made the need for preambles redundant. EPON uses this preamble field to place the Logical Link Identifier (LLID). This identifier uniquely identifies an ONU in both upstream and downstream transmissions. The preamble is inserted by the Reconciliation Sublayer (RS) 
and the use of an LLID within the scope of an EPON network to identify particular ONUs allows for the MAC sublayer to remain unmodified. This is a key point which keeps EPON in essence Ethernet from the MAC layer upwards.

\subsection{Logical Topology Emulation}

Full Duplex switched Ethernet networks are a point-to-point (P2P) topology; EPON tries to emulate this in a shared medium. Figure 2-3 illustrates the logical topology emulation in an EPON. The OLT instantiates as many MACs as is the number of ONUs supported in a network. Each ONU is assigned a unique LLID and the ONU filters frames received on basis of the LLID. The ONU in turn transmits frames with the same LLID, which is subsequently filtered in the RS layer of the OLT and sent to the MAC layer corresponding to respective ONUs. Thus from a MAC perspective the network behaves like a point-to-point Ethernet network. Broadcast in the downstream direction is done through a separate port [21]. ONUs are not allowed to transmit broadcast frames apart from special control frames. Intra ONU communication thus is not possible at the Ethernet/EPON layer and higher layers are required for this. 


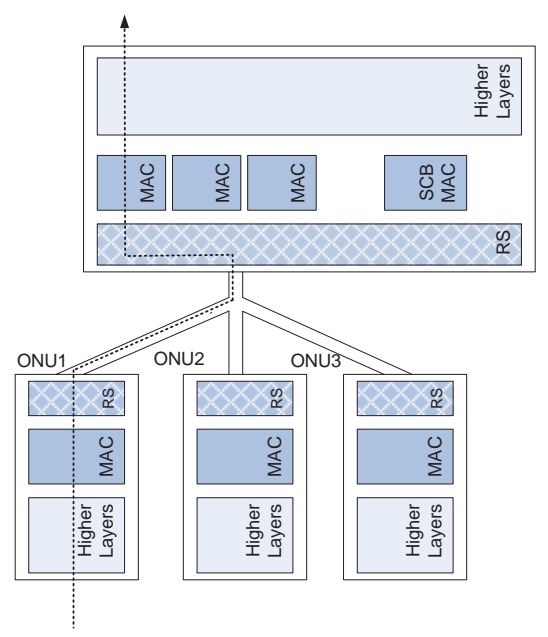

(a) ONU1 communicating with OLT (Upstream Communication)

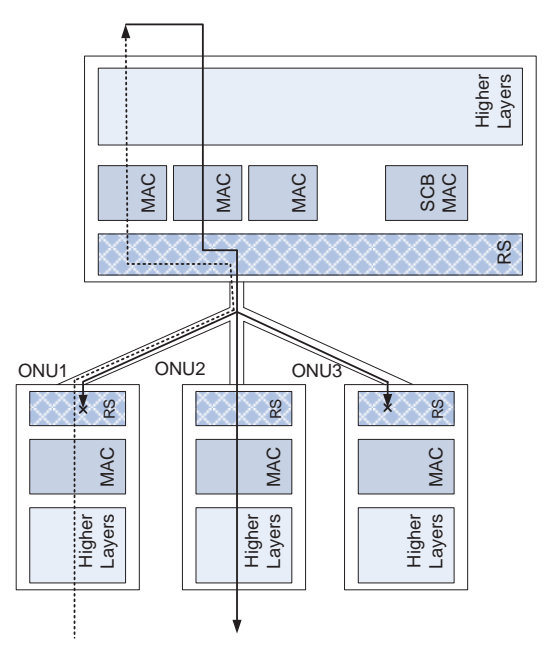

(c) ONU1 communicating with ONU2 (Intra-ONU Communication)

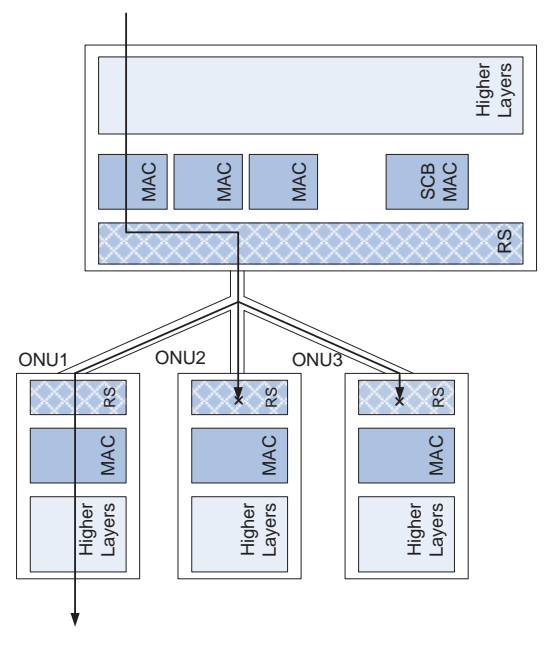

(b) OLT Communicating with ONU1 (Downstream Communication)

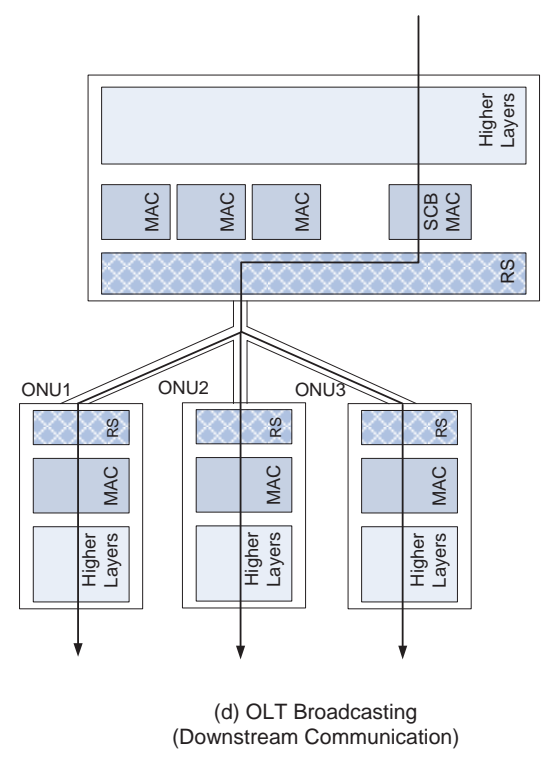

SCB: Single Copy Broadcast

Figure 2-3: Logical Topology Emulation 


\subsection{Multi Point Control Protocol}

PON operations can be categorized very broadly in two steps, the first being initialisation and the second being regular operational state.

The initialisation phase typically consists of Discovery and Ranging: In the Discovery phase the OLT at the head-end tries to discover which ONUs are connected in the network. In the Ranging phase the OLT tries to determine the distance to the ONU so that all nodes are synchronised to the same clock. Networks usually would have Discovery and Ranging at regular intervals to allow newer ONUs to join the network, however this is not a mandatory step and can be an operator initiated phase.

\begin{tabular}{|c|c|c|c|c|c|c|}
\hline 6 & 6 & 2 & 2 & 4 & 40 & 4 \\
\hline DA & SA & $\mathrm{L} / \mathrm{T}$ & Opcode & TS & Field/Pad & FCS \\
\hline
\end{tabular}

DA: Destination Address - MAC control multicast address or that of individual ONUs to which it is addressed

SA: Source Address - Source MAC address of MAC port transmitting or unicast address of the OLT

$\mathrm{L} / \mathrm{T}$ : Length/ Type $(\mathrm{L} / \mathrm{T})-$ Set to $0 \times 8808$ to indicate MAC control frame

Opcode: Specific MPCPDU being transmitted

TS: Time Stamp - Value of 32 bit local time register

Field/Pad: Specific data transmitted by MPCPDU. If not used they are filled with zeros

FCS: Frame Check Sequence - Inserted by underlying MAC instance

Figure 2-4: MAC Control Frame 
In the Operational state the OLT transmits in the downstream direction while the ONUs transmit in the upstream direction. ONUs are allocated bandwidth by the OLT to do upstream transmission. Based on ONU demands the bandwidth allocation is either dynamic or static, this depends on implementations adopted by the network operator. There is always a minimum keep alive indication from the ONU to the OLT even if there is no data transmission required from it.

The MAC Control layer is defined to provide for real-time control and manipulation of $M A C$ sublayer operation [22]. This layer is defined as an optional layer for Ethernet operations and the initial purpose was to define processing of PAUSE frames alone. The scope of this layer has been extended to include definition of additional control frames which are needed to formalise the Multipoint MAC control protocol to facilitate EPON operation. The MAC Control layer thus is a mandatory requirement for EPON operations. A generic MAC control frame is illustrated in Figure 2-4. MAC control frames are of fixed size with 60 octets with 4 octets of FCS added by the underlying MAC layer [22].

Multipoint MAC control frames (opcode) are listed below:

GATE $(0 \times 0002)$

REPORT $(0 \times 0003)$

REGISTER_REQ $(0 \times$ 0004)

REGISTER $(0 \times 0005)$

REGISTER_ACK (0 x 0006)

These messages are formally referred to as the Multi-Point Control Protocol Data Units (MPCPDUs). 


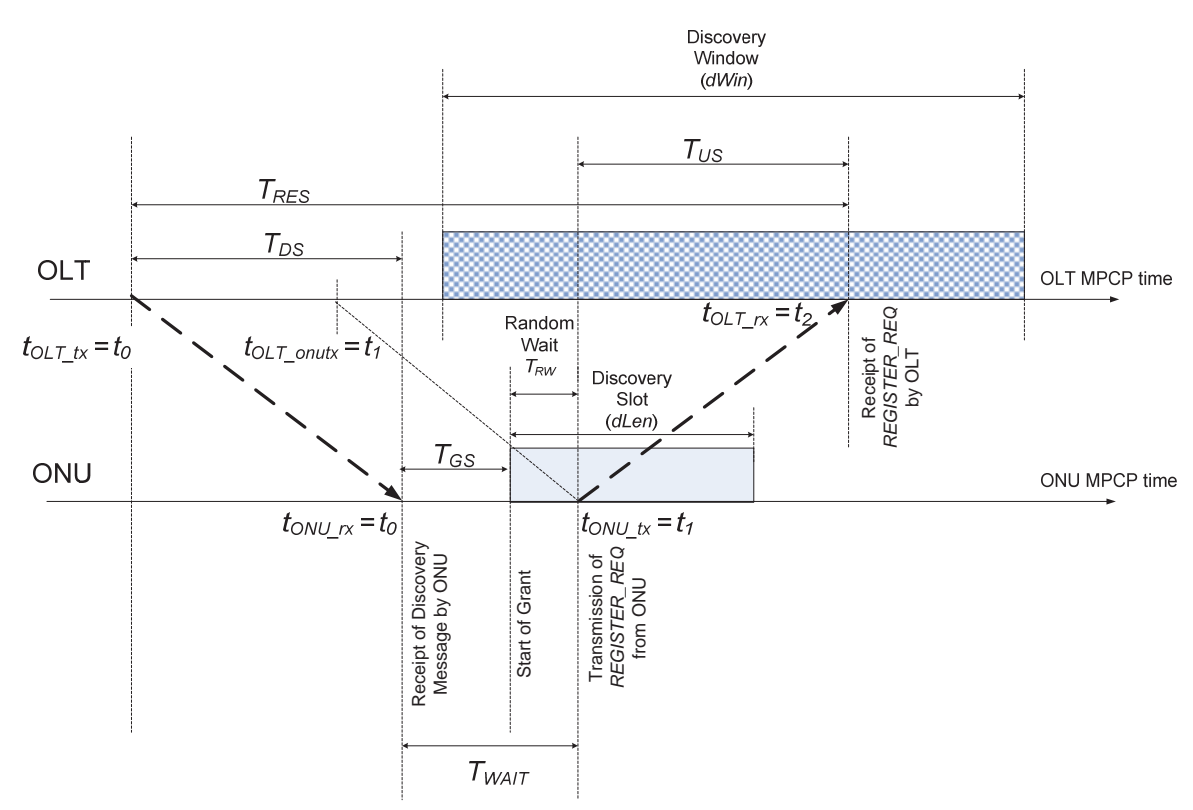

Figure 2-5: Discovery Process

\subsubsection{DISCOVERY AND RANGING}

An OLT initiates the Discovery process with the discovery GATE control frame. In an EPON un-registered ONUs cannot transmit or turn on the laser. These uninitialized ONUs have to wait for an indication from the OLT to start transmission. A discovery round allows for such ONUs to advertise for presence and try to get registered with the OLT. Figure 2-5 illustrates a discovery GATE control message. This is a broadcast message from the OLT to all ONUs.

Once an uninitialized ONU receives such a control message it sets its clock to match the timestamp indicated on the message. The ONU will then wait till the indicated time at which it is allowed to start transmission and then for an additional random delay subsequent to which it advertises its presence with a REGISTER_REQ control message. The duration for which the ONU can transmit is indicated by the length of the grant in the discovery GATE (dLen). The entire time window during 
which the OLT is receptive to any transmissions from ONUs for discovery is called the Discovery Window (dWin). The earliest response an OLT can expect after transmission of the discovery GATE would be from the nearest ONU which starts its upstream transmission without any random wait period. Thus the OLT has to open up the Discovery Window at least at $R T T_{\min }$ after the transmission of the discovery GATE. The window has to be open long enough for the farthest ONU to respond which waits for the maximum possible random delay before upstream transmission. Thus the last transmission the OLT can expect would be at most $R T T_{\max }$ plus $d L e n$ after the transmission of the discovery GATE. Thus there is a minimum required Discovery Window (dWin) as stated in (1). The $d$ Len itself has to be long enough to allow the ONU to transmit at least one REGISTER_REQ message back to the OLT. If there is more time granted the ONU can continue to transmit to the OLT.

$d$ Win $\geq d$ Len $+\left(R T T_{\max }-R T T_{\min }\right)$

Ranging is the process of determining the distance of the ONU from the OLT. All control messages from the OLT including the discovery GATE have a timestamp. On receipt of such a control message every ONU copies the timestamp in the received message and sets its own clock equal to it. All messages sent by any ONU to the OLT contain a timestamp according to the ONU clock (which is a delayed reference of the OLT clock) and thus the OLT is able to determine the round trip delay to every ONU. During a discovery round the control messages sent back by the ONU also allow the OLT to learn the MAC address of every ONU.

$$
\begin{aligned}
& T_{\text {WAIT }}=T_{G S}+T_{R W} \\
& R T T=T_{D S}+T_{U S}=T_{R E S}-T_{\text {WAIT }}=\left(t_{\text {OLT_ } r x}-t_{O_{\text {OLT_t }}}\right)-\left(t_{\text {OLT_onutx }}-t_{\text {OLT_tx }}\right) \\
& R T T=\left(t_{2}-t_{1}\right)
\end{aligned}
$$

Where all points in time are as per the OLT MPCP time axis. 
$T_{\text {WAIT }} \quad$ Time between receipt of a discovery GATE and transmission of a REGISTER_REQ by an ONU

$T_{G S} \quad$ Time indicated for start of grant

$T_{R W} \quad$ Random wait time before ONU transmits a REGISTER_REQ

RTT Round Trip Time. The time taken for a message to transmit from an OLT to an ONU and back to the OLT without any other delays.

$T_{D S} \quad$ Time for message to propagate downstream

$T_{U S} \quad$ Time for message to propagate upstream

$T_{\text {RES }} \quad$ Response time measured at OLT from discovery GATE transmission to REGISTER_REQ receipt from an ONU

$t_{i}(i=0,1 .$.$) \quad Time counter value used at OLT and ONU (Note: ONU$ time counter is a time shifted reference of the OLT time counter)

The time counter at the OLT and ONU is implemented via a 32 bit counter with a granularity of 16 ns called Time Quanta (TQ). The time reference point is with respect to the transmission of the first octet of the MPCPDU. On the receipt of a valid REGISTER_REQ message from an ONU, it is the prerogative of the OLT to register the ONU for operation in the PON. The OLT responds with a unicast REGISTER message which contains a unique LLID for the concerned ONU. This creates a unicast logical link between the OLT and the ONU. During Discovery, the REGISTER message is the only MPCPDU from the OLT which is a unicast message. 


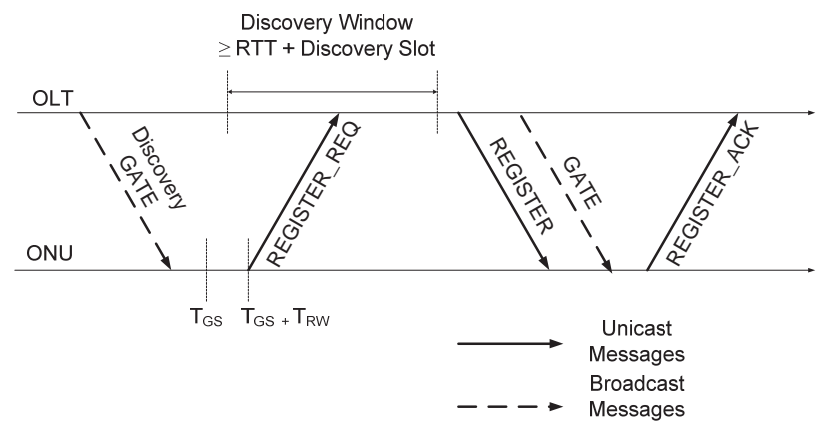

Figure 2-6: Handshake between OLT and ONU during Discovery

The ONU has to respond with a REGISTER_ACK message with which it indicates the completion of the registration process. However to be able to transmit this message the ONU has to receive at least a single grant during which it can transmit this message. This is the first MPCPDU which is transmitted by an ONU to the OLT on the established logical link between them. Unless the REGISTER_ACK message is the first message received, the OLT de-registers the ONU. Figure 2-6 illustrates the handshaking process between an OLT and ONU to establish a logical link.

\subsubsection{OPERATIONAL STATE}

Once the logical link between the OLT and ONU is formed, data transmission can take place. Unlike regular Ethernet where a node can continually transmit unless explicitly asked or forced to stop transmission, in EPON an ONU cannot transmit unless it is explicitly asked to do so. The GATE message is used by the OLT to grant transmission opportunity to an ONU. This control message is technically the same (same opcode) as the discovery GATE message described earlier. However the regular GATE (hereafter referred to as GATE) is not a broadcast message and is directed towards the intended ONU and has a unicast LLID. A bit field indication differentiates the discovery and regular GATE. In addition a discovery GATE does not carry any grants apart from that specifying the allowed time during which an 
ONU can transmit for discovery. A regular GATE can carry up to 4 grants in a single message. Figure 2-7 illustrates the two types of GATE messages.

Grants give an opportunity for the ONU to transmit data. These are allocated in units of TQs and include additional time required for the ONU laser to switch on, OLT receiver to synchronize and for the ONU laser to switch off. A single grant value is stored as 16 bits and thus can be as large as 65536 TQs ( $1.05 \mathrm{~ms})$.

\begin{tabular}{|c|}
\hline Destination Address (DA): 6 bytes \\
\hline Source Address (SA): 6 bytes \\
\hline Length/Type $=0 \times 88-08: 2$ bytes \\
\hline Opcode=0x00-02: 2 bytes \\
\hline Timestamp: 4 bytes \\
\hline Number of grants/flags= 0x09: 1 byte \\
\hline Grant Start Time: 4 byte \\
\hline Grant Length: 2 bytes \\
\hline Sync Time: 2 bytes \\
\hline Pad=0: 31 bytes \\
\hline Frame Check Sequence 4 bytes \\
\hline Bits within frame transmitted \\
left to right (LSB to MSB)
\end{tabular}

(a)

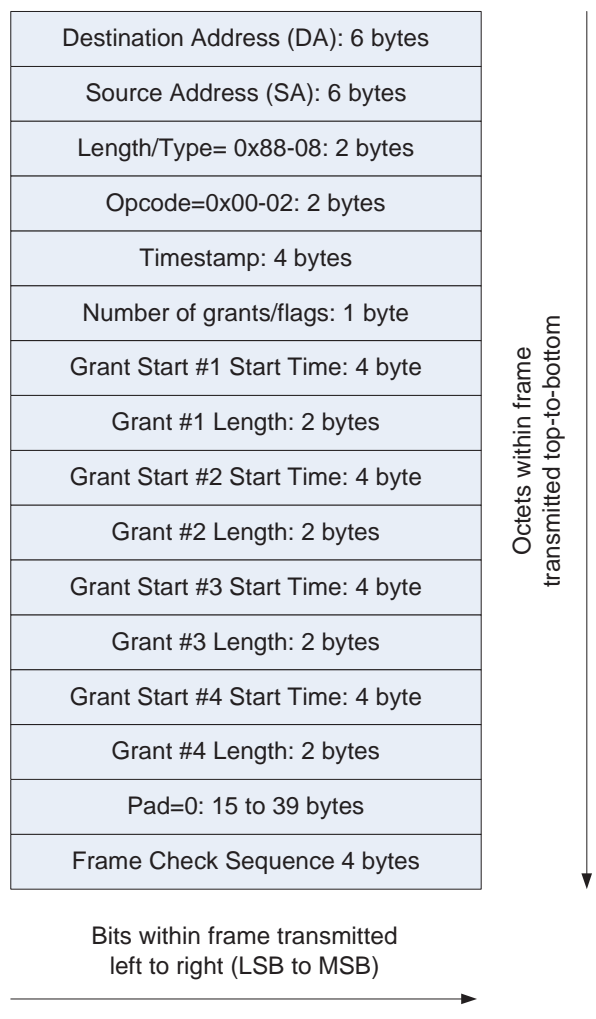

(b)

Figure 2-7: (a) Discovery GATE and (b) regular GATE 
An ONU seeks opportunity for transmission via the REPORT message. Figure 2-8 illustrates a REPORT message. These messages consist of multiple queue sets, each queue set can consist of up to 8 queue reports. The number of queue reports in each queue set follows from the priority requirements of the IEEE 802.1Q[23]. Multiple queue sets are allowed to let ONUs report bandwidth requirements for different thresholds. The number of thresholds that can be defined in a single REPORT depend on the number of queue reports defined per queue set and is illustrated in Figure 2-9.

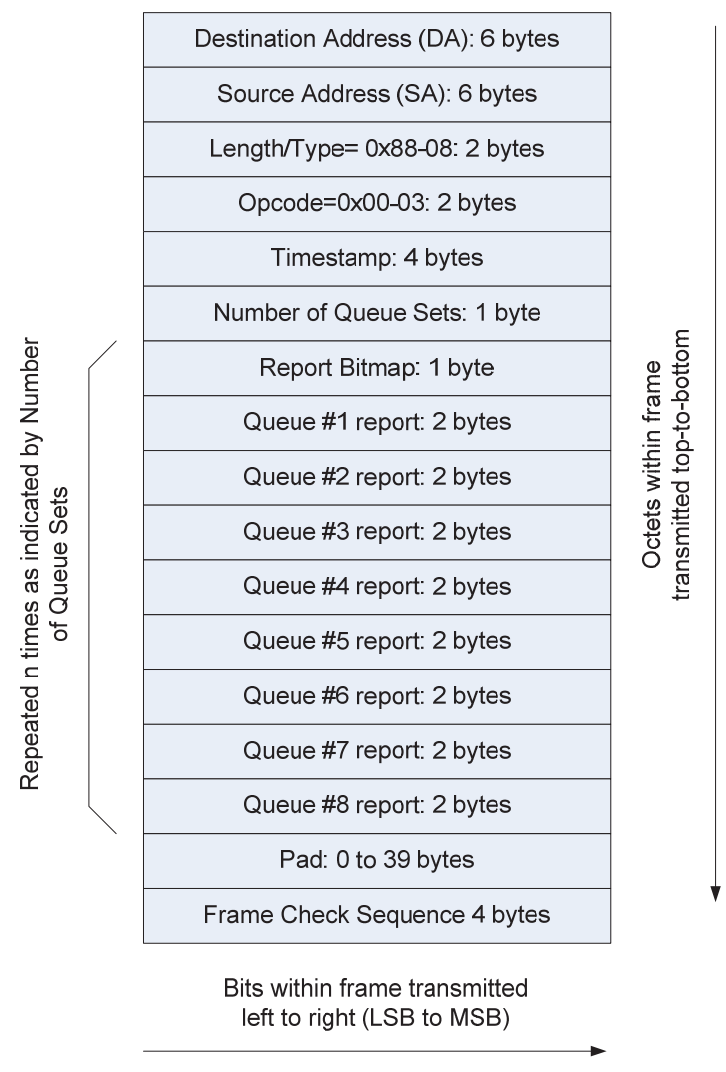

Figure 2-8: REPORT 


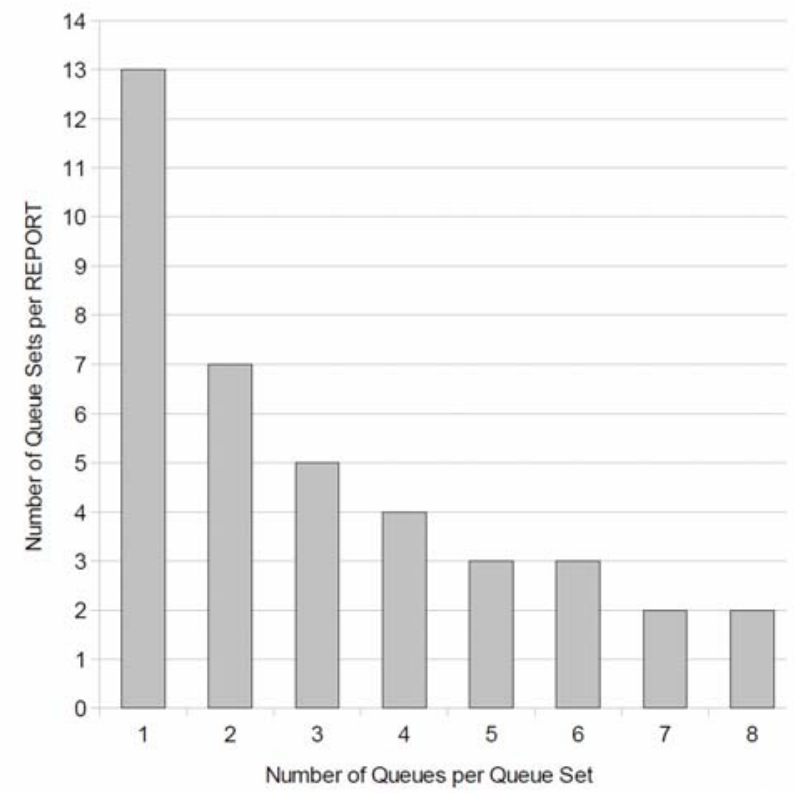

Figure 2-9: Queue sets as a function of Queues

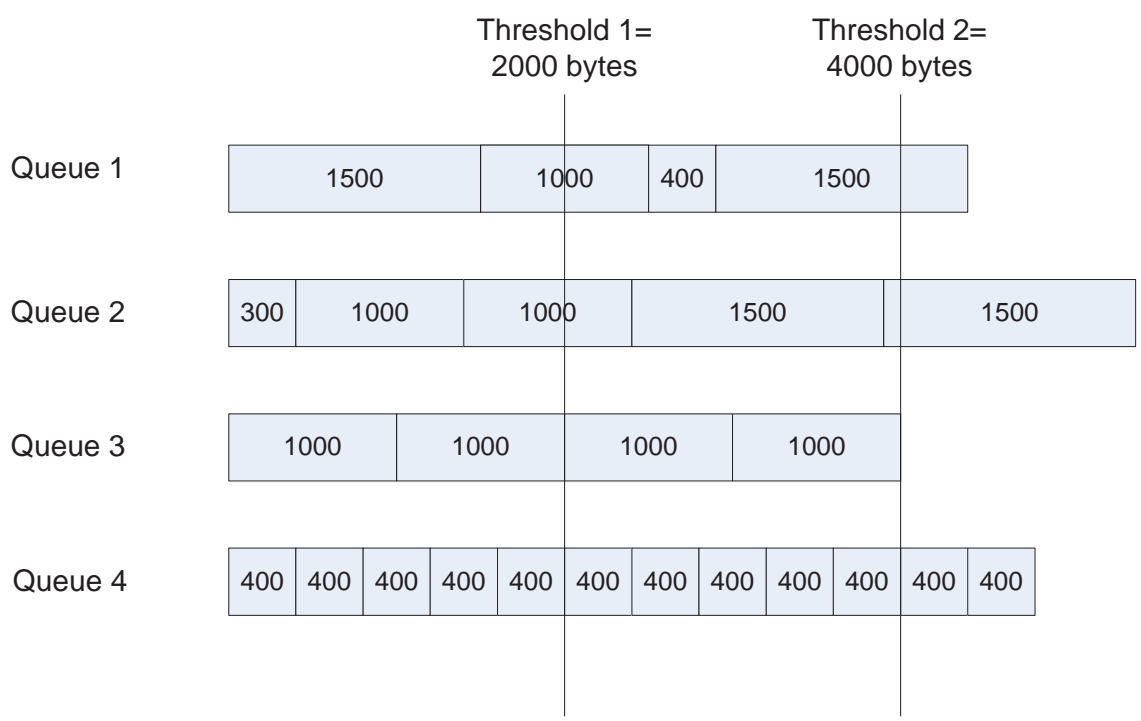

Figure 2-10: Queues with different thresholds 


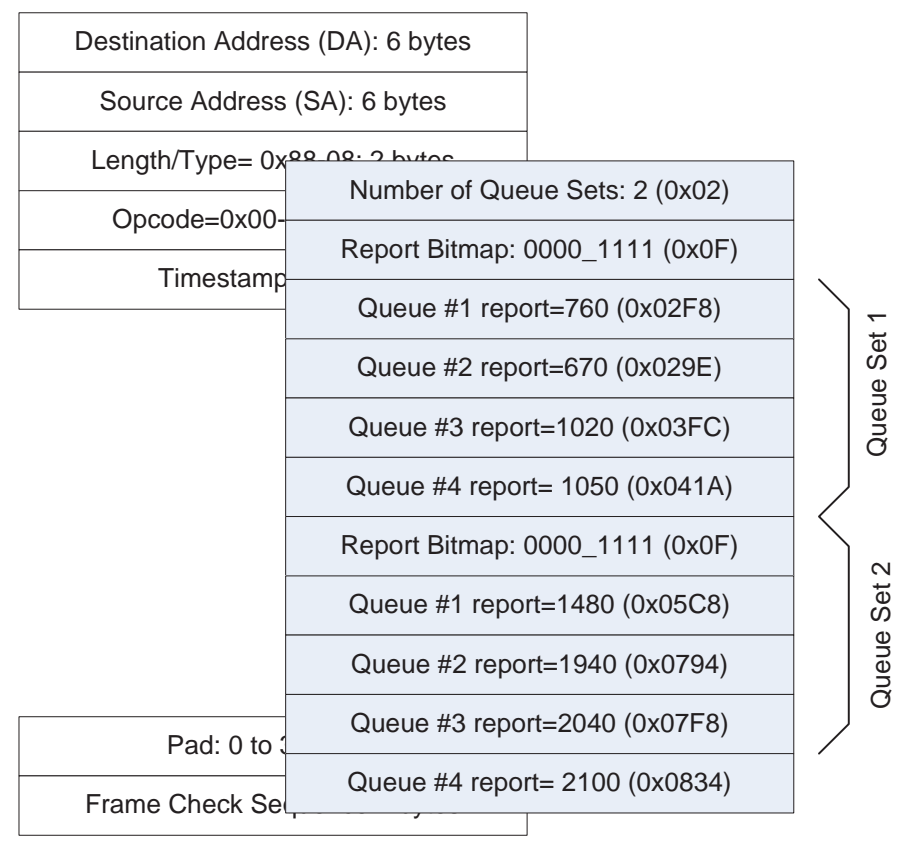

Figure 2-11: REPORT with different queue sets

Every queue report is an unsigned 16 bit number (in units of TQ). Each such report can thus contain the requisition of up to 65536 TQs or $~ 1.05$ ms worth of transmission time. The queue report has to include the time required to transmit the interframe gap and any FEC parity over-head if relevant. Definition of the number of queue sets is not specified in [21]. Its purpose though is to allow the ONU to generate queue reports such that the OLT gets more chance to grant an entire queue length worth of transmission time to an ONU and avoid wasted bandwidth because of packet delineation. For a typical configuration illustrated in Figure 2-10 with two queue sets defined with four queues each the corresponding REPORT is illustrated in Figure 2-11. The numbers in the respective queues indicate the length of the MAC client frame.

The queue reports has to further include the time for the Preamble ( 8 bytes) and Inter-Frame Gap (12 bytes) for each MAC frame transmitted. Thus for the example 
cited, for the first queue set with a threshold of 2000 bytes, the first queue report has $1520(1500+8+12)$ bytes or 760 TQ $(0 \times 02 F 8)$. The second queue set with a threshold of 4000 bytes would result in a report which includes three MAC frames each with its Preamble and Inter-Frame Gap. This totals 2960 bytes or 1480 TQ $(0 x 5 \mathrm{C} 8)$. The other values are calculated likewise and are stated in the figure. REPORT also serves as a keep-alive message from an ONU to an OLT and an OLT has to receive this message at least once every $50 \mathrm{~ms}$ before it de-registers an ONU. The OLT in turn has to transmit a GATE message to every ONU with a time gap of no more than $50 \mathrm{~ms}$. While a GATE message need not have any grants in it for an ONU, it can force the ONU to issue a report with any or every grant.

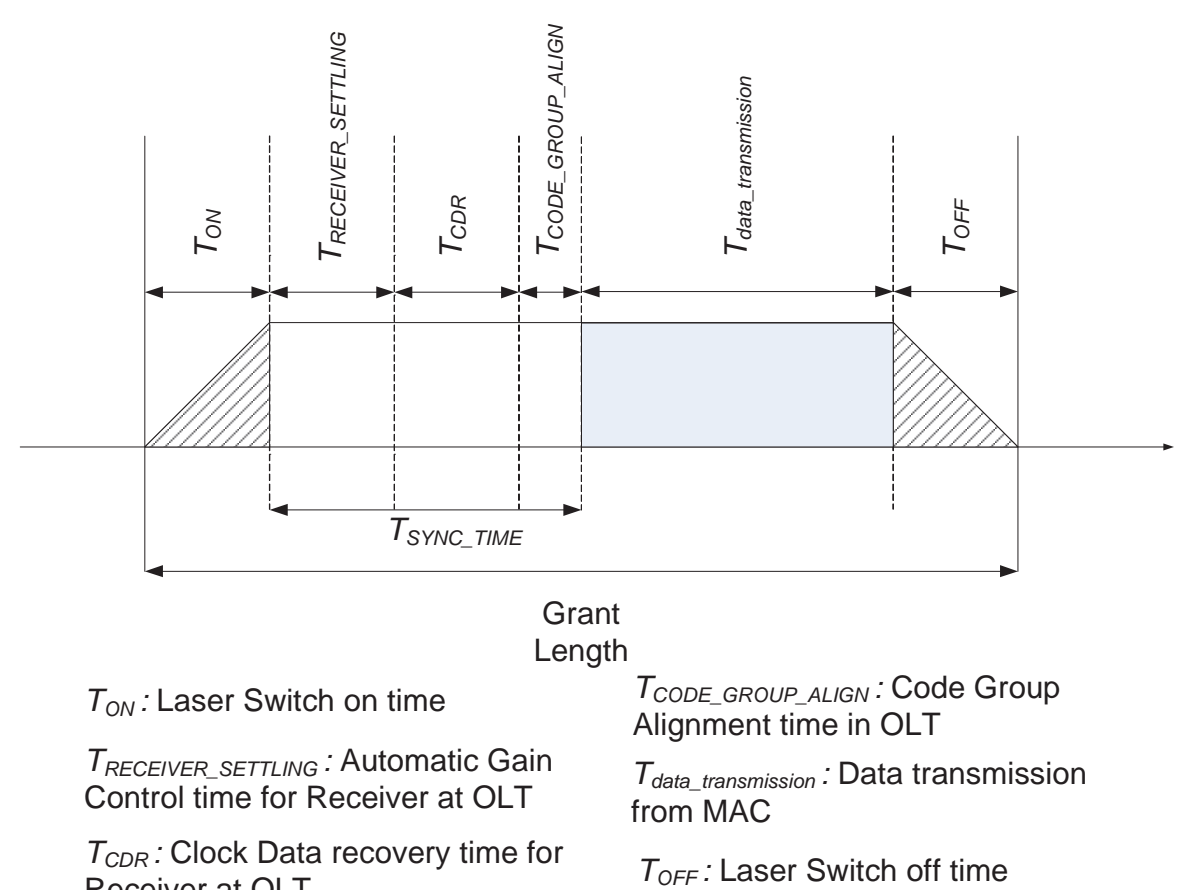
Receiver at OLT

Figure 2-12: Grant Length 
This implicitly means that an OLT has to allocate enough bandwidth to an ONU to transmit at least a REPORT message to indicate alive status. An OLT processes REPORT messages to check the bandwidth demand present in ONUs and allocates grants. The algorithm to give grants is beyond the scope of the standard and is expected to be implemented by the equipment vendor. Different schemes for bandwidth allocation have been subject of wide ranging research [24], [25], [26]. The time line of a typical grant from an OLT is illustrated in Figure 2-12.

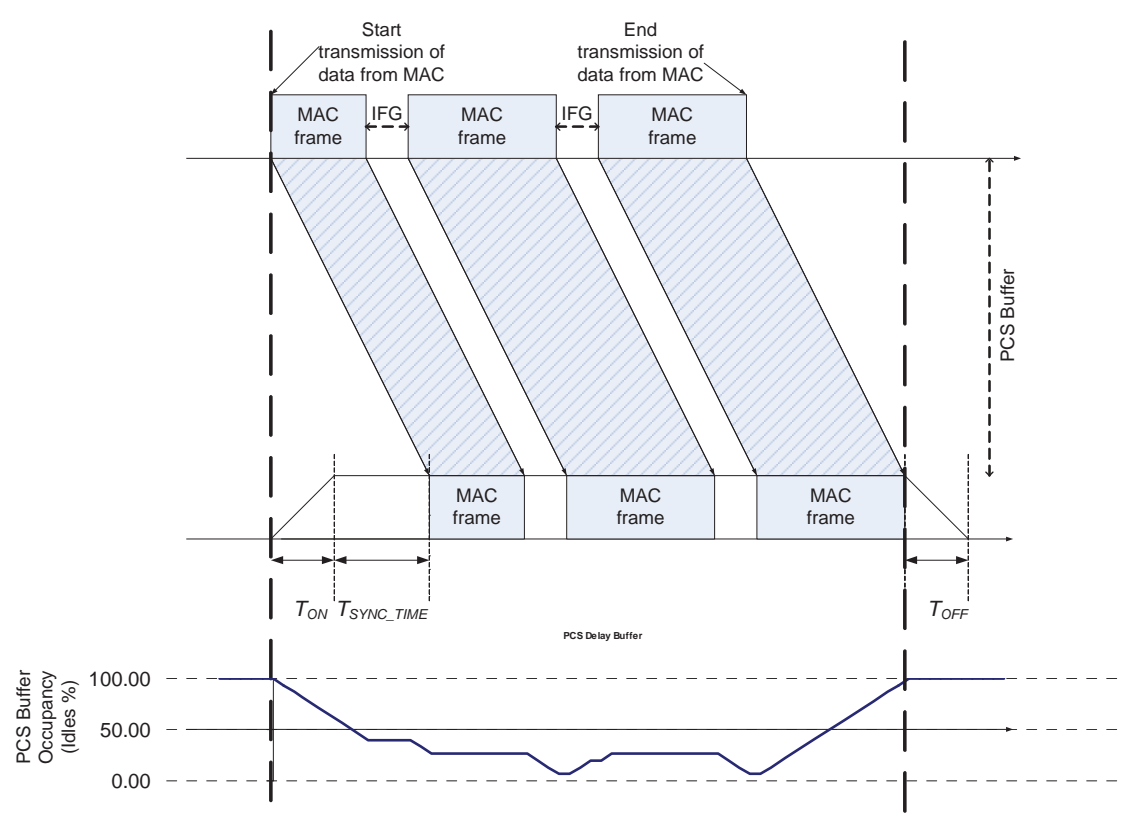

Figure 2-13: PCS Delay Buffer

The time provided in a grant for the ONU laser to turn on and let the receiver at the OLT to synchronize is a necessity in PON operations because upstream transmissions from the ONU are bursty. The PCS is modified to facilitate this kind of operation by introducing a FIFO buffer which acts like a delay line while transmitting MAC frames. At initialization the buffer is filled with idle characters (/I/) and the laser is turned on as soon as the first valid data code groups enter the buffer. If the allocated grant from the OLT is more than what the ONU requires, the ONU starts 
switching off its transmitting laser as soon as there are only idle characters left in its buffer. Figure 2-13 illustrates the buffer occupancy with idles as a function of time and the laser switch on and off times.

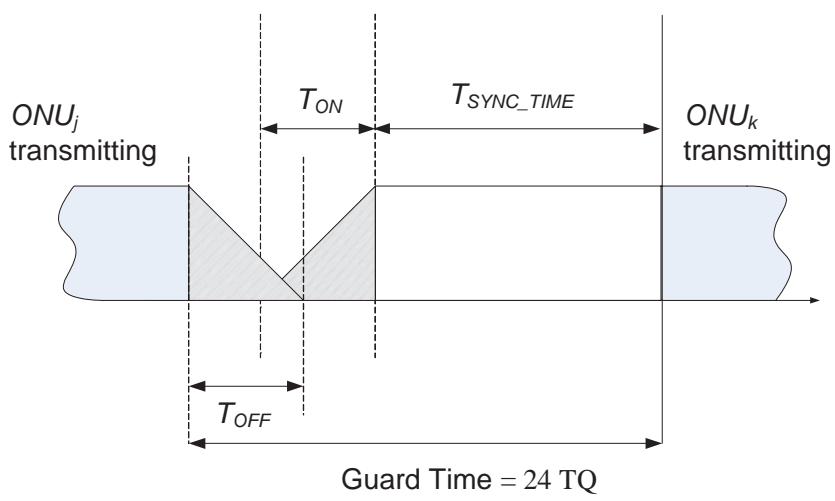

Figure 2-14: Guard Time

The time to turn on the laser $\left(T_{O N}\right)$ is $512 \mathrm{~ns}$. The synchronization time $\left(T_{\text {SYNC_TIME }}\right)$ is a sum total of the time required for the OLT receiver to settle $\left(T_{\text {RECEIVER_SETTLING }}<\right.$ $400 \mathrm{~ns}), \mathrm{CDR}\left(T_{C D R}<400 \mathrm{~ns}\right)$ to lock and for code group alignment ( $\left.T_{\text {CODE_GROUP_ALIGN }}<32 \mathrm{~ns}\right)$. The FIFO buffer should be big enough to allow for this delay (1344 ns = 84 TQ). The synchronization time of the OLT receiver is a negotiated parameter between the OLT and ONU during the discovery process. During this period the ONU transmits idles. The laser switch off time $\left(T_{O F F}\right)$ is again $512 \mathrm{~ns}$, however another ONU can start transmission before the laser is completely switched off as long as the gap between the actual MAC data transmission between them is at least 24 TQ. This is illustrated in Figure 2-14.

While it is expected that the OLT allocates grants which are not overlapping, nothing in the standard prevents it from doing so. An ONU orders grants in order of the start time of the grants. If however the OLT does allocate a grant which overlaps a current grant then the ONU continues transmission till the end of the succeeding 
grant and the laser is not switched off between grants unless there is no data to transmit from the ONU. On the other hand if the end of the succeeding grant is scheduled before the current grant, it is flushed from the list of grants, this is the so called bidden grant.

\subsection{Summary}

The EPON specification has emerged as a leading TDM specification for PON delivery in many markets. This chapter presents a qualitative analysis of the EPON operation in a PON environment. EPON is very similar to Ethernet however has key differences which enable its operation in a PON environment. A PON has a P2MP topology and EPON tries to emulate a P2P connection between an OLT and ONU. The rationale for using an addressing mechanism (LLIDs) which is different from Destination Address (DA) in Ethernet is elaborated. In a PON there is a phase of operation where the OLT tries to find out which ONUs are associated with it, thereafter it tries to range how far the ONUs are. These steps referred to as Discovery and Ranging respectively are important to understand for operation in a reconfigurable BBP like network. The Multi Point Control Protocol (MPCP) operation is analysed to provide understanding of the flow of Operations Administration and Maintenance (OAM) information with EPON as the bearer protocol. It further explains the details of EPON operation including the mechanism of grants by which the OLT allows the ONU to transmit in the upstream direction. The dynamics of the laser turn on and turn off mechanism and its relation to the filling up of the PCS buffer are also analysed.

It is also clearly illustrated that the OLT-ONU association is a dynamic process where it can be re-created after any break in the association. This implies that EPON can be made operational in a dynamically reconfigurable BBP like network where the OLT-ONU association can be time variant. This answers the problem statement of finding out whether or not EPON can be made operational in a BBP like network. 


\section{Chapter 3}

\section{GPON: a comparative perspective with EPON}

PON based access networks have several flavours in terms of the bearer protocol used [27]. Precluding the development of EPON, the first developments in this direction were started by a consortium of network operators with the Full Service Access Network (FSAN) initiative in 1995. The specification developed was ATM based and was called the APON. This was subsequently standardized by the ITU-T under the G.983.1 recommendation in 1998 as the Broadband Optical Access Systems based on Passive Optical Networks (BPON). This and the additional recommendations have been standardized under the G.983.x series [28]. The FSAN initiative to drive this standard to higher speeds and to remove the restriction of using ATM alone as the bearer protocol led to the development of the GPON standards under the G.984.x series first published in 2003 [29]. GPON specification developed on the BPON recommendations and extended the working to higher transmission speeds and used the GPON Encapsulation Method (GEM) in addition to transporting native ATM making it more adaptable to transport of non TDM based traffic. The current standard has removed the specification for ATM as being the bearer protocol for transport. The commercial deployments of EPON and GPON have shown marked preferences in terms of the geographical areas where they have been deployed. Far East Asia has seen preference for EPON while the North 
American market has seen more success with GPON. The Western European market has shown even more preference for GPON [31],[32]. The comparison between EPON and GPON is inevitable and is a keenly debated issue amongst industry and academia. This chapter describes the GPON specifications and provides a qualitative comparison to EPON which was described in the last chapter.

Table 3-1: Downstream and Upstream Speeds in GPON

\begin{tabular}{ll}
\hline Downstream $(\mathrm{Mb} / \mathrm{s})$ & Upstream $(\mathrm{Mb} / \mathrm{s})$ \\
\hline 1244.16 & 155.52 \\
& 622.08 \\
& 1244.16 \\
\hline 2488.32 & 155.52 \\
& 622.08 \\
& 1244.16 \\
& 2488.32 \\
\hline
\end{tabular}

\subsection{Physical Layer}

GPON, unlike EPON, has a legacy of development from frame based TDM systems. The terminology of "Frame" has a different interpretation herein unlike EPON where Ethernet frames are also referred to as "Frames". GPON uses $125 \mu \mathrm{s}$ based uniform frame interval for transmission. The downstream transmission from the OLT is continuous whereas the upstream transmission from ONU is arbitrated and is in bursts. The bursts however are always with reference timing to a "frame" window of $125 \mu$ s which is derived from the downstream clock. GPON supports symmetric and asymmetric combinations of upstream and downstream communication. 
Table 3-1 lists the line rates defined for the upstream and downstream communication specified for GPON. Line rates of transmission defined for GPON are higher than those defined for EPON. Proprietary commercial versions of EPON with $2.5 \mathrm{~Gb} / \mathrm{s}$ line rates are available [33].

The optical specifications for waveband allocations for PON operations and the optical budgets for transmission distance and split rates are derived from [34]. GPON and EPON define diplex transmission over a single fiber. Downstream (DS) transmission is defined in 1480 to $1500 \mathrm{~nm}$ (1490 nm nominal). Upstream (US) transmission is defined in 1260 to $1360 \mathrm{~nm}$ (1310 nm nominal). GPON additionally defines for simplex transmission over two fibers in the $1310 \mathrm{~nm}$ nominal band. The EPON standard does not explicitly specify the type of laser transmitter to be used as long as it meets the specification of spectral width amongst other specifications. GPON defines only SLM lasers for DS. In the US both MLM and SLM are specified with restrictions to $10 \mathrm{~km}$ transmission for $1.24 \mathrm{~Gb} / \mathrm{s}$ and $2.5 \mathrm{~Gb} / \mathrm{s}$ transmission if MLM is used. The $1550 \mathrm{~nm}$ band is reserved for CATV transmission. The use of WDM for operation of multiple channels in the same fiber plant deployment is not an explicit recommendation of either of the standards. Commercial deployments using WDM optics is not very widespread except for some operators [35], [36], [37].

GPON has now defined an enhancement band in the G.984-5 [29] recommendation which is envisaged for Next Generation Access (NGA) networks. Figure 3-1 illustrates the schematic of band usage. In the US additional specifications for Reduced Wavelength Band, with use of SLM lasers only, and Narrow Wavelength Band, with use of WDM lasers only, is defined. Enhancement Band Option 2 is not envisaged for use with Video transmission. There is overlap in some of the bands, CATV transmission (Option 3) is not possible with Option 2, instead Option 4 and Option 5 (not illustrated) should be used if Option 3 is used. The enhancement band is defined only for diplex configuration. 


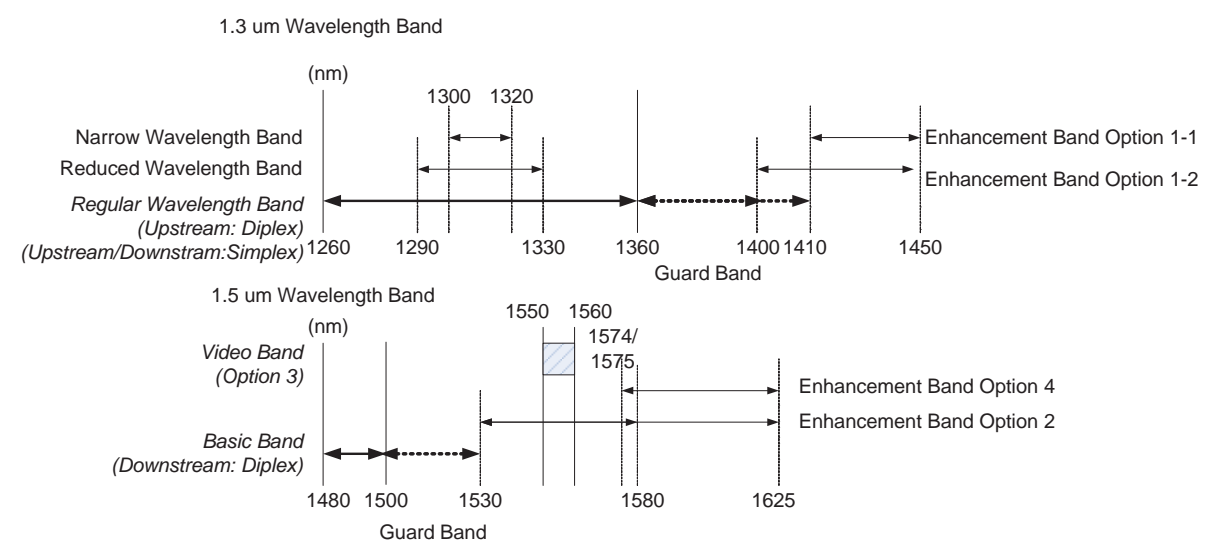

Figure 3-1: GPON Wavelength Bands

Table 3-2: ODN Classes, Split Rations and Reach

\begin{tabular}{lll}
\hline & GPON & EPON \\
\hline ODN Classes & A, B, C & \\
\hline Min Split Ratio & $16 / 32 / 64$ & A, B ${ }^{1}$ \\
Max Physical Reach & $20 \mathrm{~km}$ & 16 \\
& $(10 \mathrm{~km}$ is optional $)$ & $(1000 \mathrm{BASE}-\mathrm{PX} 20)$ \\
& & $10 \mathrm{~km}$ \\
& & $(1000 \mathrm{BASE}-\mathrm{PX} 10)$ \\
Max Logical Reach & $60 \mathrm{~km}$ & Not Defined \\
Max Differential & Logical & Not Defined \\
Reach & & \\
\hline
\end{tabular}

1: Equivalent ODN classes for EPON are mentioned

2: The TC layer is limited to a split of 128

Table 3-2 lists key features related to reach and split ratios for GPON and the equivalent configurations for EPON. The Optical Distribution Network (ODN) classes are derived from [34]. These define the attenuation ranges (Class A: 5-20dB, Class B: 10-25 dB, Class C: $15-30 \mathrm{~dB}$ ) allowed in the fiber plant deployment in- 
cluding optical losses because of splits, splices and connectorization. Split Ratios defined are limited by optical path loss and both standards recommend a minimum number of splits to be supported. Physical reach is the maximum fiber distance between the OLT and the ONU and is limited by physical parameters alone. Logical Reach on the other hand is the maximum distance that can be supported between the OLT and ONU because of restrictions on the distance managed by the Transmission Convergence (TC) Layer in GPON or the equivalent MAC in EPON. It does not include any optical constraints limiting the distance. Differential Logical Reach is the difference in the distance between the farthest ONU and the nearest ONU from the OLT.

The standard does not clearly define the limitations of logical reach, the TC implementation only gives a suggestion of how the logical reach is limited to $60 \mathrm{~km}$. All downstream messages have to be processed within $750 \mu$ s. For a G.652 fiber the RTT for this distance is $600 \mu$ s. ONUs are allowed up to $36 \mu$ s of processing time and this implies that the OLT has a window of at least $636 \mu$ s to process a downstream message in the operational state. As is mentioned later, during the Discovery round ONUs can have an additional random delay of $48 \mu$ s, this window can consequently stretch to $684 \mu \mathrm{s}$. With additional tolerances the figure of $750 \mu \mathrm{s}$ is reached for the processing time. The differential reach however is constrained to limit the disruption during Discovery round and to limit the dynamic range of the OLT receiver.

GPON uses much tighter timings than EPON for laser turn on and turn off times (Laser Turn on and Laser Turn off times in EPON are 512 ns respectively). Table 3-3 lists the ONU laser turn on and turn off times, the Preamble and Delimiter times and the Guard time specified for the different upstream transmission speeds. 
Table 3-3: Physical Layer Overheads (Bits indicated in brackets)

\begin{tabular}{llllll}
\hline $\begin{array}{l}\text { Upstream } \\
\text { Line Rate } \\
(\mathrm{Mb} / \mathrm{s})\end{array}$ & $\begin{array}{l}\text { Laser } \\
\text { On Time } \\
(\mathrm{ns})^{1}\end{array}$ & $\begin{array}{l}\text { Laser } \\
\text { Off time } \\
(\mathrm{ns})^{1}\end{array}$ & $\begin{array}{l}\text { Preamble } \\
\text { Time } \\
(\mathrm{ns})^{2}\end{array}$ & $\begin{array}{l}\text { Delimiter } \\
\text { Time } \\
(\mathrm{ns})^{2}\end{array}$ & $\begin{array}{l}\text { Guard } \\
\text { Time } \\
(\mathrm{ns})^{3}\end{array}$ \\
\hline 155.52 & $12.86(2)$ & $12.86(2)$ & $64.30(10)$ & $102.88(16)$ & $38.58(6)$ \\
622.08 & $12.86(8)$ & $12.86(8)$ & $45.01(28)$ & $32.15(20)$ & $25.72(16)$ \\
1244.16 & $12.86(16)$ & $12.86(16)$ & $35.37(44)$ & $16.08(20)$ & $25.72(32)$ \\
1250 & 512 & 512 & $800^{4}$ & $32^{5}$ & 384 \\
$($ EPON $)$ & & & & & \\
2488.32 & $12.86(32)$ & $12.86(32)$ & $43.40(108)$ & $8.04(20)$ & $25.72(64)$ \\
\hline 1: Maximum & & & & & \\
2: Suggested & & & & & \\
3: Minimum & & & & \\
4: Receiver Settling Time \\
5: Code Group Align Time
\end{tabular}

Unlike EPON where the Preamble has a specific context to a string of bits at the start of a frame transmission, the Preamble herein is equivalent to the sum of the receiver settling time and clock recovery time defined in EPON. The Delimiter is used to enable the OLT to decide start of data transmission and is analogous to the code group align time defined in EPON. Both the Preamble and the Delimiter are suggested values and may be different in implementations. However the sum of the Preamble, Delimiter and Guard times are fixed (205.76 ns, $155.52 \mathrm{Mb} / \mathrm{s} ; 102.88 \mathrm{ns,}$ $622.08 \mathrm{Mb} / \mathrm{s} ; 77.16 \mathrm{~ns}, 1244.16 \mathrm{Mb} / \mathrm{s}$ and $2488.32 \mathrm{Mb} / \mathrm{s})$. In EPON the total Sync Time defined as the equivalent sum of the Preamble and Delimiter is a negotiated parameter and can be much lower than indicated. The guard time is the period between transmissions from two different ONUs.

Differences in physical characteristics of the two technologies are often the key points of discussion in industry circles to show relative superiority of one technology 
over the other. GPON proponents indicate the "inefficiency" of EPON because of the line coding scheme employed. Line coding was necessitated to introduce transitions in data transmission to reduce DC imbalance and help in clock recovery at receiving stations. 1000BASE-X Ethernet standards were specified to use the 8B/10B block codes which were based on similar techniques used for earlier Ethernet specifications. This coding ensures a transition density of at least 3 per 10 bits of code group and a maximum run length of no more than 5 bits. The choice of encoding 8 bits was convenient with byte delineations [38]. The coding scheme does have its limitations for use in higher speed transmissions [39]. EPON carried on with the legacy and also uses $8 \mathrm{~B} / 10 \mathrm{~B}$ coding for transmission. While this allows for re-use of the 1000BASE-X PHY implementations for the block coding, it reduces the so called "revenue" bits to $80 \%$. Some references use the baud rate of transmission in EPON to suggest efficiency of less than 50\% for EPON [40]. This in itself is very simplistic statement as the performance of an access network depends on other parameters like the type of traffic carried and manageability of the network. However there is no debate in the fact that the available line rates can be higher in GPON and thus offer more bandwidth to end users in deployment.

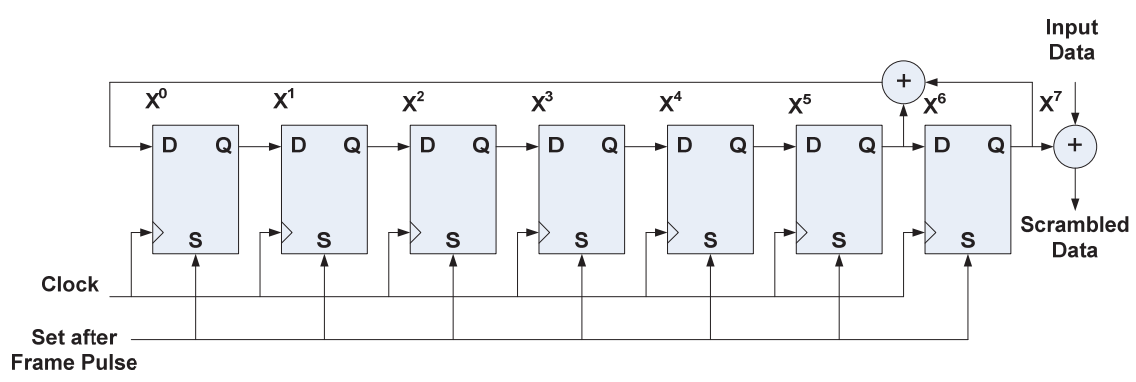

Figure 3-2: Schematic for the scrambler used in GPON 


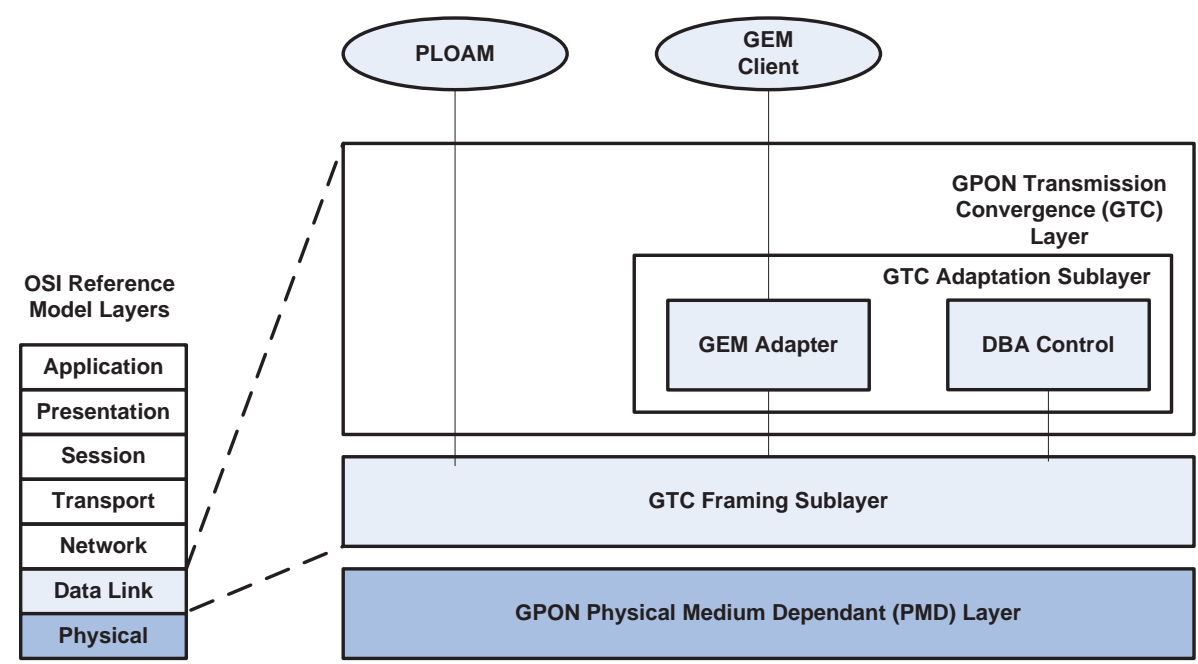

Figure 3-3: GPON Protocol Stack for User data plane

GPON uses Frame Synchronous Scrambling (FSS) which is the same as defined for SDH transmission systems [41]. The scrambler uses a shift register generator with a sequence length of 127 with a generating polynomial of $1+\mathrm{X} 6+\mathrm{X} 7$. The functional diagram of the FSS is illustrated in Figure 3-2. This FSS runs over the entire length of the frame excepting for the frame alignment word. The scrambling operation is discontinuous across frame boundaries because the shift registers are re-set to the initial state with the framing pulse, this in itself does not introduce any limitation [42]. Since scrambling does not add additional bits for transmission as in EPON, the "revenue" bits in GPON are not reduced.

\subsection{Transmission Convergence Layer}

The Transmission Convergence (TC) layer is a layer 2 technology specification for GPON. Figure 3-3 illustrates the protocol stack for the data plane in GPON. Positioning of the OSI stack is given for perspective view. The ITU-T G.984.3 details the recommendation for specification and implementation of the layer. Initial recommendations stated the need for supporting both cell based and frame (TDM) 
based transport by defining the GPON Encapsulation Method (GEM), however the need for transport of ATM has been dropped in the latest recommendation.

\subsubsection{DOWNSTREAM FRAME}

The Downstream Frame is illustrated in Figure 3-4. It is a regular frame based transmission with a periodicity of $125 \mu$ s. The frame consists of a header and payload. The header, called the GPON Transmission Convergence (GTC) Header, consists of the Physical Control Block downstream (PCBd) fields. The payload, called the GTC payload, carries the data meant for downstream ONUs. Unlike other TDM frame based protocols like SDH, the GPON header and the payload area can be of varying length (the overall frame length is fixed at $125 \mu \mathrm{s}$ ).

\begin{tabular}{|c|c|c|c|c|c|}
\hline \multicolumn{2}{|r|}{$125 \mu \mathrm{s}$} & \multicolumn{2}{|r|}{$125 \mu \mathrm{s}$} & & $125 \mu \mathrm{s}$ \\
\hline PCBd $>$ & GTC Paylan & $P C B d$ & CTC Payload & PCBd & \\
\hline
\end{tabular}

Figure 3-4: Downstream transmission of frames with $125 \mu$ s periodicity

The GTC Payload consists of several GEM frames. The GEM frame in turn consists of a GEM Header and the payload area where the raw data frame fragment for the ONUs are carried. GEM frames are uniquely identified by a Port-ID. Unlike EPON where there is a single MAC instantiation in the OLT for every ONU, and a point-to-point connection with a single MAC instantiation in an ONU, the OLT defines several Port-IDs, each of which are mapped to one or multiple ONUs. The OLT-ONU association in GPON is thus flow based. The Port-IDs are used to distinguish logical flows, in EPON on the other hand the separation of the logical flows are not done at the MAC layer but with VLAN configurations. Figure 3-5 illustrates the logical connectivity between the OLT and the ONUs in a GPON network. 


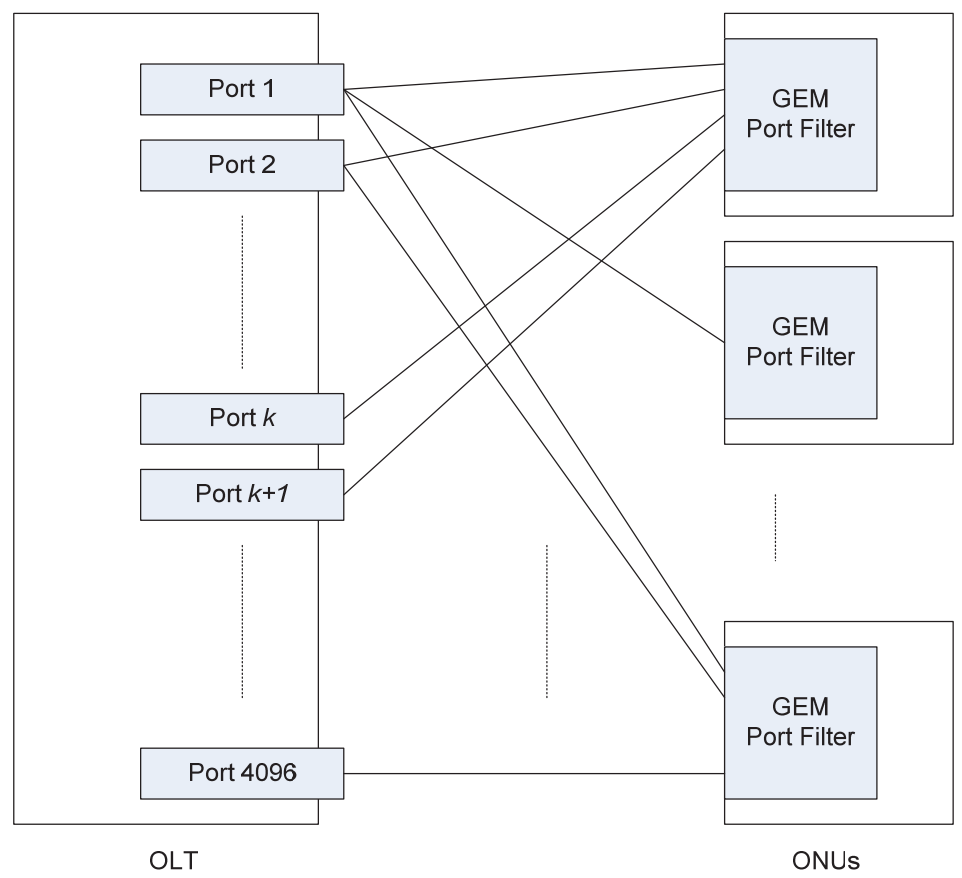

Figure 3-5: OLT -ONU association in downstream

Figure 3-6 illustrates the details of the header in the downstream frame. PSync (Physical Synchronization) is the synchronization field which is unscrambled (0xB6AB31E0). This pattern is used to find the start of frame. Ident field is used to keep a superframe counter for encrypted transmission. Physical Layer Operations Administrations and Maintenance downstream (PLOAMd) as the name suggests is the field used for OAM functionality in the network. 

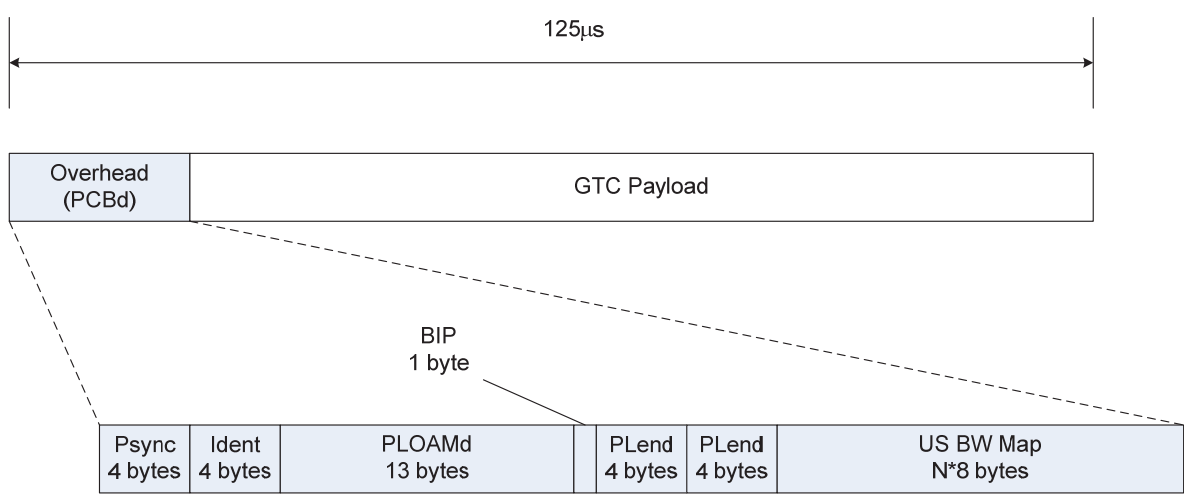

Figure 3-6: Downstream Frame

Downstream Transmission from OLT

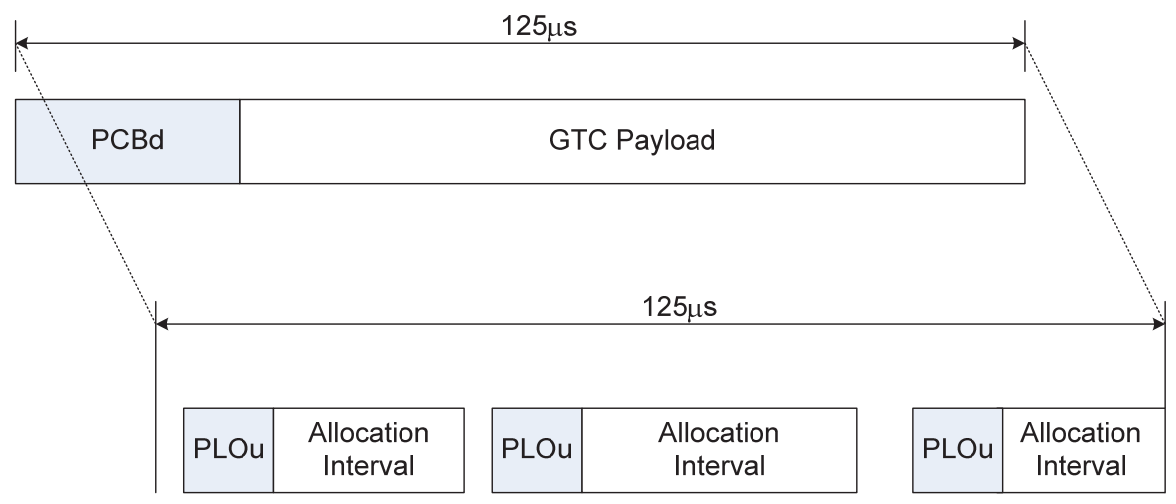

Upstream bursts from ONU

Figure 3-7: Upstream transmission of frames within a frame interval of $125 \mu$ s periodicity

The Bit Interleaved Parity (BIP) field does a BIP-8 coverage over all bits transmitted after the BIP field in the previous frame. The Payload Length downstream (PLend) field indicates the split of the payload area between GEM frames and ATM cells. As in earlier specifications of GPON, ATM was also a defined bearer 
protocol and the payload area could be divided between GEM frames and ATM cells, the dropping of this requirement makes this field redundant but is retained for backward compatibility. The US BW Map (Upstream Bandwidth Map) is the field used to communicate upstream bandwidth allocation to ONUs. Since the US BW Map field is of a variable length, the overhead area is consequently not of a fixed length. However given that the overall frame has a fixed periodicity the payload is then a variable.

Table 3-4: Burst Mode Overhead (Total time indicated in brackets)

\begin{tabular}{ll}
\hline $\begin{array}{l}\text { Upstream Line Rate } \\
(\mathrm{Mb} / \mathrm{s})\end{array}$ & $\begin{array}{l}\text { Burst Mode Overhead } \\
(\text { Bits })\end{array}$ \\
\hline 155.52 & $32(205.76 \mathrm{~ns})$ \\
622.08 & $64(102.88 \mathrm{~ns})$ \\
1244.16 & $96(77.16 \mathrm{~ns})$ \\
2488.32 & $192(77.16 \mathrm{~ns})$ \\
\hline
\end{tabular}

\subsubsection{UPSTREAM FRAME}

Upstream transmission from ONUs are in bursts with respect to a nominal periodic frame interval of $125 \mu \mathrm{s}$. While the periodicity of this interval is identical to that of the downstream transmissions, the frame intervals have an offset with respect to the downstream transmissions. Figure 3-7 illustrates typical upstream bursts from a single or multiple ONUs. Each upstream transmission from any ONU consists of the header and payload area. A single burst from any ONU starts with the (Physical Layer Overhead upstream (PLOu). This consists of the Preamble, Delimiter and the Burst Header. GPON additionally defines a Burst Mode Overhead which overlaps with the PLOu. This includes the Guard Time, Preamble and Delimiter. Figure 3-8 illustrates a typical upstream transmission from an ONU with multiple allocations. The Burst Mode Overhead period is fixed and is listed in Table 3-4. 


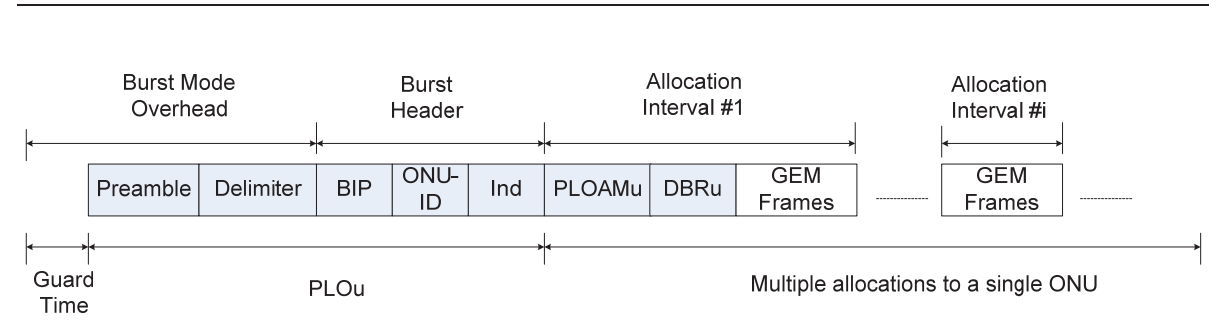

Figure 3-8: Upstream Frame with ONU having multiple allocations

Any optical receiver needs to determine a threshold for decision making before doing clock recovery. A burst mode receiver such as the OLT receiver has to determine the threshold for each burst from every ONU connected to it. The Preamble field is used for this purpose and also to do clock recovery for phase aligning data with the recovered clock. The Delimiter field is used to align the data such that the payload area can be determined without errors. The minimum required field of 16 bits is a derived parameter [29]. Since the total Burst Mode overhead is a fixed value, the Preamble and Delimiter size can vary according to implementations. The recommended values give the optimal burst performance for the receiver [29], [43]. EPON on the other hand has fixed values and fields for the preamble and delimiter.

The burst header consists of a BIP, ONU-ID and the Ind fields. BIP field carries the BIP-8 calculation over the previous frame, ONU-ID carries the ONU identity which is a unique tag allocated to ONUs during registration process. The Ind field contains upstream communication for the OLT. The three most significant bits of this field are used to indicate if the ONU wishes to transmit a PLOAMu, whether the ONU is transmitting with Forward Error Correction (FEC) and presence or absence of Remote Defect Indication (RDI). 


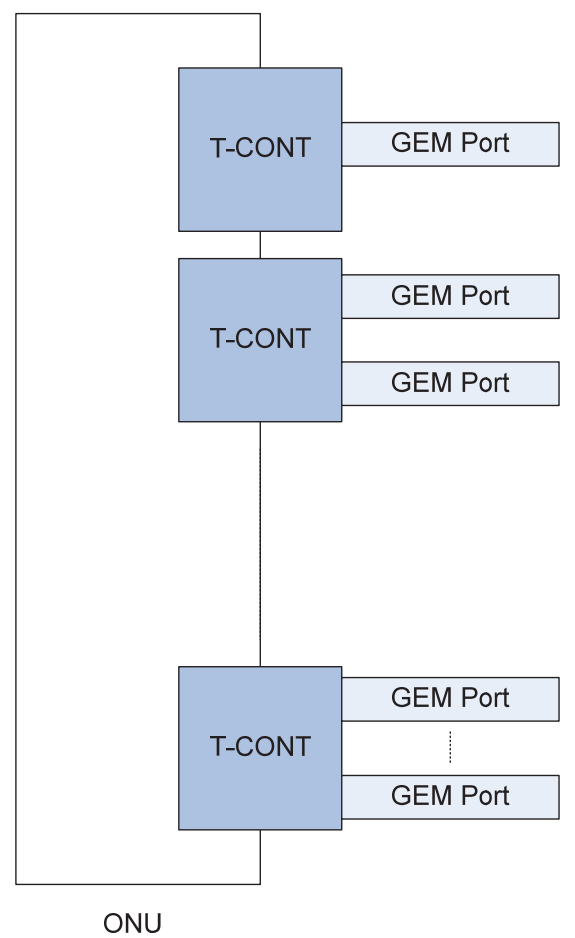

Figure 3-9: ONU Traffic Multiplexing

As in the case of downstream frames the payload is transmitted as GEM frames. The payload area also carries two specific overheads if the OLT instructs the ONU to transmit them during the allocation period. The Physical Layer Operations Administrations and Maintenance upstream (PLOAMu) is similar to the PLOAMd field and is used for OAM messages in the upstream direction. The Dynamic Bandwidth Report upstream (DBRu) is used by the ONU to transmit the bandwidth report. Unlike EPON the allocation time does not include time for transmission of the Burst Mode Overhead or the PLOu. In the upstream direction the allocations granted to ONUs are in terms of traffic units called Transport Containers (T-CONT). Each T-CONT has a unique identifier called the Allocation -ID (Alloc-ID). An ONU can have one or more units of T-CONTs, with each T-CONT 
having one or more GEM ports. The GEM ports are identified by their GEM Port-ID. Thus in the upstream direction there is a two step multiplexing.

Figure 3-9 illustrates the multiplexing structure. Each Alloc-ID is unique in a PON deployment, by default each ONU has at least one Alloc-ID which is the same as the ONU-ID, additional Alloc-IDs can be issued by the OLT per ONU. The GEM Port-IDs are also issued by the OLT to an ONU. Distribution of GEM ports to T-CONTs is the responsibility of the ONU. The GEM ports, which define logical flows, need to be unique for a given ONU, however are not unique across multiple ONUs.

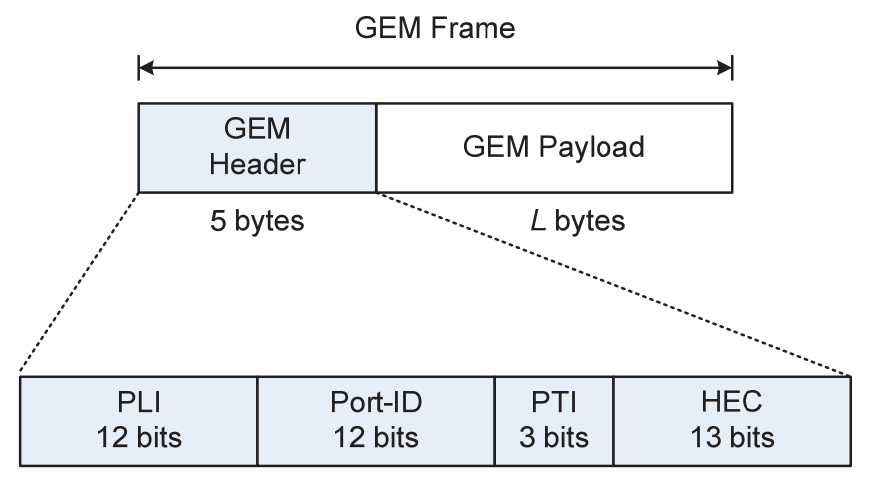

Figure 3-10: GEM Frame

\subsubsection{GEM FRAMES}

Gigiabit capable passive optical network Encapsulation Method or GEM is akin to Ethernet being the bearer protocol for EPON. All user data frames are mapped to GEM frames for transmission in both upstream and downstream directions. The GEM frame has a header and a payload area. The header is fixed to 5 bytes while the payload area can be as large as 4095 bytes. Figure 3-10 illustrates a typical GEM frame. The Payload Length Indication (PLI) field, as the name suggests, indicates the length of the payload. The Port-ID is the traffic identifier for the payload carried. The Payload Type Indicator (PTI) indicates two things, first if the frame is 
carrying user data frames or embedded OAM frames and second, if it is the end of the fragment of either of the frames. The Header Error Control (HEC) provides error detection and control for the other header fields. The GEM header is at the beginning of the payload section and the detection of a correct HEC field indicates a synchronised state to the receiver, thereafter the PLI field is used as an indicator for locating subsequent headers. GEM mapping is more versatile in terms of transport of various kinds of data traffic. Unlike EPON where all services have to be mapped to Ethernet for transport, GEM supports transport of native TDM traffic, Ethernet, TU structures (Tributary Unit traffic entities in SDH transport) and IP (Internet Protocol) frames.

\subsection{Initialisation and Operations}

Functionally GPON is quite similar to EPON in terms of initialisation and operation. As in EPON, the PON goes through a process of Discovery and Ranging before going into operational state where the OLT allocates bandwidth to ONUs for upstream transmission and a downstream broadcast from the OLT which is selectively filtered and received by the ONUs.

\subsubsection{DISCOVERY AND RANGING}

An ONU starts at an Initial state, while it is not allowed to transmit in the upstream direction; the ONU can however transit to a Standby state if it is able to synchronize with PSync received in downstream frames. The OLT has to explicitly activate an ONU for operation. This process takes place through exchange of OAM information through the PLOAM field which is defined for both downstream and upstream transmissions. Figure 3-6 and Figure 3-8 illustrate the position of this field in the respective directions. The PLOAM field is a 13 byte field, details of the field are illustrated in Figure 3-11. 


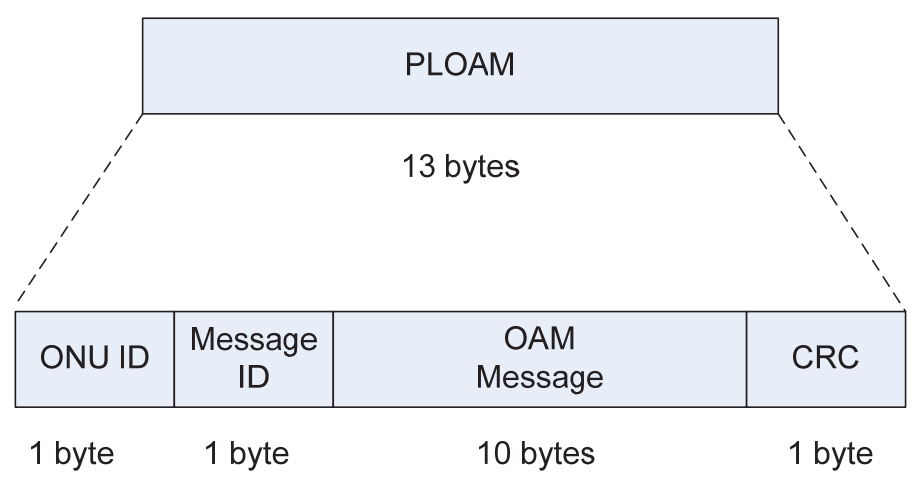

Figure 3-11: PLOAM field

In the downstream direction, eighteen different types of messages are transmitted by the OLT. To initiate ONU activation, the OLT uses a broadcast transmission called the Upstream_Overhead message. The frequency of initiating an activation process is not defined in the standard. The message is used by the ONU to determine the number of preamble bytes to use, the pre-assigned delay $(\mathrm{PrD})$ to use before the ranging process is completed and the optical power level for transmission. In addition to the number of preamble bytes being variable, a key difference with EPON is the ability of the OLT to instruct the ONU to set optical power levels to nominal (Mode 0), $3 \mathrm{~dB}$ lower (Mode 1) or $6 \mathrm{~dB}$ lower (Mode 2). This allows for the OLT to have a lower dynamic range by asking ONUs to adjust the upstream optical power based on the attenuation faced by individual ONUs. On the receipt of the $U_{p-}$ stream_Overhead message the ONU transits to the Serial_Number state. In this state the ONU waits for the OLT to ask for its serial number. If the ONU does not receive any communication from the OLT within a period of $10 \mathrm{~s}$ it transits back to the Standby state. 


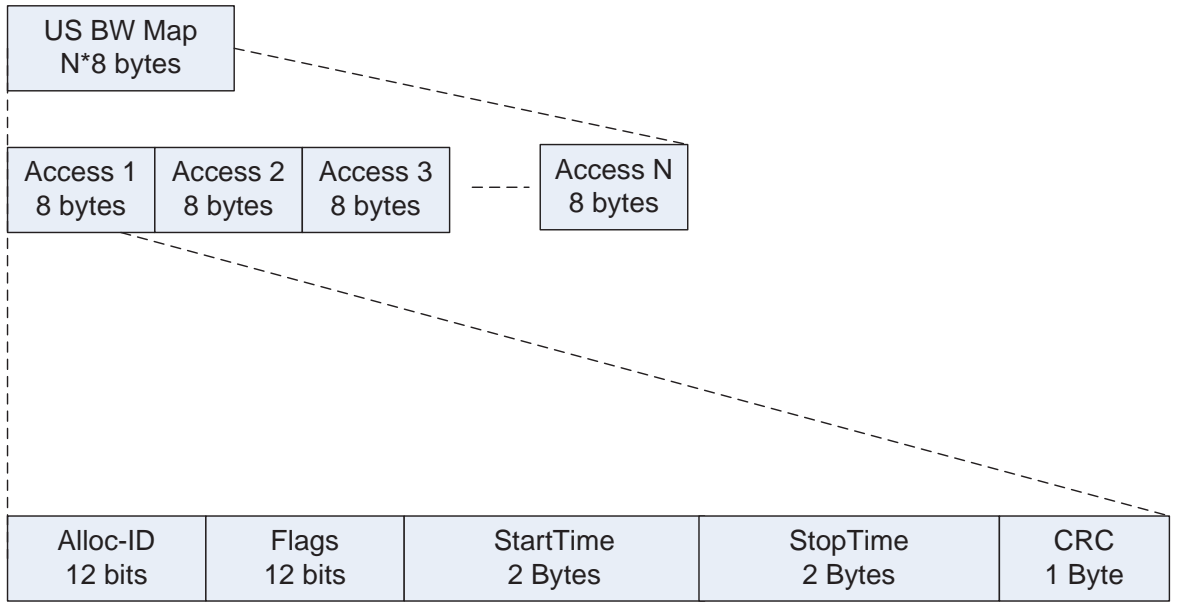

Figure 3-12: Upstream Bandwidth Map

For any upstream transmission, even for activation, the ONU has to receive an upstream bandwidth allocation. The PCBd (illustrated in Figure 3-6) containing the US BW Map field is used by the OLT to communicate bandwidth allocations to ONUs. Figure 3-12 illustrates the US BW Map field. As mentioned earlier, in the operational state each ONU has at least one Alloc-ID defined. The Alloc-ID is a 12 bit number and can take values from 0 to 4096 . The StartTime indication in bytes is the number of bytes after start of frame at which an ONU can start transmission (of a particular T-CONT) and StopTime is the number of bytes after start of frame where it has to stop transmission. Bits in the flag field are set to force the ONU to send a PLOAMu, use FEC and send DBRu messages. The CRC field does a CRC-8 polynomial check over the entire allocation structure and ONUs are expected to drop allocations if there are errors detected. 


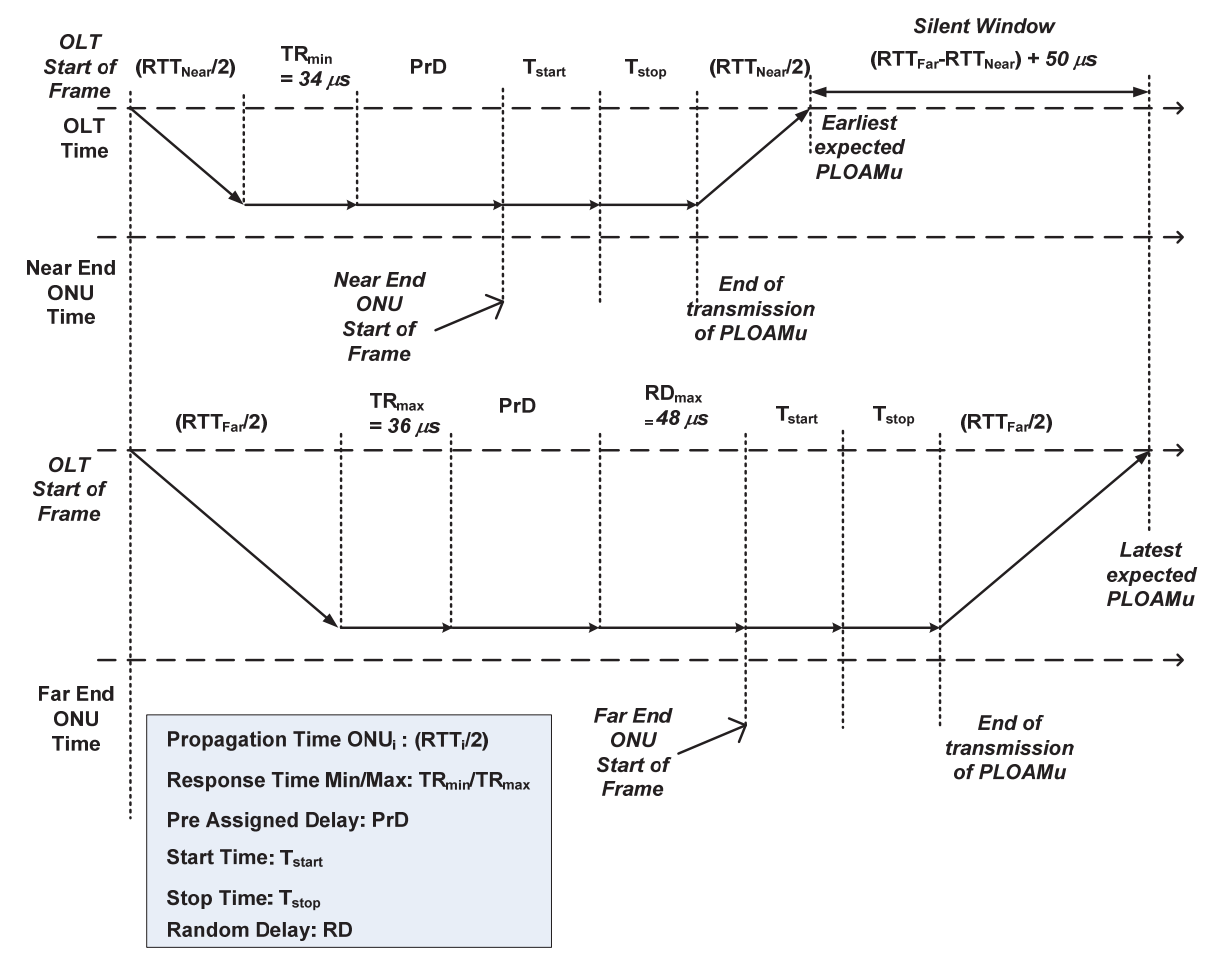

Figure 3-13: Silent window during Serial Number state

GPON uses two distinct processes for discovery and ranging. The first step is exclusively the identification of inactive ONUs alone without any ranging. The OLT allocates bandwidth and creates a Silent Window in which all ONUs in Serial_Number state get a chance to respond without any chance of collisions from regular upstream transmission. The response from the ONU contains the PLOAMu field with its Serial Number identification. The OLT uses a specific Upstream Bandwidth Map (Alloc-ID=254) message by which it grants the necessary bandwidth to the ONUs to transmit the PLOAMu. The OLT also forces the ONUs to transmit the PLOAMu with the necessary flag setting. The grant sets an arbitrary StartTime $\left(\mathrm{T}_{\text {start }}\right)$ and a StopTime $\left(\mathrm{T}_{\text {stop }}=\mathrm{T}_{\text {start }}+12\right)$ which allocates exactly 12 bytes for the PLOAMu transmission. Once an ONU, which is in the Serial_Number state, receives this message, it is allowed a processing delay (TR) of $35 \pm 1 \mu$ s. Subsequent 
to this the ONU has to wait for the PrD which was communicated previously. Since all ONUs which are in the same state can respond at this point, ONUs add a random delay which can be up $48 \mu$ s before which it marks its start of frame reference. This reduces the chances of collision from contending ONUs. The ONU then waits for $T_{\text {start }}$ before transmitting the PLOAMu. Figure 3-13 illustrates this process. At this stage the OLT has no information about the distance to the ONUs in this state and the Silent Window created must account for the differential delay that can be expected between the receipt of PLOAMu from the nearest ONU with minimum response time and no random delay, and from the farthest $\mathrm{ONU}$ with maximum response time and maximum random delay. Thus the window has to be at least 50 $\mu$ s more than the differential RTT. GPON specifies a maximum differential distance between ONUs to be $20 \mathrm{~km}$ and for a G.652 fiber the differential delay in the RTT between Near and Far ONUs can be $200 \mu$ s, thus requiring a silent window of at least $250 \mu$ s.

Subsequent to the receipt of Serial Numbers from ONUs, the OLT assigns ONUIDs (which are also the default Alloc-IDs) through the Assign_ONU-ID message. A successful receipt of this message by an ONU transits it from the Serial_Number state to the Ranging state. ONUs which are unsuccessful in obtaining an ONU-ID use a modulo 3 mechanism $(\ldots \leftrightarrow$ Mode $0 \leftrightarrow$ Mode $1 \leftrightarrow$ Mode $2 \leftrightarrow \ldots)$ to adjust their optical transmission power level and responds at the next opportunity to send its Serial Number. The OLT initiates ranging for individual ONUs that are in the Ranging state by allocation of bandwidth for upstream transmission with the AllocID set to the specific ONU-ID which is being ranged. The process is very similar to the discovery process except that now the response from ONUs are deterministic as only the addressed ONU is expected to respond. The Silent Window required is shorter $(202 \mu \mathrm{s})$ as no random delay is added before transmission. Once an ONU which is in Ranging state receives bandwidth for upstream transmission it transmits its Serial Number in a PLOAMu. This process is used by the OLT to determine the RTT to the specific ONU. 


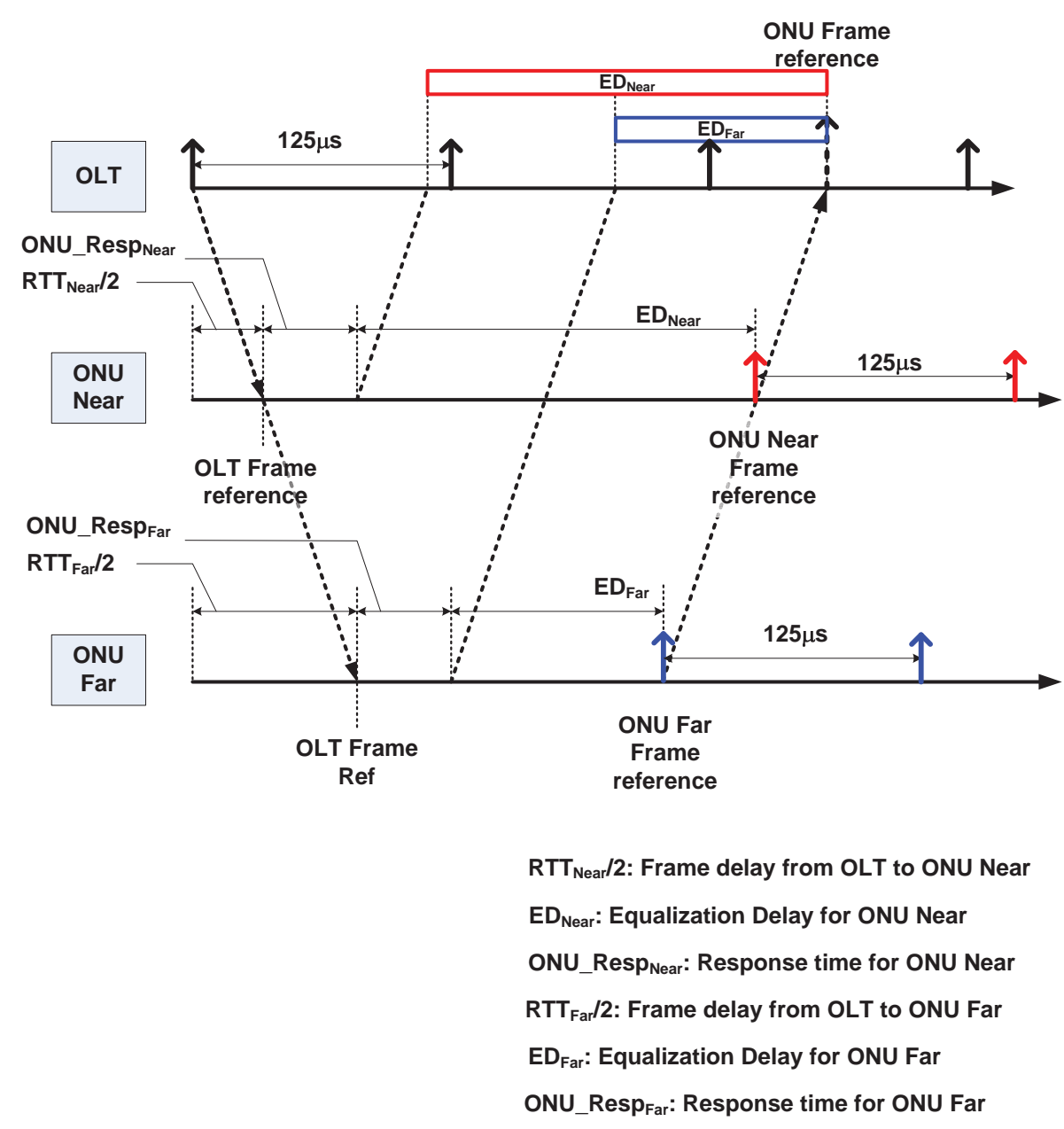

Figure 3-14: ONUs with equalization delay

The OLT completes the ranging process by responding with a Ranging_Time message to the ONU which indicates the equalization delay to the ONU in terms of number of bits. The equalization delay is set such that in the perspective of the OLT all ONUs have the same upstream frame reference. This is very different from EPON where ONUs do not have any time synchronous reference with respect to each other but are synchronized individually to the OLT. Figure 3-14 illustrates the equalization delay assigned by the OLT to ONU Near and ONU Far to keep the 
same frame reference from OLT perspective. The equalization delay in bits is represented by four bytes in the Ranging_Time message and thus theoretically the equalization delay can be as long as 4294967295. At an upstream transmission rate of 2488.32 Mb/s this translates to around $1.73 \mathrm{~s}$ and higher at lower speeds of upstream transmission.

Table 3-5: Max StartTime and StopTime values

\begin{tabular}{lll}
\hline $\begin{array}{l}\text { Upstream } \\
\text { Line Rate } \\
(\mathrm{Mb} / \mathrm{s})\end{array}$ & $\begin{array}{l}\text { StartTime } \\
\text { (Bytes) }\end{array}$ & $\begin{array}{l}\text { StopTime } \\
\text { (Bytes) }\end{array}$ \\
\hline 155.52 & 2428 & 2429 \\
622.08 & 9718 & 9719 \\
1244.16 & 19438 & 19439 \\
\hline
\end{tabular}

Once the equalization delay is assigned an ONU transits from the Ranging to the Operational state. The US BW Map in a single downstream frame contains allocations for upstream transmission within the scope of a single upstream frame period of $125 \mu$ s as viewed by the OLT. The StartTime indication has a minimum value of zero which indicates start of grant at start of frame reference; the maximum value depends on the upstream transmission speed. The StopTime has to be such that the entire allocation is within a frame, hence the maximum value that the StopTime can take is one more than the StartTime. The minimum value for it is likewise zero which indicates no grant. Table 3-5 lists the maximum values for the StartTime and StopTime. The minimum value for both fields is zero. Figure 3-15 illustrates the allocation of bandwidth to an ONU with multiple contiguous and non contiguous allocations. 


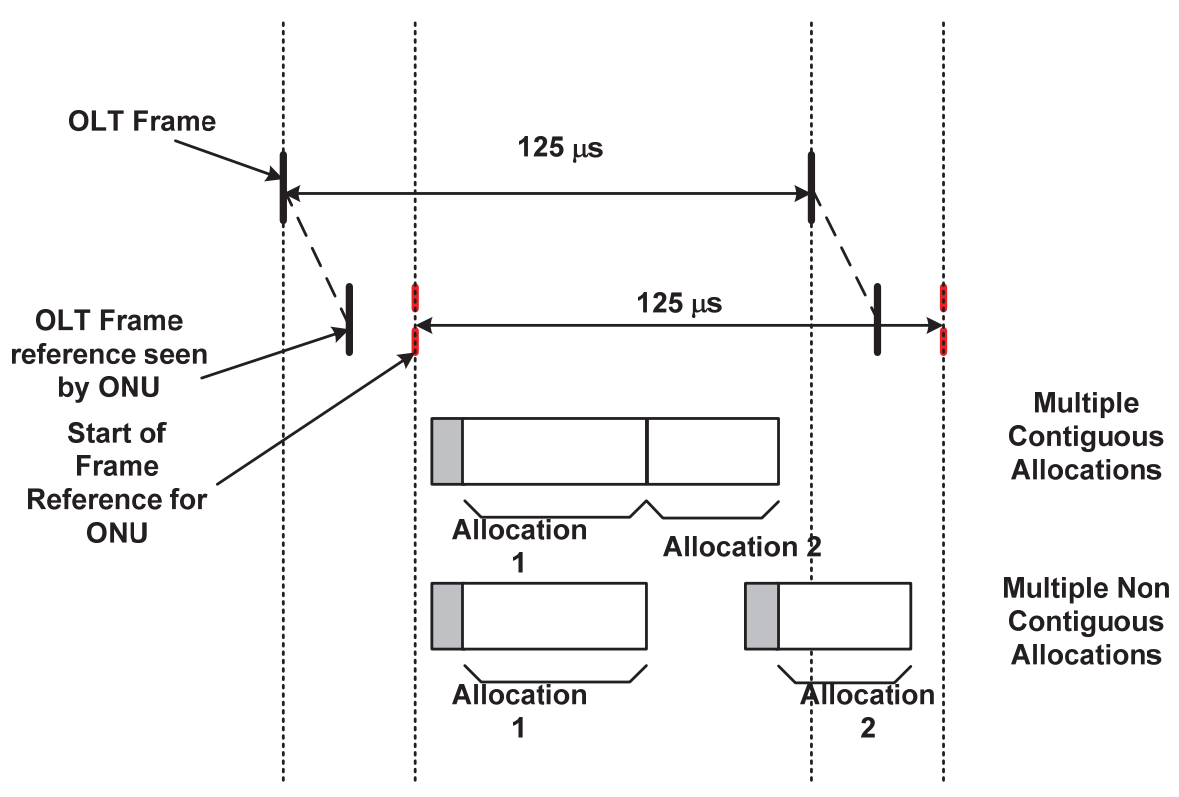

PLOu Overhead

Payload and

other overheads

Figure 3-15: Contiguous and non-Contiguous allocations

\subsection{System Level Aspects and Interoperability with EPON}

GPON supports Alarms and performance monitoring. These diagnostics are important for operational maintenance and service level agreement (SLA) monitoring. EPON lacks this ability and instead has to depend on the Ethernet OAM capabilities which also are not a mandatory feature. In this aspect GPON illustrates its SDH legacy where OAM functionalities are built into the system. EPON, which tries to minimize any changes with respect to the Ethernet frame depends on raw Ethernet OAM capabilities or banks on higher application layers to provide the same. Encryption is another key feature which is not defined in EPON while it is a part of the GPON specifications. The IEEE 802.1 AE MAC Security Standard 
(MacSec) defines security for multi-hop LANs but is not suited for EPON and does not cover the MPCPDU and OAMPDU messages. Proprietary implementations have been suggested and are typically used in EPON.

The aspect of interoperability with EPON was raised as a part of the problem statement. To analyse this aspect we need to first define what interoperability means. In this work, interoperability of EPON and GPON is understood to be the possibility of deploying EPON and GPON within a single network deployment. Both EPON and GPON address the same segment of the network - the access network. Both technologies are similar in the sense that the OLT-ONU association can be a dynamic entity which can be supported if the OLT allows any particular ONU to be logically associated with it. However, it is very clear that the technologies are fundamentally different. EPON is a packet based technology while GPON has its legacy drawn from TDM systems. It is not possible (in practical deployed systems) for a GPON OLT to support an EPON ONU. Likewise an EPON OLT cannot support a GPON ONU. However, as long as a GPON ONU has to be associated with a GPON OLT and an EPON ONU has to be associated with an EPON OLT, it is possible if the specific OLT supports the association. Since the BBP network supports multiple "logical" PONs. It is possible to achieve the simultaneous deployment of EPON and GPON technologies within the scope of a single network deployment as long as they are operating on different wavelength pairs.

\subsection{Summary}

This chapter presents a subjective overview of GPON highlighting the key differ-

ences with respect to EPON. GPON draws upon its TDM legacy and provides key functionalities which are absent in EPON. A comparative study of the protocols should not be restricted to merely calculation of bit rates and frame structures but present the essence of operation and the rationale for use of specific methodology. This is for the very first time that such a study has been presented. The chapter explains how because of the SDH legacy GPON ensures DC balance in bit transmis- 
sion without the need for block coding overheads as are required in EPON. The key phases Discovery and Ranging which are coupled together in EPON are distinct phases in GPON. The chapter further details how GPON allocates bandwidth to ONUs for upstream transmission in the operational state. Unlike EPON which is a packet based technology where an OLT has a specific time reference with every ONU, GPON has a common time reference for all ONUs which is ensured through a parameter called equalization delay. The mechanism for OAM in GPON is presented which uses an in frame communication unlike EPON where distinct OAM packets are used. It explains how an OLT can address multiple ONUs within the scope of a single frame. For instance the PLOAMd can be addressed to a single or multiple (broadcast and multicast) ONUs while the bandwidth allocation is done for a completely different set of ONUs.

Tighter tolerances specified in GPON for laser switch on/off times are mentioned by EPON vendors as a drawback compared to EPON with its more relaxed timing specifications. However GPON specifications, while being more stringent than EPON fall within the realm of commonly available technology rather than being at the edge of it. This in itself does not signify a major drawback for GPON. The other major limitations mentioned by EPON vendors for GPON are the restricted logical range and the strict frame based transport mechanism not being ideally suited for packet transport.

The logical reach limitation is possible to overcome and demonstrations have shown transmission over longer distances [44]. The physical limitations of split and reach dominate much before the logical reach of GPON is reached. Extended range specifications for GPON are available where the physical reach has been increased to 60 $\mathrm{km}$ [45]. The standard allows for increase in differential reach between ONUs to 40 $\mathrm{km}$ [46]. Indeed GPON has evolved to have more flavours than EPON in terms of reach and speeds. The use of TDM based carriers like GPON to carry packetized traffic is a fairly standardized method. However there is fragmentation of packets which makes the OLT and ONUs more complex to implement because the frag- 
mented packets need to be re-assembled before processing necessitating the use of Segmentation and Re-Assembly (SAR) buffers. As mentioned in case of EPON, in GPON too the logical association between an OLT and an ONU can be recreated after a break. This clearly indicates that it is possible to operate GPON within the scope of a BBP like network where the OLT-ONU association can be time variant.

GPON also has seen an evolution towards transmission of IP/Ethernet based transport and the latest standard has dropped the requirement of ATM as being a bearer protocol. The general development of metro and core networks in the direction of packet based transport could benefit EPON as such because Ethernet which already dominates the enterprise and access networks is seeing increased deployment in the carrier networks. In terms of raw bandwidth available GPON outscores EPON but what is important is the service delivery and that is tied to the implementation of the scheduling and bandwidth allocation algorithms [47]. Deployments of EPON and GPON have shown strong regional trends. Both technologies are targeted at the same sector and are therefore not complementary technologies. The question of whether or not they are inter-operable is one of the issues which were part of the problem statement. It is clear that the technologies are not inter-operable per se in the sense that an EPON ONU cannot be associated with a GPON OLT and vice versa. However, if inter-operability is a network deployment is viewed as a case where both technologies can be used within the scope of a single network deployment using the same physical medium for transmission, it is possible in a multiwavelength network. Herein, there can be logical separation between OLT-ONU associations from one wavelength pair to another. While one wavelength pair is used for GPON OLT and ONUs, a different one can be used for EPON OLT and ONUs. Eventual dominance of one, or co-existence of both will depend more on commercial factors rather than technical merits. Out of these two PON technologies, for the first time GPON has outsold EPON in terms of equipment in the first quarter of 2010 [48]. 


\section{Chapter 4}

\section{Case for dynamic reconfigurability in Access Net- works}

The BBP network presents a viable platform to implement a DWDM overlay to existing TDM based commercial deployments like EPON and GPON. The BBP network in addition includes an element of reconfiguration. This chapter details the visualization of the network as switching stage that stretches between the network operator and the end user. The concept of dynamic reconfigurability in such a network is explained and the motivation to have such a network is elaborated.

\subsection{Concept of reconfigurability in an access network}

The over deployment of fiber plant resources is a concern to network operators. An adaptive and scalable network can partly offset the need to over-deploy resources and provide a logical growth path for increase in the capacity of the network. Figure 4-1 depicts the access network as a proverbial last mile interface between the Headend $(\mathrm{HE})$ and the Customer Premises Equipment (CPE). The access network can be viewed as a switch that connects HE based Optical Line Termination (OLTs) equipments to the CPE based Optical Network Units (ONUs). A standard PON deployment would then appear as a static switch with fixed OLT-ONU associations as illustrated in Figure 4-2. 


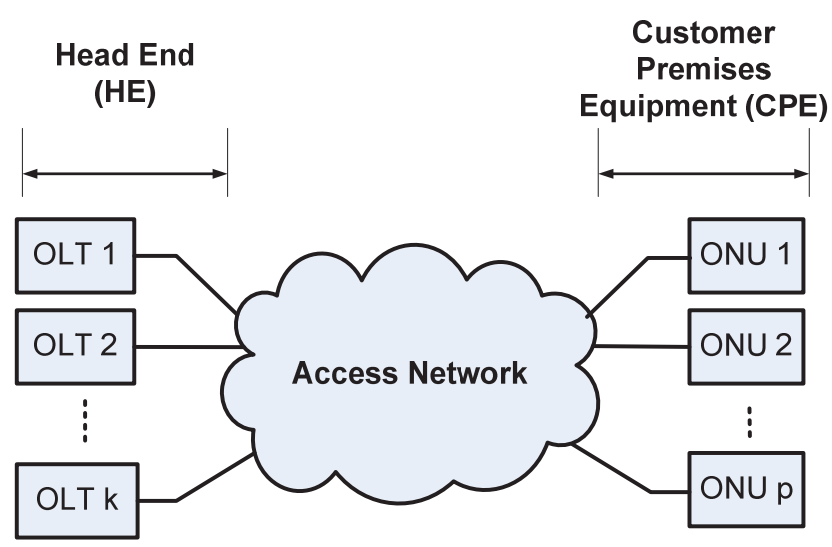

Figure 4-1: Access Network Connecting HE-based OLTs to CPE-based ONUs

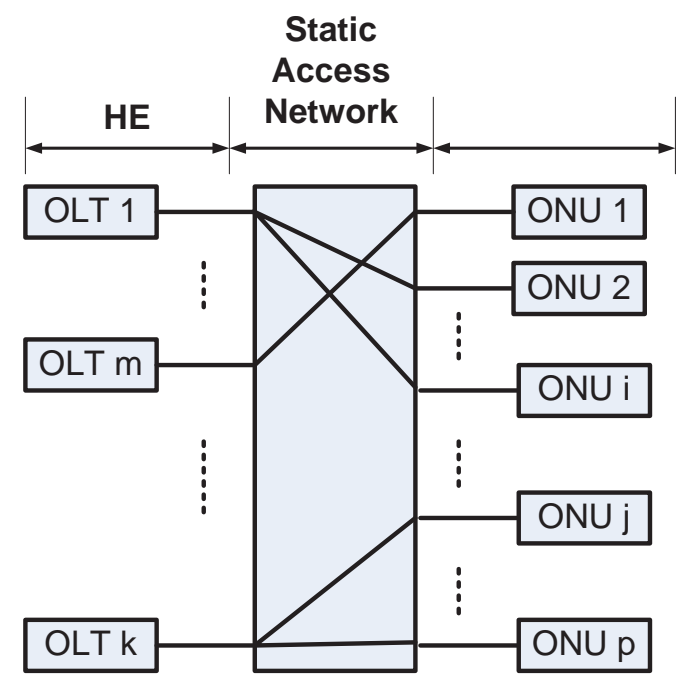

Fixed OLT-ONU association

Figure 4-2: Multiple PON deployments as a static switch between HE based OLTs and CPE based ONUs 
The network is depicted as a static switch where every ONU is always associated with one particular OLT. To increase the aggregate capacity of the network, additional PON deployments have to be made. Each of the depicted PONs therein is independent of the others. Any free capacity in any one of the PONs cannot be used in a practical way in any other PON where there might be need for more bandwidth. To optimise the network deployment, a priori knowledge of bandwidth usage patterns would be needed, and it would be difficult to cater to an evolving scenario where more new users are added or existing users have a changed demand pattern.

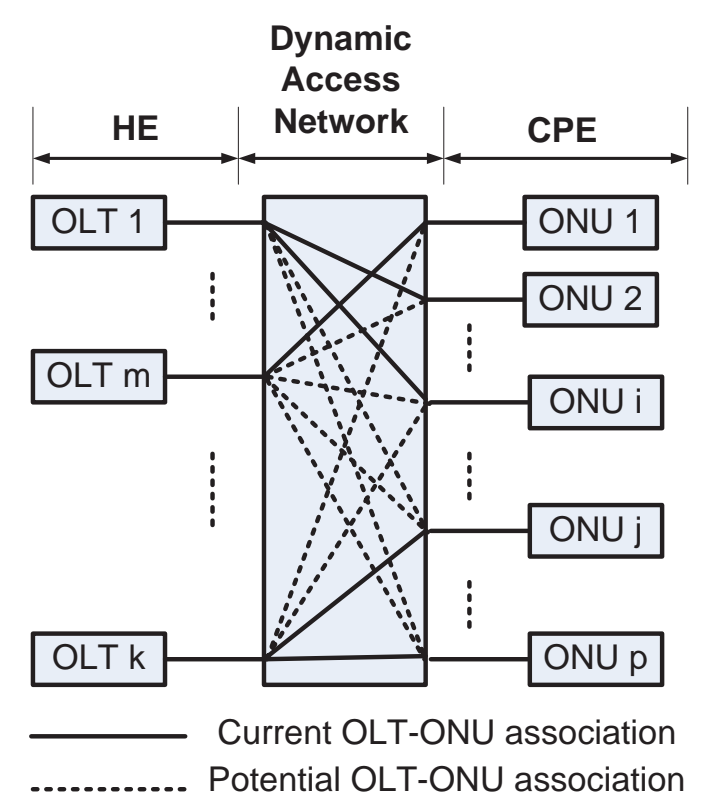

Figure 4-3: Multiple PON deployments as a dynamic switch between HE based

\section{OLTs and CPE based ONUs}

A scalable and reconfigurable network on the other hand, would meet requirements of increasing aggregate capacity in a phased manner and achieve more utilization of the installed network capacity. Use of DWDM to support multiple PONs in a single physical infrastructure deployment is an obvious choice to make the network scalable. In such a network a single wavelength pair supports a logical PON, use of 
multiple wavelength pairs allows for the same fiber plant deployment to support numerous PONs. Unlike a static configuration where an increase in capacity would mean use of additional fiber resources, herein an increase in capacity would mean deployment of additional wavelength pairs without any corresponding increase in use of fiber resources. A single fiber plant deployment would in turn facilitate connectivity between any OLT to every ONU in the network.

Figure 4-3 illustrates the network as a dynamic switch where every OLT can potentially be associated with any of the ONUs in the network. While the need for scalability is addressed by using DWDM to support multiple PONs, reconfigurability per se is not necessarily addressed. One way to address that would be to operate the OLTs on fixed wavelength pairs, and use wavelength agnostic ONUs. The ONUs would then associate with any particular OLT based on the add-drop wavelength pair towards it. The OLT-ONU association would be a dynamic association which can be temporally altered. This would require the use of optical routers, as in the BBP network, that can do selective add-drop of wavelengths towards the ONUs. A detailed analysis for having this reconfigurability is detailed in subsequent sections; a simplistic example nevertheless is presented here to drive the motivation towards such a network. The nominal bandwidth available to an ONU in a logical PON depends on the number of ONUs supported by that logical PON. Increasing the number of ONUs in that particular PON decreases the bandwidth available while decreasing the number of ONUs supported, on the other hand, increases the nominal bandwidth available per ONU. A reconfigurable network would be able to do this and thus allow for load balancing in the network. Figure 4-4 illustrates the concept with just two wavelength pairs. The two logical PONs are depicted as the "Green" PON and the "Blue" PON. In the first configuration the "Green" PON supports three ONUs. The optical router is initially configured such that ONU 5 adds/drops the wavelength pair on which the "Blue" PON operates; this would allow ONU 5 to be a part of the "Blue" PON. If now the wavelength add/drop towards ONU 5 is changed to the one on which the "Green" PON is operating, ONU 5 will stop associating itself with OLT 1 and start associating with OLT 2. This in turn 
would mean that the nominal bandwidth per ONU drops in the "Green" PON while it increases for the ONUs in the "Blue" PON.

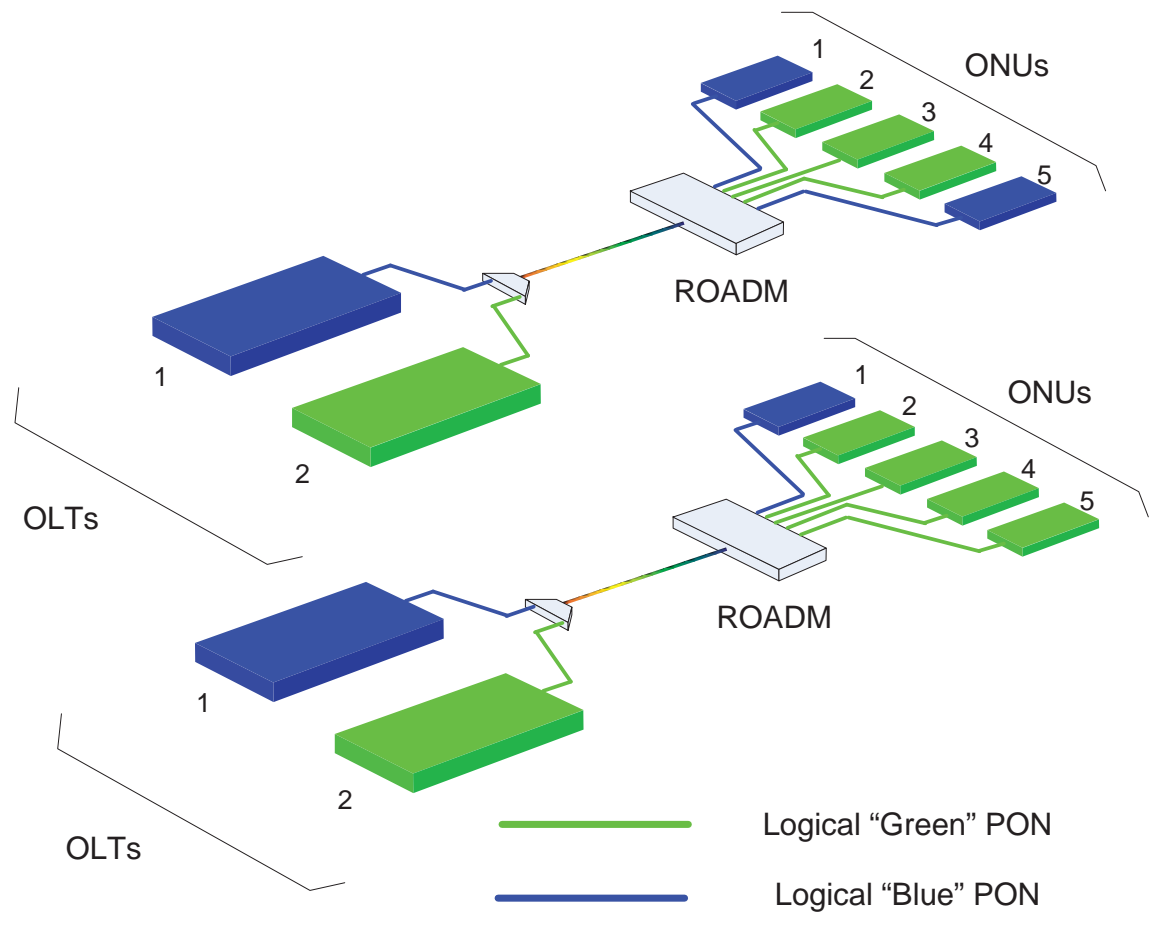

Figure 4-4: Access Network depicted as a two stage switch with two OLTs and five ONUs with ONU 5 changing association from OLT 1 to OLT 2

\subsection{Demand Profiling}

Access networks can show significant variation in diurnal demands [49]. Even during a particular period of the day, there can be variation in the usage patterns by different types of users. The estimation of bandwidth usage in a network thus depends not only on the typical usage by a user but also on the demographics of the types of users served by a network deployment. Studies of typical user profiles are available for the United Kingdom [50]. This study focuses on a scenario-based bandwidth requirement for residential customers in the United Kingdom to forecast progressive growth in residential bandwidth demand and consequently to address the infrastruc- 
ture investment required in this sector. This study has been used as a reference to create a demand profile for people accessing the Internet for work and leisure. The assumption herein is that the demand profile is typically indicative of a typical developed western European society (It should be noted that this kind of profiling depends on the type of society chosen and will indeed differ in a society where internet usage is different). A sample user base to be served by an access network is considered and the typical bandwidth demand that would be generated by users in the network is assessed. Table 4-1 lists the user categories and the distribution of numbers of users in the different categories as adapted from [50]. Eight different user categories (A, B, C1, C2, D1, D2, E, and F) are defined. A total of 128 households is sought to be serviced by an access network deployment. The typical applications used over a day by this customer base are listed in Table 4-2.

Table 4-1: Categories of Users and relative numbers of ONUs

\begin{tabular}{lll}
\hline Category & Description & Number of ONUs (\%) \\
\hline A & Single adult, retired & $19(14.8)$ \\
B & Two adults, retired & $15(11.7)$ \\
C1 & Single male, working & $14(10.9)$ \\
C2 & Single female, working & $8(6.3)$ \\
D1 & Two adults, empty nesters & $17(13.3)$ \\
D2 & Two adults, working & $17(13.3)$ \\
E & Two adults with children & $30(23.4)$ \\
F & Single parent & $8(6.3)$ \\
\hline
\end{tabular}

The applications considered and the bandwidth usages thereof are estimated for the near future. Future applications can be speculative; however, remote data backup, premises, and health monitoring are likely to be in common use. Applications such as peer-to-peer file sharing and remote working are already bandwidth-intensive applications to reckon with today. 
Table 4-2: Applications and Bandwidth Requirements

\begin{tabular}{|c|c|c|c|}
\hline Type & Application & $\begin{array}{l}\text { Downstream } \\
\text { Bandwidth } \\
(\mathrm{Mb} / \mathrm{s})\end{array}$ & $\begin{array}{l}\text { Upstream } \\
\text { Bandwidth } \\
(\mathrm{Mb} / \mathrm{s})\end{array}$ \\
\hline \multirow[t]{5}{*}{ Voice } & PSTN quality call & 0.03 & 0.03 \\
\hline & CD quality call & 0.13 & 0.13 \\
\hline & $\mathrm{DAB} / \mathrm{CD}$ quality audio streaming & 0.19 & \\
\hline & High quality digital audio streaming & 6.00 & \\
\hline & $\begin{array}{l}\text { High quality digital audio fast } \\
\text { download (at twice real time) }\end{array}$ & 12.00 & \\
\hline \multirow[t]{5}{*}{ Video } & CIF quality web conferencing & 0.32 & 0.32 \\
\hline & SDTV quality web conferencing & 0.38 & 0.38 \\
\hline & SDTV video streaming (MPEG 4) & 2.00 & \\
\hline & HDTV video streaming (MPEG 4) & 9.00 & \\
\hline & HDTV download (at twice real time) & 18.00 & \\
\hline \multirow[t]{5}{*}{ Data } & $\begin{array}{l}\text { General web browsing and email } \\
\text { download and upload }\end{array}$ & 2.00 & 2.00 \\
\hline & File down/up load (10 MB in $30 \mathrm{~s}$ ) & 2.67 & 2.67 \\
\hline & File down/up load (50 MB in $30 \mathrm{~s}$ ) & 13.33 & 13.33 \\
\hline & $\begin{array}{l}\text { Peer to Peer down/upload ( } 60 \mathrm{MB} \text { in } \\
30 \mathrm{~min})\end{array}$ & 0.27 & 0.27 \\
\hline & $\begin{array}{l}\text { Remote backup of data ( } 400 \mathrm{~GB} \text { in } 30 \\
\text { days) }\end{array}$ & & 1.25 \\
\hline \multirow[t]{3}{*}{ Others } & Remote monitoring of health & & 0.00027 \\
\hline & $\begin{array}{l}\text { Remote premises monitoring ( } 5 \text { chan- } \\
\text { nel SDTV quality CCTV) }\end{array}$ & & 0.69 \\
\hline & Online Gaming & 2.00 & 2.00 \\
\hline
\end{tabular}

Note: Video category calls include audio content in bandwidth calculations.

Note: The usage of the terms applications refers to a type of service being used rather than as an application in the context of only a software package. 
Table 4-3 : Applications used by different user categories in period of interest

\begin{tabular}{|c|c|c|c|c|c|c|c|c|c|}
\hline \multirow{2}{*}{$\begin{array}{l}\text { User } \\
\text { Cat. }\end{array}$} & \multirow[t]{2}{*}{ Application } & \multicolumn{8}{|c|}{ No. of concurrent applications } \\
\hline & & 1 & 2 & 3 & 4 & 5 & 6 & 7 & 8 \\
\hline \multirow[t]{4}{*}{ A } & HDTV video streaming & 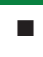 & - & - & घ & & & & \\
\hline & DAB/CD quality audio strm. & & 匹 & 匹 & - & & & & \\
\hline & SDTV quality web conf. & & & 匹 & п & & & & \\
\hline & PSTN quality call & & & & - & & & & \\
\hline \multirow[t]{3}{*}{ B } & HDTV video streaming & 曰 & - & 匹 & - & & & & \\
\hline & General web brows. \& email & & - & $\boldsymbol{\Delta}$ & $\boldsymbol{\Delta}$ & & & & \\
\hline & SDTV quality web conf. & & & & घ & & & & \\
\hline \multirow[t]{4}{*}{$\mathrm{C} 1$} & HDTV video streaming & 更 & - & - & - & & & & \\
\hline & File dn/upload (50 MB in $30 \mathrm{~s}$ ) & & - & - & - & & & & \\
\hline & HQ digital audio streaming & & & घ & घ & & & & \\
\hline & Online Gaming & & & & घ & & & & \\
\hline \multirow[t]{3}{*}{$\mathrm{C} 2$} & HDTV video streaming & - & - & - & & & & & \\
\hline & HQ digital audio streaming & & - & - & & & & & \\
\hline & General web brows. \& email & & & - & & & & & \\
\hline \multirow[t]{2}{*}{ D1 } & HDTV video streaming & - & $\boldsymbol{\Delta}$ & $\boldsymbol{\Delta}$ & $\boldsymbol{\Delta}$ & & & & \\
\hline & General web brows. \& email & & & - & $\boldsymbol{\Delta}$ & & & & \\
\hline \multirow[t]{3}{*}{$\mathrm{D} 2$} & HDTV video streaming & & $\boldsymbol{\Delta}$ & $\boldsymbol{\Delta}$ & $\boldsymbol{\Delta}$ & $\boldsymbol{\Delta}$ & & & \\
\hline & File dn/upload (50 MB in $30 \mathrm{~s}$ ) & & & - & घ & 匹 & & & \\
\hline & General web brows. \& email & & & & & $\boldsymbol{\Delta}$ & & & \\
\hline \multirow[t]{5}{*}{$\mathrm{E}$} & HDTV video streaming & & & $\Delta$ & $\boldsymbol{\Delta}$ & $\Delta$ & $\Delta$ & $\Delta$ & $\Delta$ \\
\hline & SDTV video streaming & & & 匹 & घ & $\boldsymbol{\Delta}$ & $\Delta$ & $\boldsymbol{\Delta}$ & $\Delta$ \\
\hline & File dn/upload (50 MB in $30 \mathrm{~s}$ ) & & & & & - & - & $\boldsymbol{\Delta}$ & $\boldsymbol{\Delta}$ \\
\hline & HQ digital audio streaming & & & & & & & घ & - \\
\hline & DAB/CD quality audio strm. & & & & & & & & - \\
\hline \multirow[t]{2}{*}{$\mathrm{F}$} & HDTV video streaming & - & $\boldsymbol{\Delta}$ & $\boldsymbol{\Delta}$ & & & & & \\
\hline & SDTV quality web conf. & & & - & & & & & \\
\hline
\end{tabular}

Note: Single Instance $\mathbf{~}$; Two Instances $\boldsymbol{\Delta}$ 
The user bandwidth demand during the day will vary depending on the application usage. Typical peaking of demand takes place around 18:00 hours to 21:00 hours [51], [52]. A number of applications from those specified in Table 4-2 are thought to be used by the different user categories in this busy hour as is listed in Table 4-3. Each user in the different user categories is thus considered to use one or more applications during this period. The use of applications by particular user groups is a subjective evaluation that can then be defined as a measurable parameter in terms of bandwidth usage. The number of concurrent applications that are used by any user category is modelled with a heavy-tailed generalized Pareto distribution for each of the categories. The presumption is that most households use fewer concurrent applications, whereas only a few households use many applications in parallel.

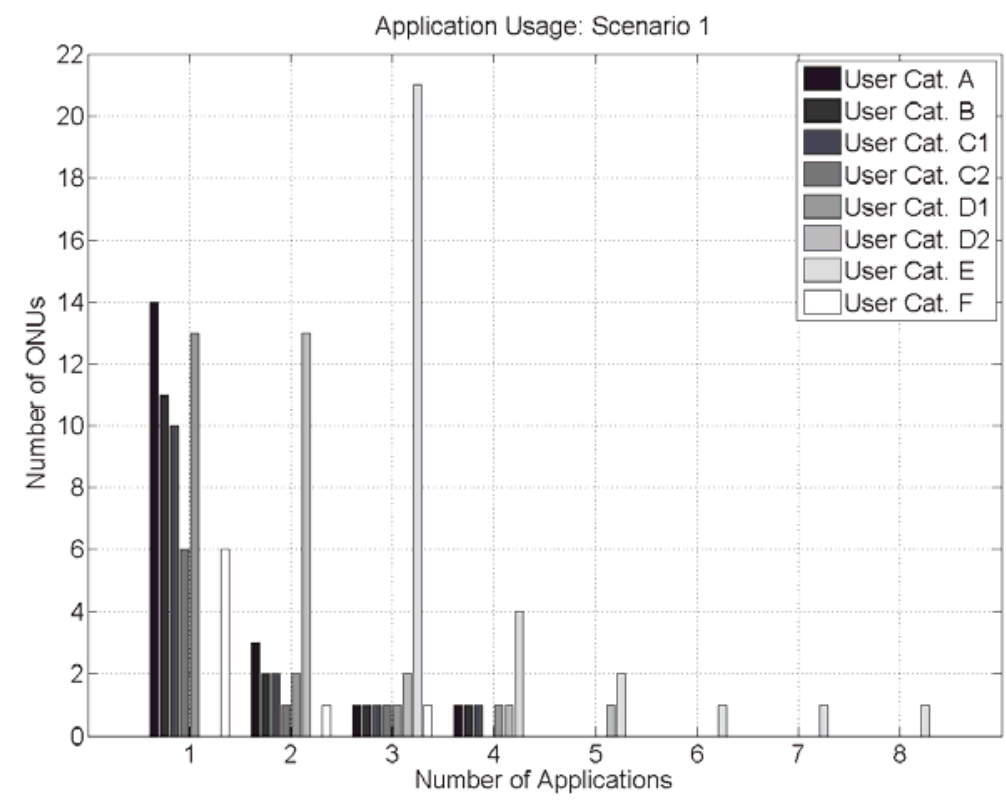

Figure 4-5: Application usage profile by all user categories in Scenario 1.

A bandwidth demand profile called Scenario 1 is generated from an application usage profile illustrated in Figure 4-5 (shape parameter $=2$ and scale parameter $=1$ for the generalized Pareto distribution; Appendix A). The graph represents the number 
of ONUs (each household using only one ONU) as a function of the number of concurrent applications being used. Figure 4-6 illustrates the dynamic range of demands by the different user categories with a weighted mean demand indication by each of the user categories.

\section{Min and Max Downstream Bandwidth Demand \\ $(\mathrm{Mb} / \mathrm{s})$}

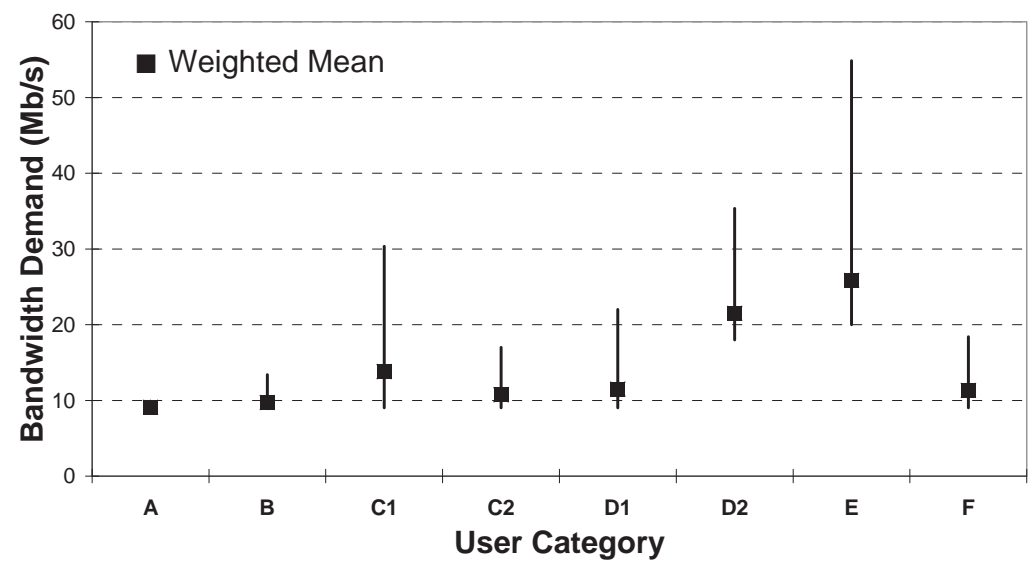

Figure 4-6: Downstream bandwidth demand range by user categories in Scenario 1.

\subsection{Static Network}

In a static network configuration the demand is sought to be met by multiple standard IEEE EPON deployments. EPON specifies a minimum split ratio of 16, however, typical deployments have a split ratio of 32 . This number is considered for a single network group of 32 ONUs being served by a single EPON deployment; for 4 such network groups (128 ONUs) a deployment of 4 EPONs is considered. A network group thus is used in context to define a group of users served by a single such EPON deployment. Two distinct compositions of user types in the network groups are considered. In Network Profile 1, all user categories are uniformly distributed across the four network groups. In Network Profile 2, on the other hand, there is a 
marked skew in the composition with each user group concentrated in one network group. Figure 4-7 and Figure 4-8 illustrate the two network profiles.

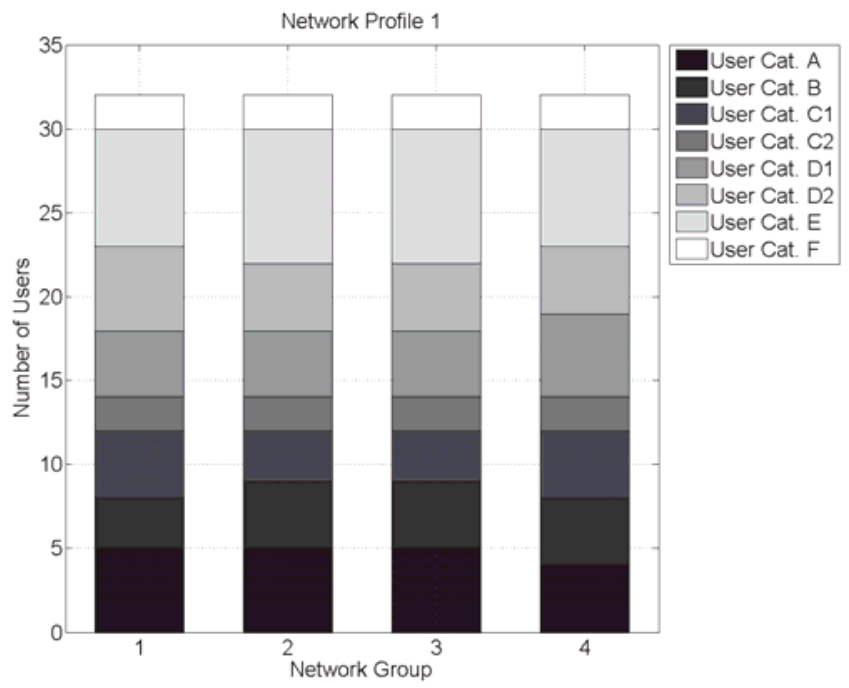

Figure 4-7: Network Profile 1.

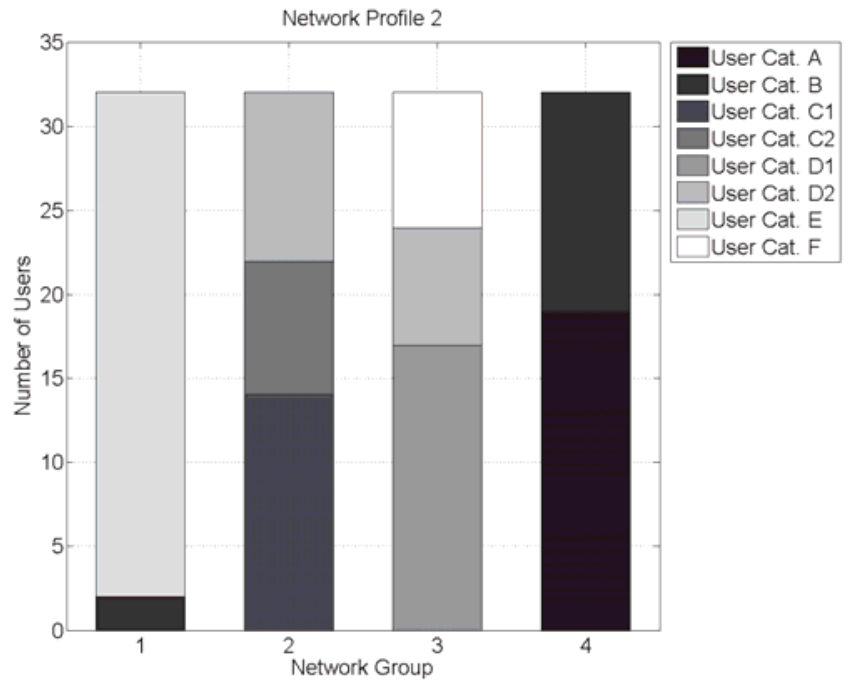

Figure 4-8: Network Profile 2. 
On the basis of the application usage profile in Scenario 1 and the distribution of user types in the two network profiles, the aggregate bandwidth demand can be estimated for each of the four network groups. Figure 4-9 and Figure 4-10 illustrate the aggregate demands in the downstream direction for the two network profiles considered. In Network Profile 1, the aggregate free capacity across all deployments is $38 \%$. The typical network load for each of the EPON deployments is approximately 66\% (the network load is calculated presuming a maximum downstream throughput of $816 \mathrm{Mbits} / \mathrm{s}$ for an EPON with 32 ONUs) [53]. Network Profile 2, on the other hand, shows a marked skew in the demand, with the variation from $37 \%$ to $97 \%$ loading of the network.

The limitations of a static network configuration become evident: even though from an aggregate network perspective there is $38 \%$ free capacity available, it would be impractical to make use of the free capacity from the lightly loaded network groups to meet demands for a loaded network group. Any increase in the demand of users because of change in the usage of applications or because new users have to be served would severely degrade performance for some network groups. To mitigate this limitation, a network operator might need to provision an additional EPON deployment for this group to maintain a desirable quality of service (QoS). Figure 4-11 illustrates the demand in the downstream direction with an additional PON deployment for users in Network Group 1 (now depicted as Network Group 1 and Network Group 5). The aggregate free capacity of the whole network deployment increases to $50 \%$. Yet another limitation of a static deployment is the issue of upgradeability. If only some users would like to have a higher connectivity speeds, an additional network deployment would be required. 


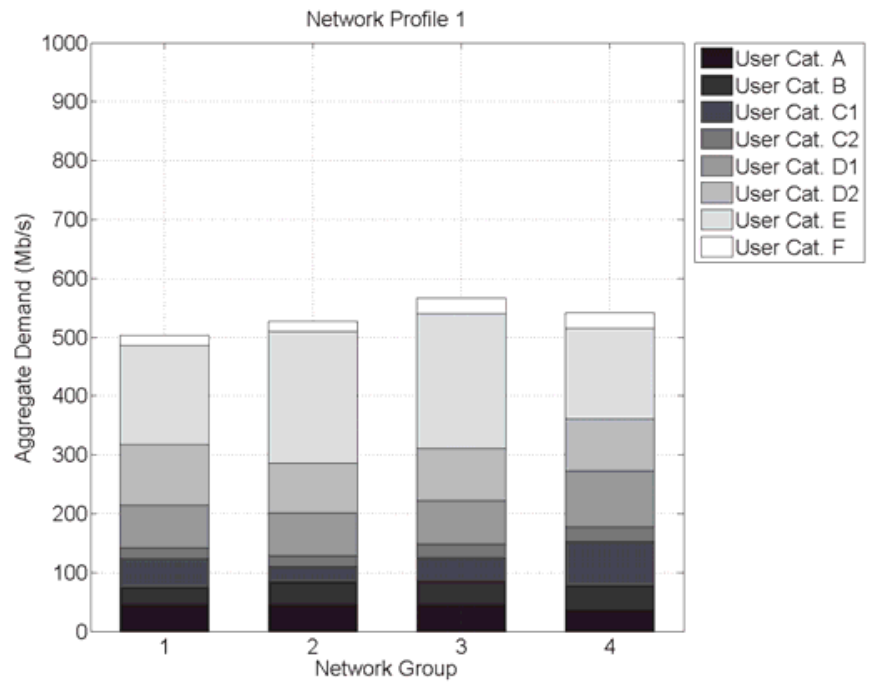

Figure 4-9: Aggregate downstream demand in Network Profile 1.

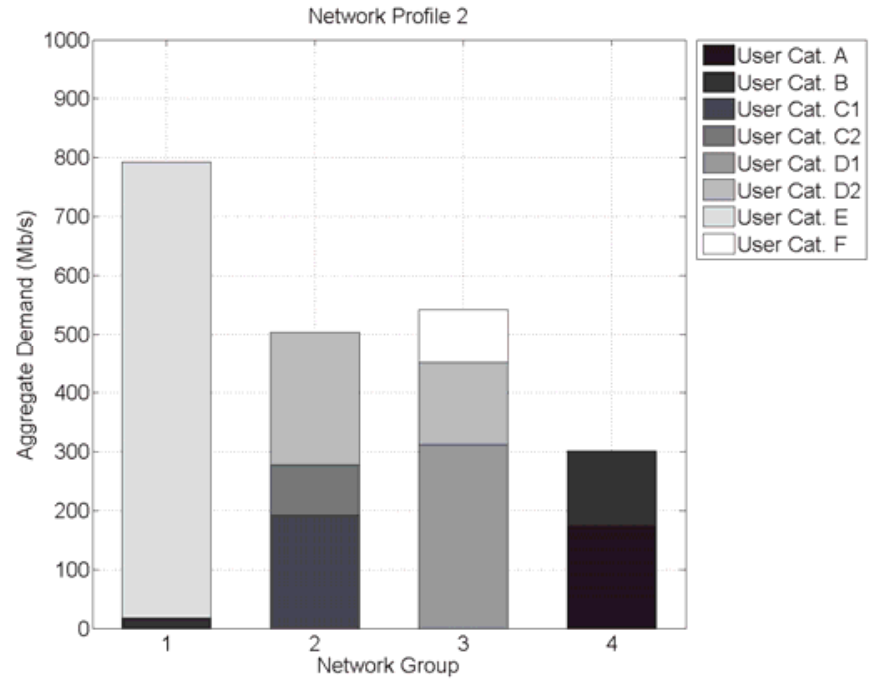

Figure 4-10: Aggregate downstream demand in Network Profile 2. 


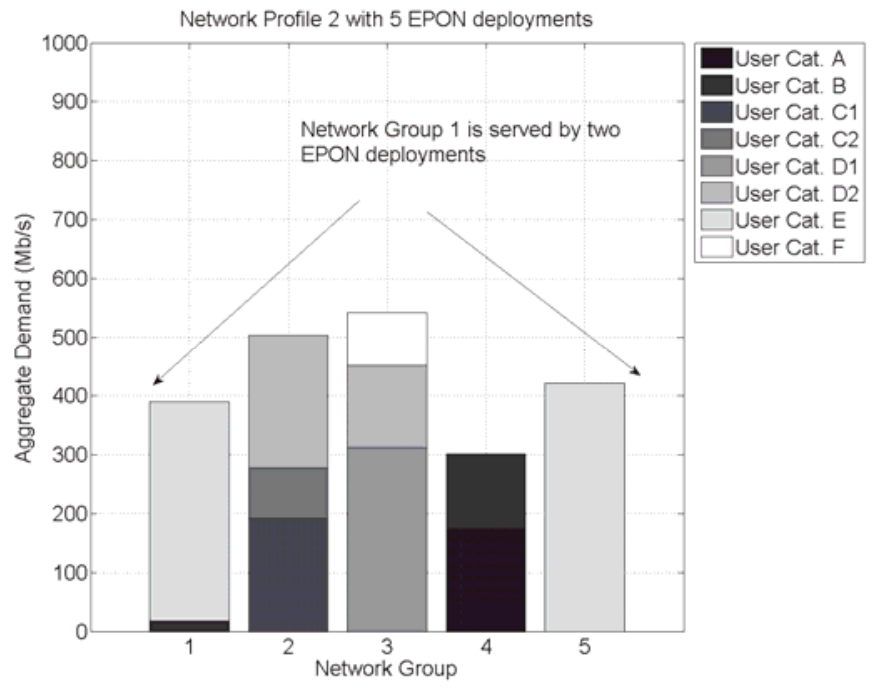

Figure 4-11: Aggregate downstream demand in Network Profile 2 with an additional EPON.

The major limitation of a static network configuration comes from not being able to use up the free capacity in the network with practically implementable means. Of course depending on the types of user profiles and the numbers of users in the different profiles, the bandwidth demands in a network can be different. If the network resources are optimally used up there is little reason to consider a dynamic configuration save for the case when a modular upgradeable path is needed for higher connectivity speeds. If however, resources are not optimally used up, a dynamic configuration on the other hand can be used to do load balancing from a networkwide perspective where instead of multiple fiber plant deployments the network is a single deployment and it is easier to use free capacity of the network where it is needed.

An analogy in real-world terms can be thought of with the problem of parking a big car in a small parking garage. Even if there are several small parking garages available and the cumulative space available across all the garages is enough to park the big car, it would not fit in any one single garage. However, if all the garages could be configured as a single big garage with flexible individual boundaries being redrawn 
to suit the car to be parked, the available space can be more efficiently used both in terms of the number of cars and the size of cars that can be parked in the garage. A dynamic network would be analogous to the big garage with flexible boundaries that can be redrawn to suit the demand presented to it.

\section{$4.4 \quad$ Summary}

This chapter depicts the access network as a switching stage which is an interconnection between OLTs and ONUs. A static access network deployment is one where the ONUs have a fixed association with the OLTs. In a BBP like dynamically reconfigurable network, the ONUs can be dynamically associated with any OLT thus the network appears as a dynamic switch rather than a static one. The concept of managing bandwidth allocation in such a dynamic case is depicted by a simple example with two logical PONs- the "blue PON" and the "green PON". The nominal bandwidth available to an ONU and thus to an end user depends on the number of ONUs associated with a particular OLT. If there are lesser number of ONUs associated to an OLT, the bandwidth available per ONU increases. Likewise, when the number of ONUs associated with an OLT increases, the nominal bandwidth available to an ONU decreases. This concept looks at an inter-PON bandwidth distribution rather than into existing intra-PON static or dynamic bandwidth allocation schemes for distribution of bandwidth within the scope of one logical PON.

The qualification to have a reconfigurable network is then built up by first analysing the bandwidth demand of a typical western European community. User profiles are considered and the typical applications used by the particular profile during a "busy hour" are analysed. The bandwidth requirement for every application is analysed. It is typical that not all users in a particular user profile category make use of all applications concurrently. Most people only use some applications at the same time while a few would be using more number of applications at the same time. A heavy tailed

Pareto distribution is used to consider the number of concurrent applications being 
used by people of a particular user profile. The bandwidth demand for every user profile is thus estimated and the network requirements are dimensioned. It is shown for the particular use case that up to four static PON deployments are required. It is further shown that in case the user profiles are uniformly distributed across the four network deployments, the average bandwidth demand per PON deployment is uniform and if the network is dimensioned correctly there is no issue with bandwidth delivery to the end user. However, if the distribution of user profiles across the different networks is skewed, then some network deployments can be overloaded while others have unused capacity. While the specifics of the distribution of user profiles in different types of communities is beyond the scope of this work what is clear is that in a static deployment, the spare capacity from one network deployment cannot be transferred in a practical manner to the overloaded ones. The issue is of concern when the bandwidth requirements increase over a period of time or there are temporal surges in bandwidth demand. It is shown that additional static network deployments might be needed for as the demand increases, while as a whole, the entire network deployment still has unused capacity. A dynamic network on the other hand would facilitate the usage of unused capacity before there is a requirement to enhance overall network capacity. The scope of study did not address the issue of how the network would behave under different load models. Practical real-world information for this is expensive to obtain as this is requires extensive surveys in target areas and is not commonly available. An interesting aspect would be to extend the current study in this area. 


\section{Chapter 5}

\section{Bandwidth Management Techniques}

In the previous chapter, a conceptual presentation of bandwidth management techniques to optimise the availability of deployed resources in an access network was presented. This chapter details the techniques that can be used to calculate such an optimal network configuration in a dynamically reconfigurable photonic access network.

\subsection{Methodology}

A simplistic view of bandwidth allocation is to view it as a resource which has to be

distributed. Each end user or household in the network has to be allocated part of this resource. Given the fact that the overall network capability in terms of resource availability and facilitation is not infinite, the distribution of resources are subject to certain constraints. In addition to these constraints there is an effort and consequently a "cost" associated with the distribution of this resource. This "cost" is an abstract unit which would typically translate to the operational and the capital expenses for a network operator.

While the unit "cost" of distributing the resource to a particular end user is a known or an estimated value, the bandwidth which is to be distributed per user is as yet an 
unknown value. The overall "cost" of the bandwidth delivery in the network can then be formulated as a sum over all end users of the multiplicative product of the known or estimated unit "cost" of the bandwidth and the yet unknown actual bandwidth allocated per user. This is a linear object function. If the constraints defined can also be formulated as linear functions and are finite, the linear object function can be minimised over a set defined by these linear constraints to determine the unknown bandwidth allocations to an end user. This is the basic tenet of a Linear Programming (LP) formulation [54], [55]. These kinds of LP techniques are commonly used in the so called Routing and Wavelength Assignment (RWA) problems faced in routed WDM networks [56]. Routing refers to finding a light path between a source and destination. Wavelength Assignment deals with finding a wavelength that can be associated with the light path. Typically the problem is split into solving the Routing and Wavelength Assignment separately.

The BBP network offers diversity in the path between the Headend (HE) and every Remote Node (RN). Each OLT can thus have a clockwise downstream/anticlockwise upstream or anti-clockwise downstream/clockwise upstream communication with every ONU. In normal working operation only one of the paths would be used. The routing choice thus is limited to only two paths. The communication direction is decided by minimizing the HE-RN crossings to reach every Customer Premises Equipment (CPE).

The Wavelength Assignment problem in this context can be expressed as deciding on the OLT-ONU association. A heuristic approach can then be taken to solve it [57]. A cost weight is associated for all possible OLT-ONU associations. Every OLT which is commissioned and operational is also allocated a cost weight. The LP technique is thus formulated to reduce the overall cost of allocation of bandwidth which includes the cost of all OLT-ONU associations and the cost of commissioning the required number of OLTs. 
The cost weights for every OLT-ONU association can further be suitably defined to bias the allocation of OLTs to the ONUs if decided by the network operator. Once an OLT-ONU association is formed the cost of this particular association is reduced while increasing the cost of allocation to the particular ONU from other OLTs. This pre-biases the ONU to remain associated with the OLT to which it was originally associated with. The cost weight also decides if certain OLT-ONU associations are possible. For instance an infinite cost weight would not allow certain OLT-ONU associations. This would be done if the OLT-ONU association is physically impossible; for instance if there are optical budget constraints to associate an OLT to a particular ONU, or if the OLT and ONU operate with different protocols like EPON and GPON or with different speeds like the $1 \mathrm{~Gb} / \mathrm{s}$ EPON and the upcoming $10 \mathrm{~Gb} / \mathrm{s}$ EPON. An operator could also decide to constrict certain associations to cater to specific user categories or user locations. On the other hand if a certain OLT-ONU association is always desired the cost weight for that association can be made zero ensuring that the OLT-ONU association always takes place. It should be noted that the technique is distinct from any intra-PON bandwidth allocation scheme that will be operational within the scope of an individual logical PON. The concept of inter-PON bandwidth allocation requires a service aware architecture where a predictive demand from end users can be advertised or estimated [58].

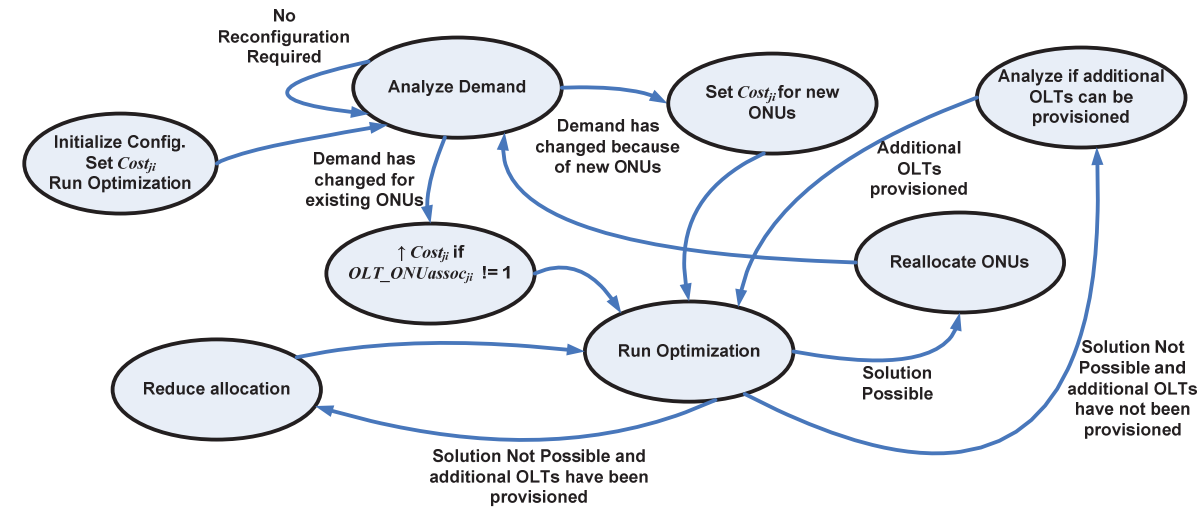

Figure 5-1: State Diagram for Reallocation of ONUs 
It has been shown with pricing models that end users can advertise for a desired bandwidth and specify a tolerable relaxation in the demand. This gives the network operator the flexibility to provision a lesser bandwidth to a user if the situation so demands. The users on the other hand benefit from lower tariffs if they are more flexible in the range specified for the bandwidth relaxation vis-à-vis the desired demand [59]. Figure 5-1 presents the state diagram of the heuristic algorithm used for determining the OLT-ONU association in the network. After initialisation the bandwidth demand of the ONUs is analysed to decide if any reconfiguration is needed. This is also the steady state situation after every reallocation of ONUs. In case the demand changes for existing ONUs such that the bandwidth demands cannot be met in the present configuration, the cost of associating a particular ONU to an OLT, to which it is not currently associated with, is increased and the optimisation routine (subject to constraints) is run to find a suitable configuration for reallocating the ONUs to OLTs such that the bandwidth demands can be met. This is also done in case the demand changes because of the addition of new ONUs. In case no feasible or bounded solution is found for the optimisation routine, the bandwidth allocation to the ONUs is iteratively relaxed to the level specified by them and then an effort is made to find a solution. If a solution is still not found by relaxing the demands up to specified levels, the relaxation is increased in discrete units for all ONUs till a solution can be found. The relaxation comes into play only if the original demand cannot be met or the network operator forces a relaxation in the demand. The algorithm also keeps track of the OLTs commissioned for use and additional OLTs are used if the overall costs thereafter are still lower than relaxing the bandwidth of ONUs.

The parameter and variable declaration for the problem formulation are listed below.

NUM_ONU Number of ONUs supported in the network, $i=1,2 \ldots, N U M_{-} O N U$

NUM_OLT Number of OLTs supported in the network, $j=1,2, \ldots, N U M \_O L T$ 
$B W_{-}$Demand_DS $S_{i} \quad$ Desired Bandwidth demand if the $i$ th $\mathrm{ONU}$ in the downstream direction

$B W_{-}$Demand_US $\quad$ Desired Bandwidth demand if the $i$ th ONU in the upstream direction

$B W_{-}$Relax_DS $\quad$ Relaxation advertised in the downstream bandwidth demand for the $i$ th ONU

$B W_{-}$Relax_US $S_{i} \quad$ Relaxation advertised in the upstream bandwidth demand for the $i$ th ONU

Com_OLT $T_{j} \quad$ Boolean variable to denote whether $j$ th OLT is provisioned

Cost $j_{j i} \quad$ Cost weight to provision an association between the $j$ th

OLT and the $i$ th ONU

Cost_OLT $T_{j} \quad$ Cost to provision the jth OLT

$D S \_a l l o c_{j i} \quad$ Allocation in the downstream from the $j$ th OLT to the ith ONU

Num_ONU $U_{j} \quad$ Number of ONUs that can be associated with the $j$ th OLT

$O N U_{-} D S_{i} \quad$ Total allocation in the downstream for the $i$ th ONU

OLT_DS_capj $\quad$ Maximum bandwidth that can be provisioned in the downstream by the $j$ th OLT

ONU_DS_relax $x_{i} \quad$ Relaxed allocation in the downstream for the $i$ th ONU

OLT_ONUassoc ji $_{j} \quad$ Boolean variable to denote association of $i$ th ONU with jth OLT

$O N U_{-} U S_{i} \quad$ Total allocation in the upstream for the $i$ th ONU

OLT_US_capj $\quad$ Maximum bandwidth that can be provisioned in the upstream by the $j$ th OLT

ONU_US_relax $x_{i} \quad$ Relaxed allocation in the upstream for the $i$ th ONU

Penalty Penalty weight for shortfall in allocation of bandwidth for an $\mathrm{ONU}$ 
US_alloc $j i \quad$ Allocation in the upstream from the $j$ th OLT to the $i$ th $\mathrm{ONU}$

The major constraints are defined as:

a) Each ONU can be supplied by only one OLT

$$
\sum_{j} \text { OLT_ }_{-} \text {ONUassoc }_{j i}=1 \quad \forall i
$$

b) Each OLT can provision bandwidth only if it is provisioned

$$
O L T_{-} \text {ONUassoc }_{j i} \leq \mathrm{COm}_{-} O L T_{j} \quad \forall i, j
$$

c) Total supply to ONUs is equal to the demand that can be met.

$$
\begin{array}{ll}
O N U_{-} D S_{i}=B W_{-} \text {Demand_DS }-O N U_{-} D S_{-} \text {relax } & \forall i \\
O N U_{-} U S_{i}=B W_{-} \text {Demand_US }-O N U_{-} U S_{-} \text {relax } & \forall i
\end{array}
$$

d) Total allocation to ONUs is sum of all allocations

$$
\begin{array}{ll}
O N U_{-} D S_{i}=\sum_{j} D S_{-} \text {alloc }_{j i} & \forall i \\
O N U_{-} U S_{i}=\sum_{j} U S_{-} \text {alloc }_{j i} & \forall i
\end{array}
$$

e) Each OLT can only supply as much bandwidth as it can support in the downstream and the upstream directions

$$
\begin{array}{ll}
\sum_{i} D S_{-} \text {alloc }_{j i} \leq O L T_{-} D S_{-} \text {cap }_{j} & \forall j \\
\sum_{i} U S_{-} \text {alloc }_{j i} \leq O L T_{-} U S_{-} \text {cap }_{j} & \forall j
\end{array}
$$

f) Each OLT can supply only if the ONU is associated with the OLT

$$
\begin{aligned}
& D S_{-} \text {alloc }_{j i} \leq O L T_{-} O N U a s s o c_{i} * O L T_{-} D S_{-} \text {cap }_{j} \quad \forall i, j \\
& U S_{-} \text {alloc }_{j i} \leq O L T_{-} O N U a s s o c_{j i} * O L T_{-} U S_{-} \text {cap }_{j} \quad \forall i, j \\
& \text { g) Each OLT can support a limited number of ONUs } \\
& \sum_{i} \mathrm{OLT}_{-} \mathrm{ONUassoc}_{j i} \leq \mathrm{Num}_{-} \mathrm{ONU}_{j} \quad \forall j
\end{aligned}
$$




$$
\begin{aligned}
& \min \sum_{i, j}\left(D S \_a l l o c_{j i}+U S \_a l l o c_{j i}\right) * C_{0 s t_{j i}}+\sum_{j} \operatorname{Cost}_{-} O L T_{j} * C o m \_O L T_{j}+ \\
& \left.\sum_{i}\left(O N U_{-} D S \_r e l a x+O N U \_U S \_r e l a x\right) * P e n a l t y\right\}
\end{aligned}
$$

The objective function is defined as minimizing the over all supply cost. The supply cost includes the cost to allocate downstream and upstream bandwidth, the cost to commission an OLT and the cost for the penalty for shortfall in bandwidth supply in the downstream and upstream 


\subsection{Demonstration of Methodology}

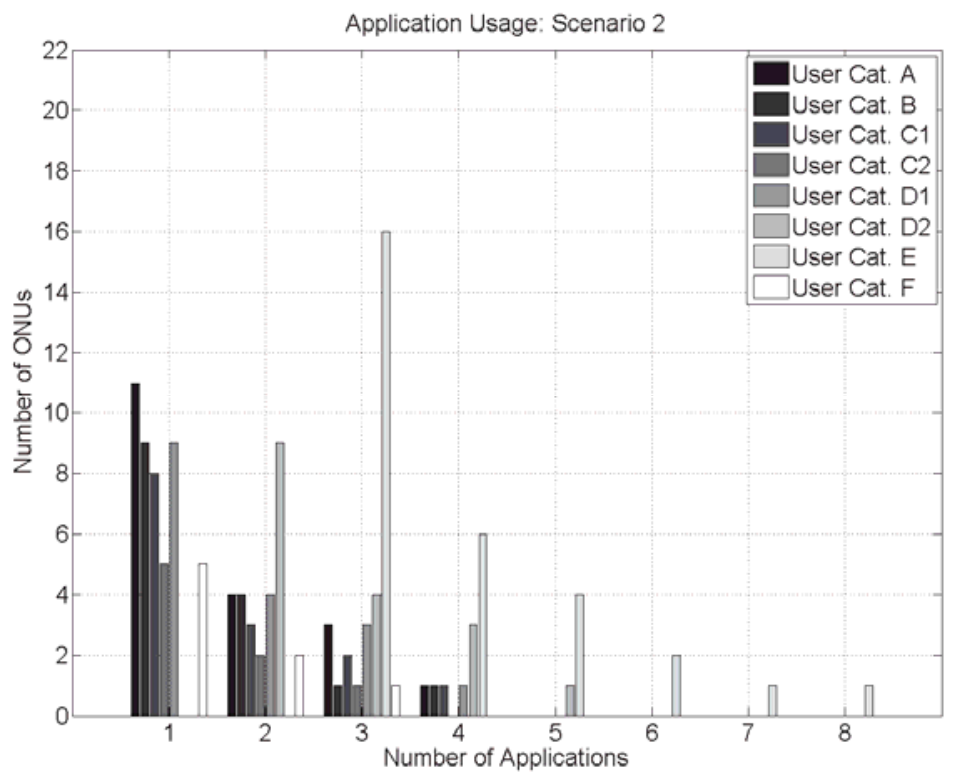

Figure 5-2: Application usage profile by all user categories in Scenario 2

The methodology is studied with respect to an evolving user demand for the same set of 128 end users as seen in the previous chapter. The referral to a network group, as done in the case of a static network configuration, is redundant as potentially all ONUs can be served by any of the OLTs. However, reference to the erstwhile Network Groups 1-4, in Network Profile 2 is retained to simplify depiction in text and figures. An evolving scenario is considered by changing the application usage profile for all the user categories. The new scenarios (Scenario 2-4) are created by changing the scale parameter of the generalized Pareto distribution function from 2 through 4 . The function is used for generating the distribution of the number of ONUs for the number of applications used by each user category. As the application profile changes, the demands of the ONUs are going to change. Figure 5-2 to Figure 5-4 illustrate the Scenarios 2 to 4 wherein increasing numbers of customers are using multiple concurrent applications. 


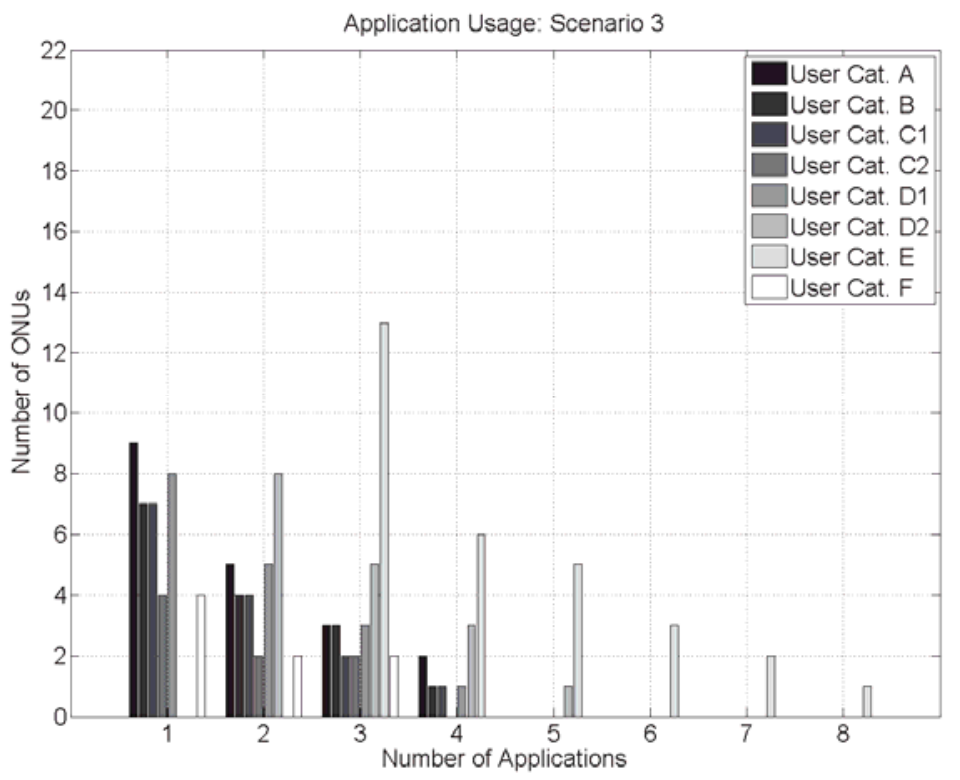

Figure 5-3: Application usage profile by all user categories in Scenario 3

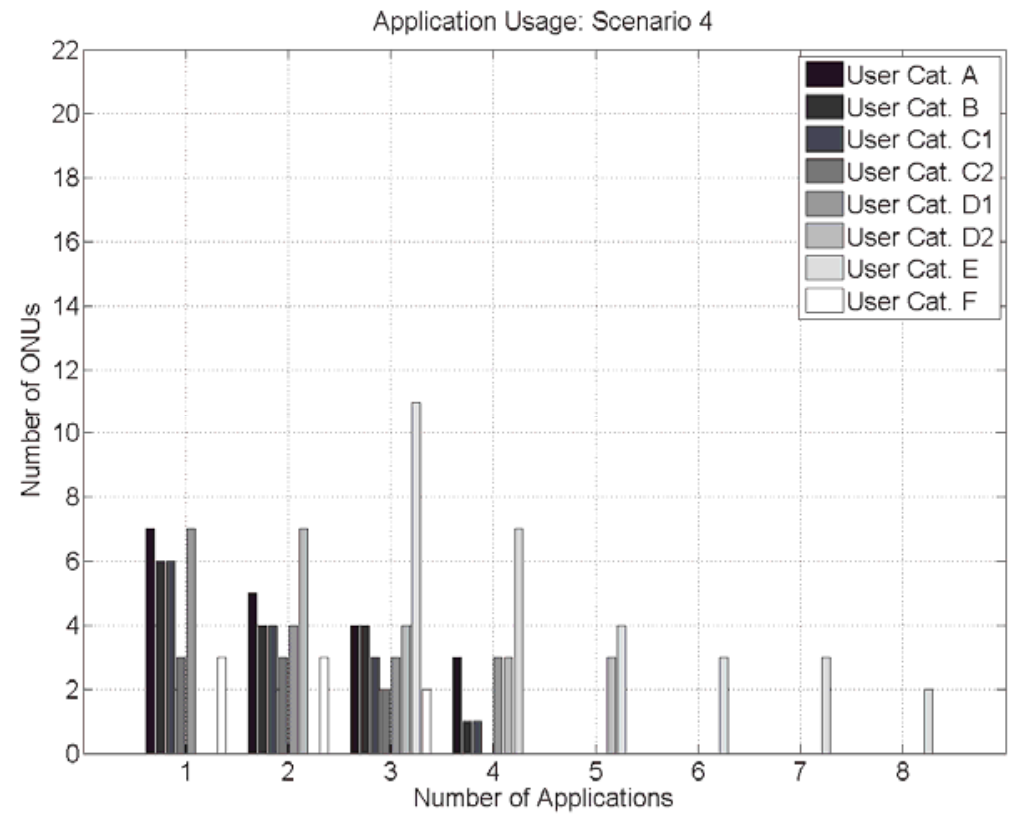

Figure 5-4: Application usage profile by all user categories in Scenario 4 
The demand in the downstream direction per ONU from Scenario 1 to 4 is illustrated in Figure 5-5 to Figure 5-8. Aggregate bandwidth demands depend on the number and type of applications used by different end users. As more users tend to use more concurrent applications, the aggregate demand increases. The initial demand profile as illustrated in Figure 4-10 required the use of at least four standard EPONs and it was discussed that a fifth deployment might be necessary to accommodate such an evolving scenario.

Using techniques discussed in the previous section effort is made to associate ONUs to OLTs from an initial condition where there is no specific bias for any particular ONU to be associated with any particular OLT; the bias is decided by the "cost" weight factor. The TDM underlay for the PON operation is taken as EPON. All OLTs are assumed to have a maximum downstream throughput capacity of 800 $\mathrm{Mb} / \mathrm{s}$ and a maximum upstream throughput capacity of $750 \mathrm{Mb} / \mathrm{s}$. The initial demand can be met with just three wavelength pairs, as the demand profile changes ONUs need to be re-allocated to meet the demand. In all the cases the upstream demand is not a limiting factor.

As the scenario evolves from Scenario 1 to Scenario 2, ONUs are reallocated such that the stated requirement is met. In this case again three wavelength pairs suffice to meet the demand. A total of 6 re-allocations take place as a result of change in user demands. Once the ONUs are re-allocated to other OLTs, the "cost" to associate them with any other OLT is doubled. This is to reduce the possibility of repeated re-allocations for ONUs which have already been re-allocated once. From Scenario 2 to 3 a total of 4 re-allocations take place. As the Scenario 4 evolves the fourth wavelength pair is required to meet the increased demand. A total of 17 reallocations take place from Scenario 3 to 4 . Figure 5-9 to Figure 5-12 illustrate the OLT to ONU allocation with the demand profile changing from Scenario 1 to Scenario 4 for a sample Network Group 1. If the network operator is constrained to not using the fourth wavelength pair for these set of 128 users, the bandwidth allocation to the ONUs is relaxed by the demand advertised. Figure 5-13 illustrates a 
case wherein all ONUs have advertised for 10\% relaxation. As the Scenario 4 evolves from Scenario 3, instead of using a fourth wavelength pair, the demand of ONUs is relaxed up to the advertised limit and the OLT-ONU associations are calculated; the figure shows the OLT-ONU associations for Network Group 3 in this scenario, without any relaxation and with $10 \%$ relaxation advertised. The number of ONUs associated with the OLTs for each of the scenarios is illustrated in Figure 5-14. Allocation by the OLTs in each of the scenarios in the downstream is illustrated in Figure 5-15. All optimization routines are solved with a commercially available optimization software; AIMMS with an XA14 solver. The load profiles are generated and analyzed with MATLAB ${ }^{\text {TM }}$ programs.

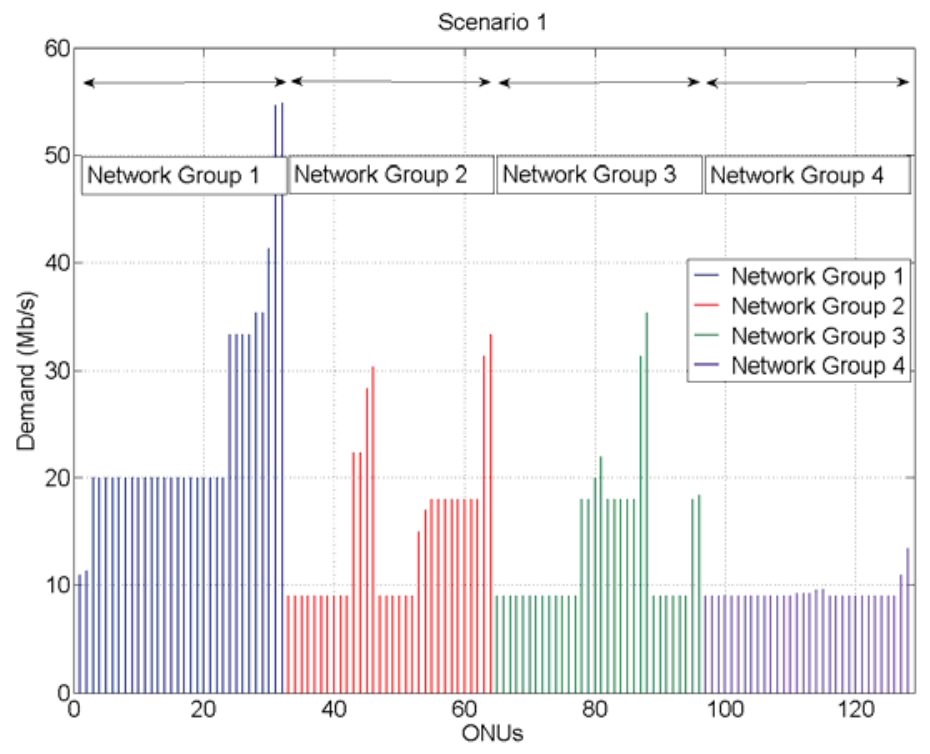

Figure 5-5: ONU demands in Scenario 1 


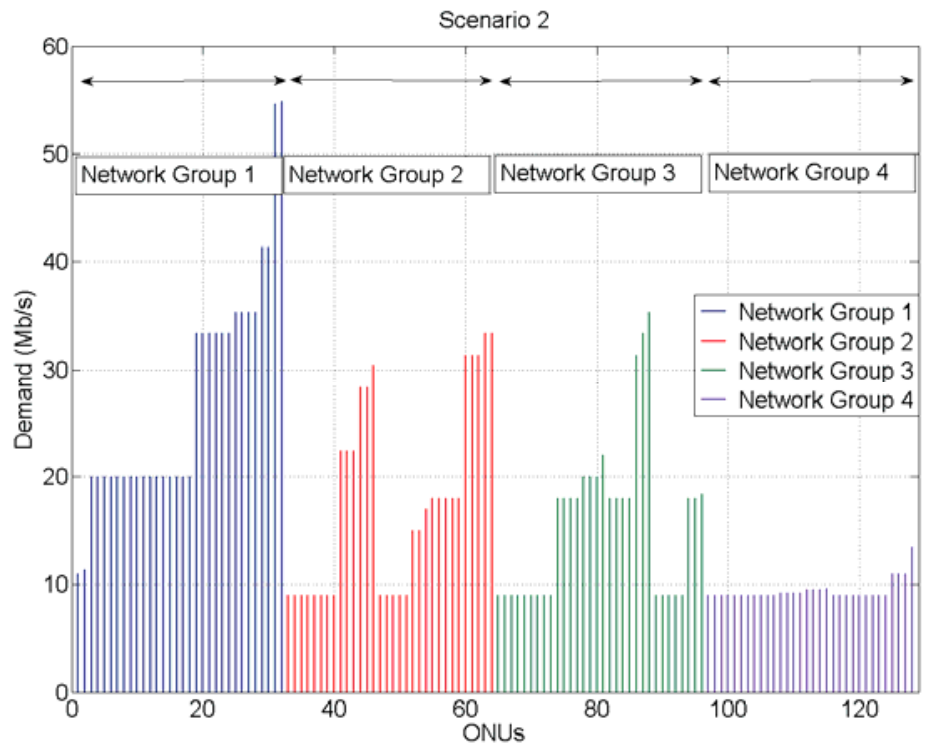

Figure 5-6: ONU demands in Scenario 2

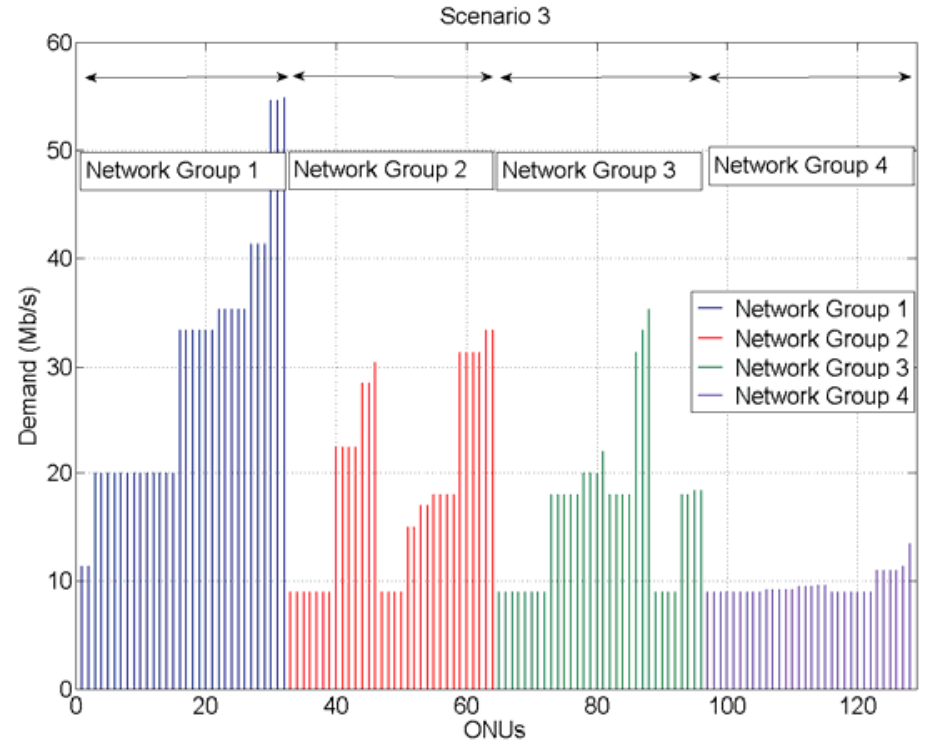

Figure 5-7: ONU demands in Scenario 3 


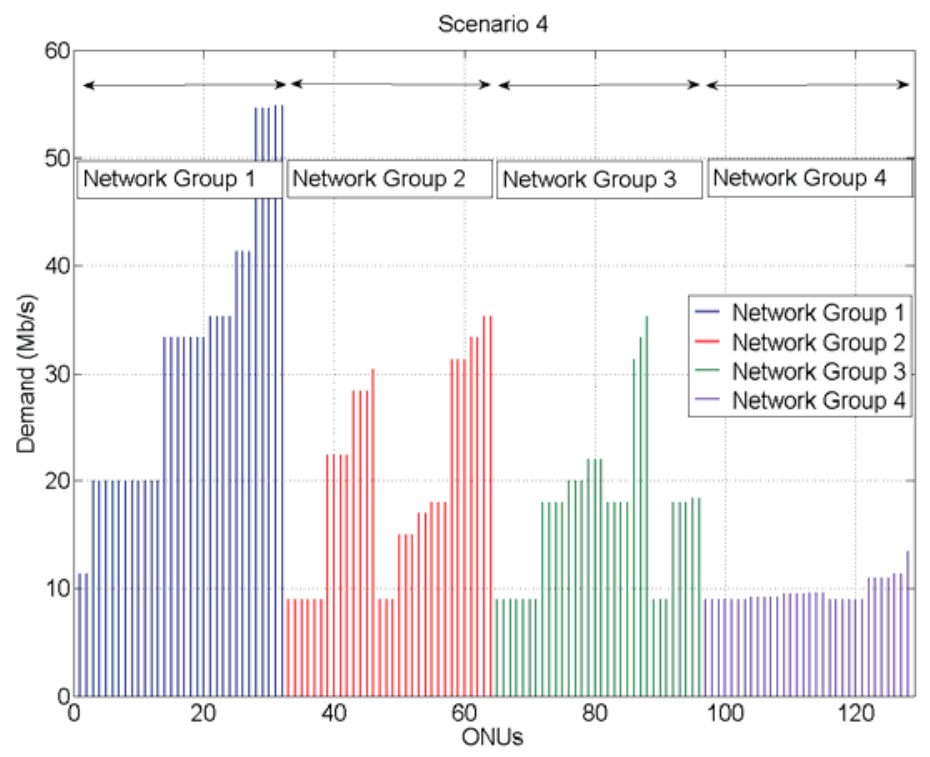

Figure 5-8: ONU demands in Scenario 4

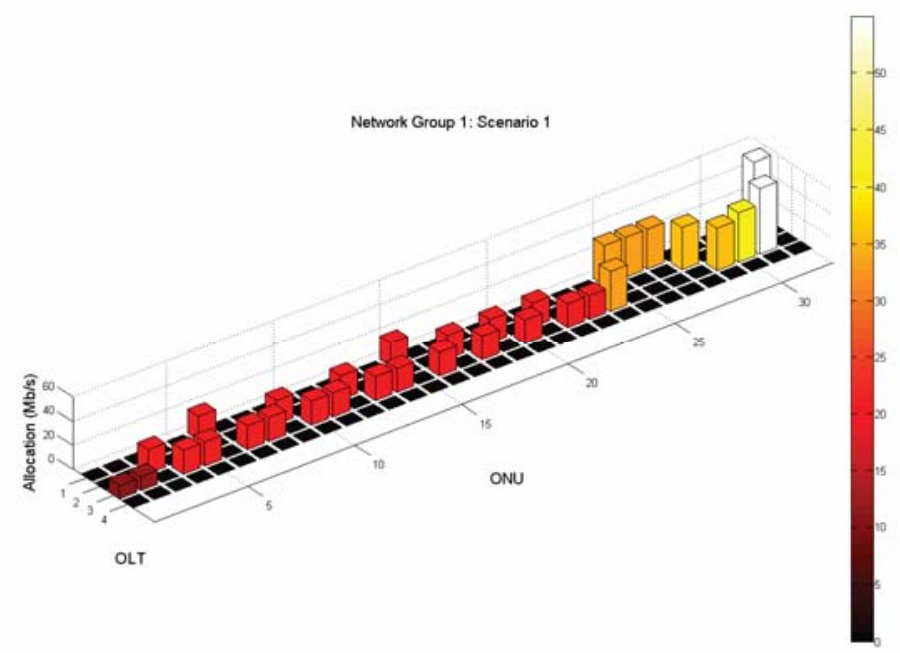

Figure 5-9: OLT-to-ONU association for Network Group 1 in Scenario1 


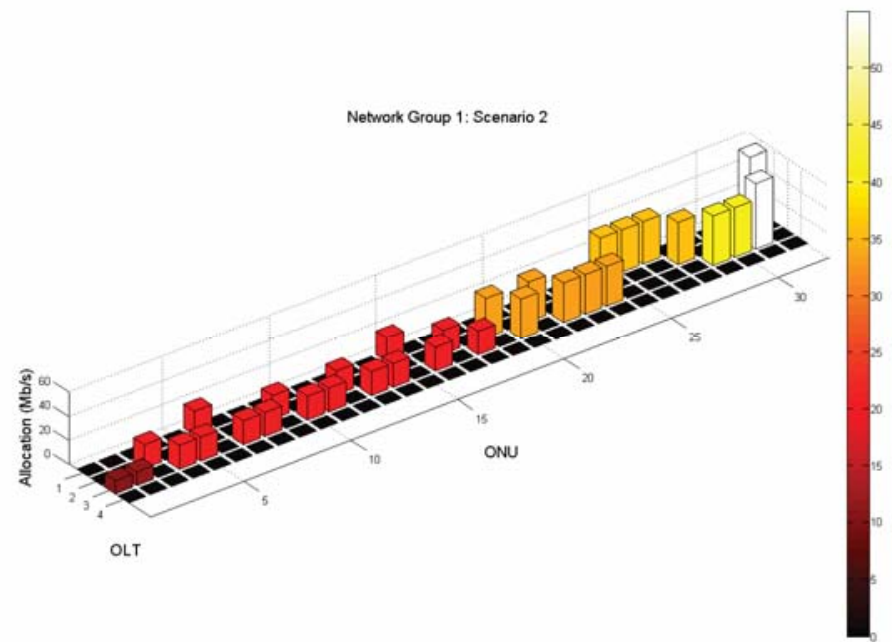

Figure 5-10: OLT-to-ONU association for Network Group 1 in Scenario 2

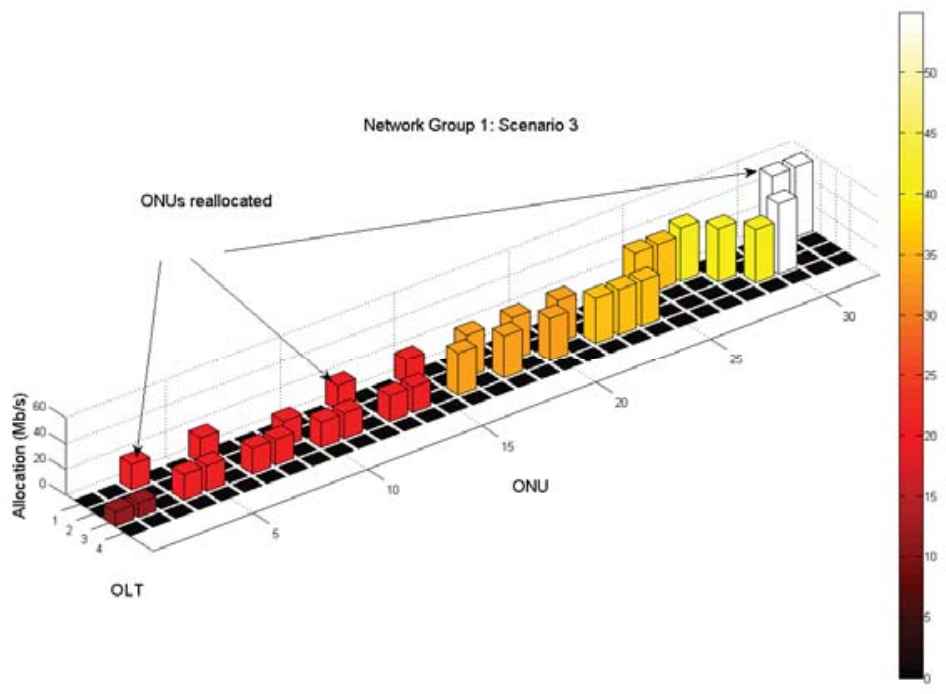

Figure 5-11: OLT-to-ONU association for Network Group 1 in Scenario 3 


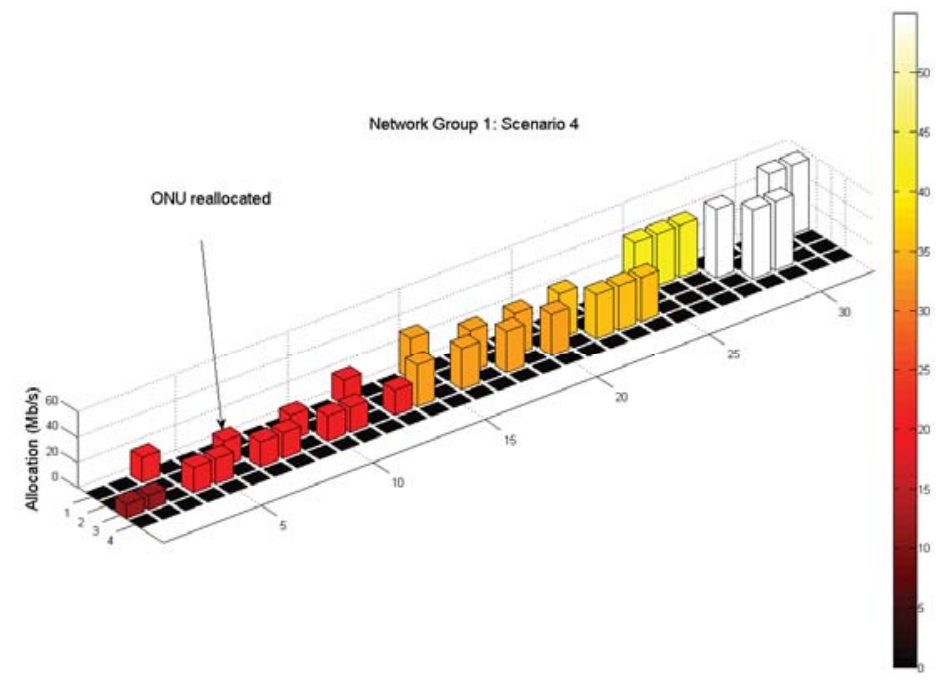

Figure 5-12: OLT-to-ONU association for Network Group 1 in Scenario 4

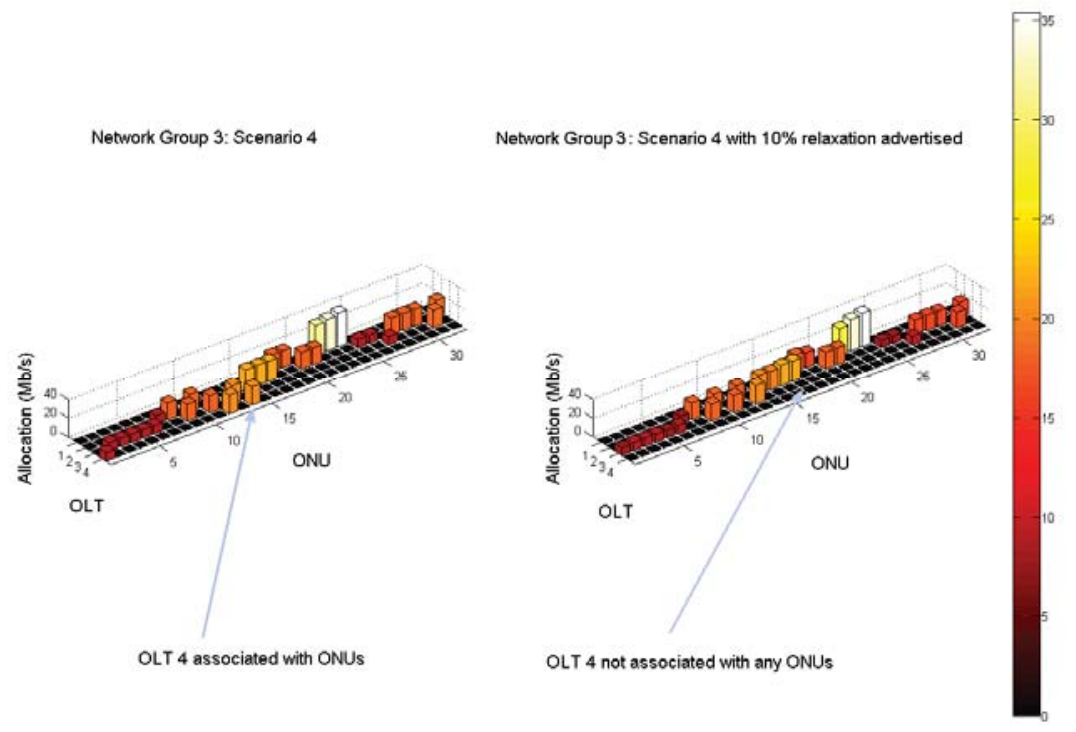

Figure 5-13: OLT-to-ONU association for ONUs in Network Group 3, without any and with $10 \%$ relaxation advertised by ONUs 


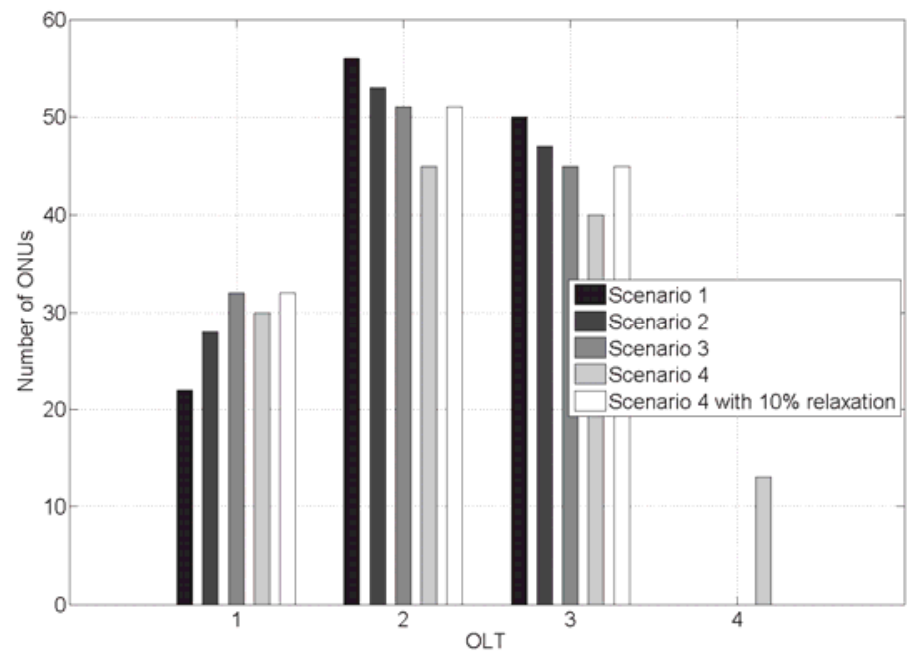

Figure 5-14: Number of ONUs associated with OLTs for different scenarios

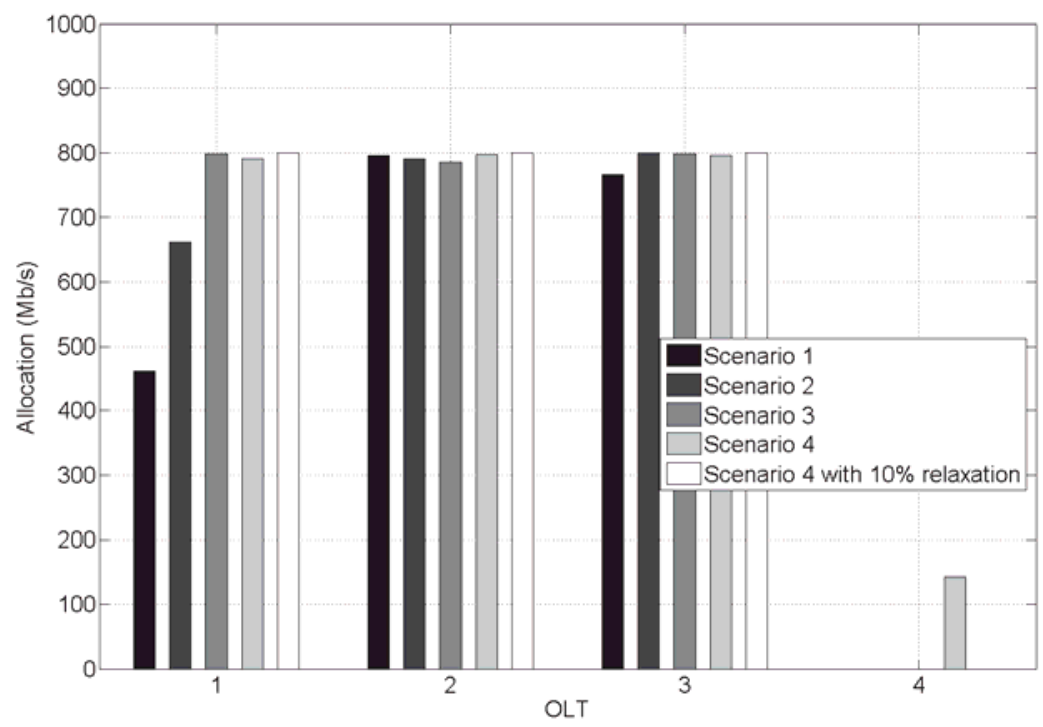

Figure 5-15: Allocation in downstream per OLT for different scenarios 
Typical hold times for media based services will be long during the presumed "busy hour" [60]. We try to analyze the network from a perspective of handling short duration high bandwidth requirements from additional new sources, for example if someone requires high bandwidth for download of files a few minutes. Two such cases are considered; in the first case new users require a chunk of $50 \mathrm{Mb} / \mathrm{s}$ for a finite duration during the period of interest, in the second case the requirement is for $100 \mathrm{Mb} / \mathrm{s}$ for a finite duration. It is also assumed that the fourth wavelength pair is now configured for use as a broadcast channel with 20 HDTV and 30 SDTV channel transmission, this allows for only $560 \mathrm{Mb} / \mathrm{s}$ free capacity in the downstream direction by potential new users. The bandwidth allocation to the incumbent ONUs is relaxed by a certain percentage and the optimization routine is used to seek additional space that can be created for new users.

\section{ONUs}

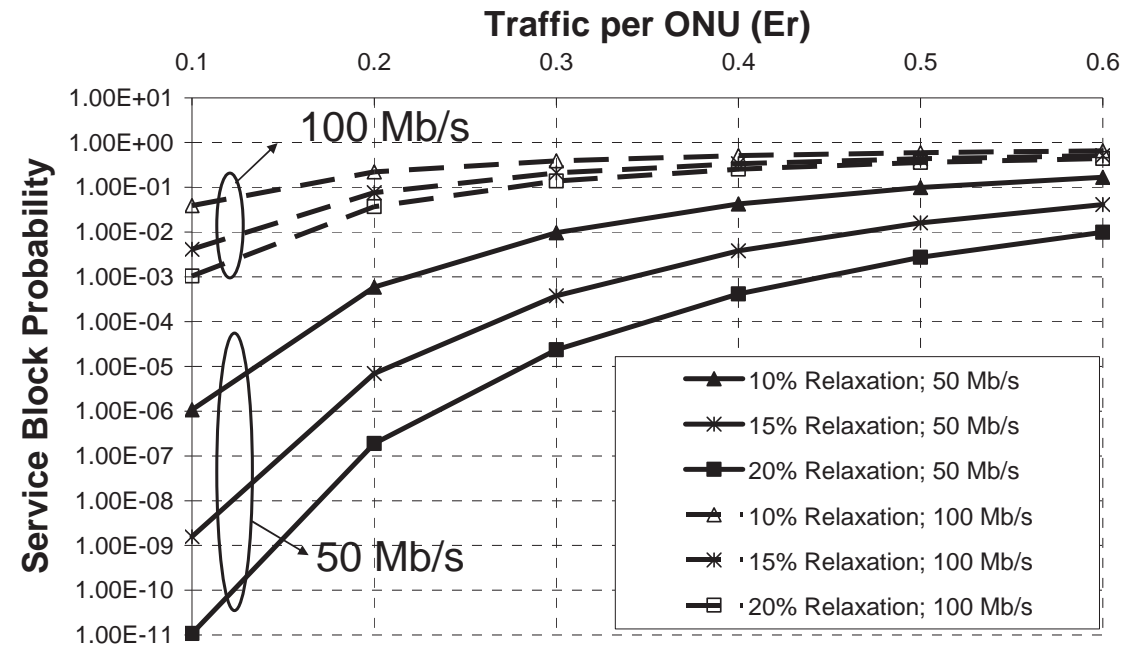

Figure 5-16: Blocking probability as a function of traffic generated (blocks of 50 and $100 \mathrm{Mb} / \mathrm{s}$ ) for $32 \mathrm{ONUs}$ 
The service requests and processing is modelled as an $\mathrm{M} / \mathrm{M} / \mathrm{m} / \mathrm{m} / \mathrm{s}$ queue [61], [Appendix B] where $\mathrm{m}$, is the number of such blocks of channels that can be accommodated in the network. The number of service requests contending for the available channels is $s$, for which the blocking probability is calculated [62]. Figure 5-16 illustrates the service blocking probability as a function of traffic generated per ONU for a case where only 32 new ONUs are requesting services. Figure 5-17 presents a different picture where the analysis presents the additional number of new ONUs that can be accommodated with a service blocking probability of $1 \%$, as a function of the traffic generated per ONU. The traffic generated per ONU is depicted in dimensionless units, Erlang (Er). This is the product of the call holding time and the call arrival rate during the period of interest.

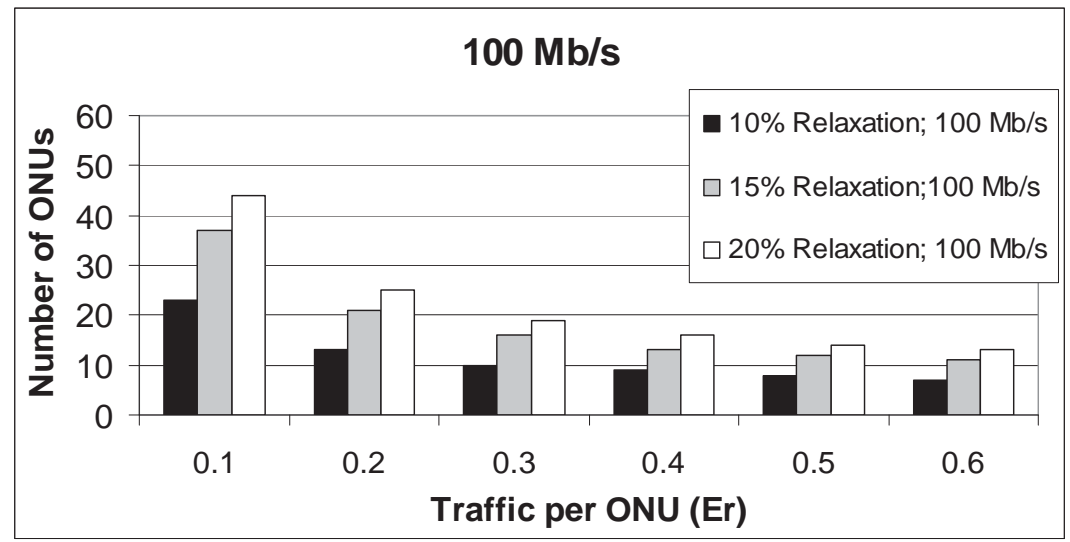

Figure 5-17: Number of additional ONUs that can be served by the network with a blocking probability of $1 \%$

The "perceived" bandwidth availability in a network is an important factor for customer satisfaction. In the use case demonstrated, for a network as a whole the average bandwidth availability per user (128 users with four logical PON deployments) is $25 \mathrm{Mb} / \mathrm{s}$. The range of peak bandwidth demands depending on the user profile varies from around $10 \mathrm{Mb} / \mathrm{s}$ to $55 \mathrm{Mb} / \mathrm{s}$. When all users agree to a relaxation of 
around $10 \%$, during the presumed "busy hour" an additional slice of $50 \mathrm{Mb} / \mathrm{s}$ in the downstream can be made available to up to 32 users with a service block probability of around $1 \%$ and $10 \%$ when the traffic demand per ONU is around $0.3 \mathrm{Er}$ and 0.6 Er respectively. A typical service block probability of between 1 and $10 \%$ is a commonly accepted figure in telco deployments for cellular and telephone networks during the busy hour. A heuristic argument can be drawn to show that the BBP like reconfigurable network can give a "perceived" bandwidth to up twice the peak demand assuming at any one time during the "busy hour" only $25 \%$ of the peak users temporally require more bandwidth with a traffic intensity of $0.3 \mathrm{Er}$ (assuming $1 \%$ service blocking probability).

\subsection{Summary}

This chapter presents the formulation to estimate an "optimal" network configuration for an optimal bandwidth distribution to the end user. The bandwidth to be allocated is a resource which has to be distributed given the constraints of the network. The constraints amongst others include the viability of associating an ONU to an OLT, the OLT being operational and when operational having enough capacity to service the need. A cost is associated with the bandwidth delivery given the constraints and an LP formulation is described which minimises this cost. The cost in the chapter is an abstract unit which would typically translate to the network opex for a telecom operator. The so called pricing model includes the aspect that a typical user would be ready to accept a lesser bandwidth delivery in return for an incentive of a lower price for bandwidth. This allows for freeing up more bandwidth in the network.

For the very first time the methodology is demonstrated for the use case discussed in the previous chapter. An evolving scenario is described where users require more bandwidth. The LP formulation described is used to allocate ONUs to OLTs such that the bandwidth delivery to all users is maximised. A BBP like reconfigurable network which is viewed as a "dynamic" switch can potentially allocate free band- 
width to existing users or to new users in case there is a temporal surge in the demand. A sensitivity analysis of the cost model is demonstrated where the freed up bandwidth is available for use to a typical user who might want additional bandwidth for a fixed temporal duration. It is clearly shown that a reconfigurable network needs disparity in terms of user profiles and demands to be able to free up resources, while this is the key to make bandwidth available, the network has to be such that this bandwidth can be allocated in a practical way where required. In a network the "perceived" bandwidth supply is an important aspect for customer satisfaction. This is the useable bandwidth that a user can get for short peak temporal requirements. It is demonstrated that with a reconfigurable BBP like network a user can "perceive" a bandwidth which is of up to twice the nominal peak bandwidth demand when around a quarter of the users in the network deployment seek additional bandwidth for short durations during the "busy hour". 


\section{Chapter 6}

\section{Service Delivery Aspects}

An access network provides a platform to facilitate services to the end user. Previous chapters have described in detail the use of bearer protocols which are in common commercial use. The BBP project considered the use of EPON based equipment to showcase the concept of a reconfigurable access network and this chapter presents the service delivery aspects in such a system.

\subsection{Ethernet Perspective of the Network}

The BBP network is an access network that stretches between the OLTs and ONUs; however, this network forms a part of the Ethernet access network that spans from the server of a service or content provider to the equipment such as a personal computer, voice-over-IP (VoIP) phones, digital TV, etc. in the end-user network. Figure 6-1 illustrates the "Ethernet" perspective of the network from the content/service provider to the end user. 


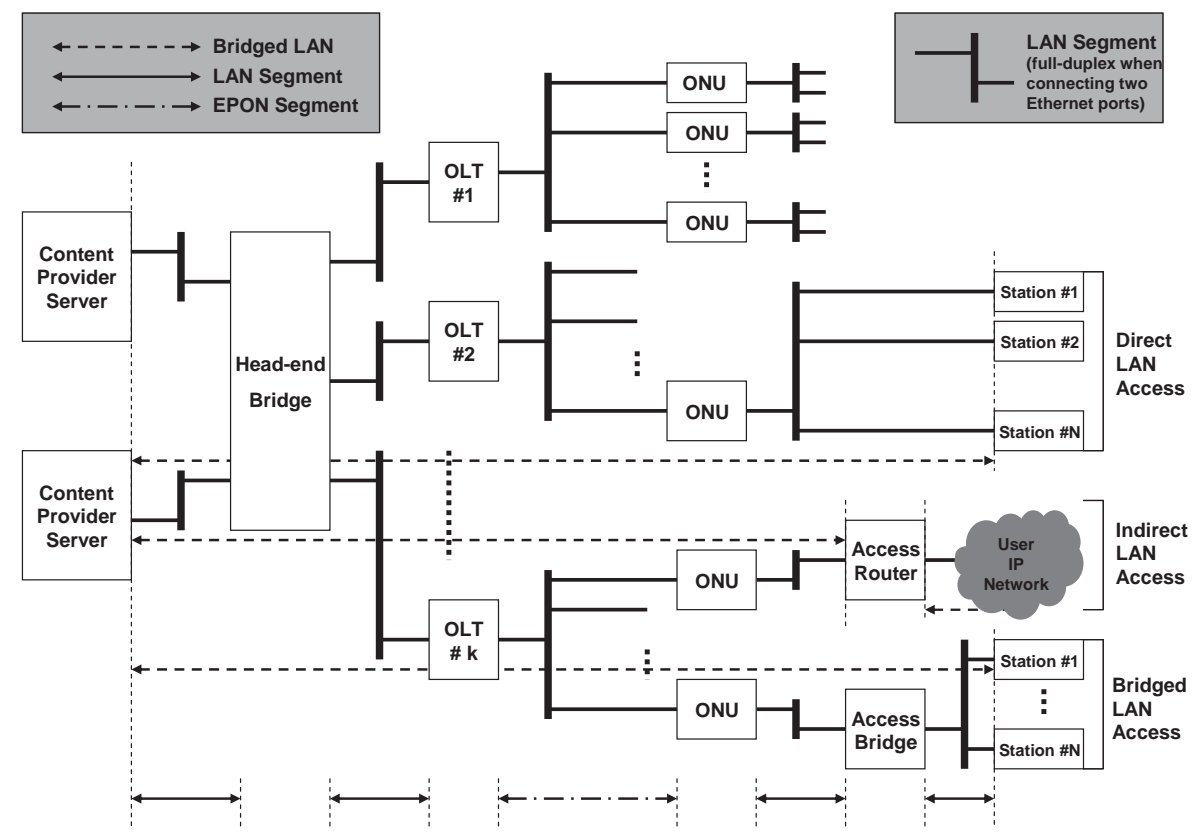

Figure 6-1: Ethernet perspective of the network

EPON operation is almost exactly like Ethernet operation but there are subtle differences. Forwarding in the EPON segment is different as done in a standard Ethernet LAN segment; instead of using the Destination Address (DA) field of the Ethernet frame for forwarding, the Logical Link Identifier (LLID) is used for forwarding. EPON is an emulation of a point-to-point connection in a point-tomultipoint scenario. The OLT maintains multiple MAC instantiations with each corresponding to an $\mathrm{ONU}$ associated with it (an additional MAC is maintained for broadcasts). The LLID forms a virtual port for the OLT to emulate a point-topoint service. The OLT learns MAC addresses of devices connected to the ONUs by processing the Source Address (SA) of inbound frames in the upstream direction. The OLT associates these MAC addresses to the LLIDs and in the downstream direction the OLT appends the LLID based on the Destination Address (DA) of the outbound frame. ONUs only "listen" to frames carrying LLIDs assigned to them (and to frames with the multicast mode-bit set to "1") and drop all other frames, 
irrespective of the MAC DA in the frame. The LLID/MAC association table is derived from the IEEE 802.1D "automatic learning" feature [63].

Service delivery in the access has transitioned from the traditional session based to client-less connection oriented delivery. This migration has meant that the traditional "best effort" services are no longer suitable for many newer services which are sensitive to packet loss, delay and delay variation. However, "best effort" services allow for significant over-subscription in the network, while real time applications like VoIP, VoD and gaming need committed bandwidth.
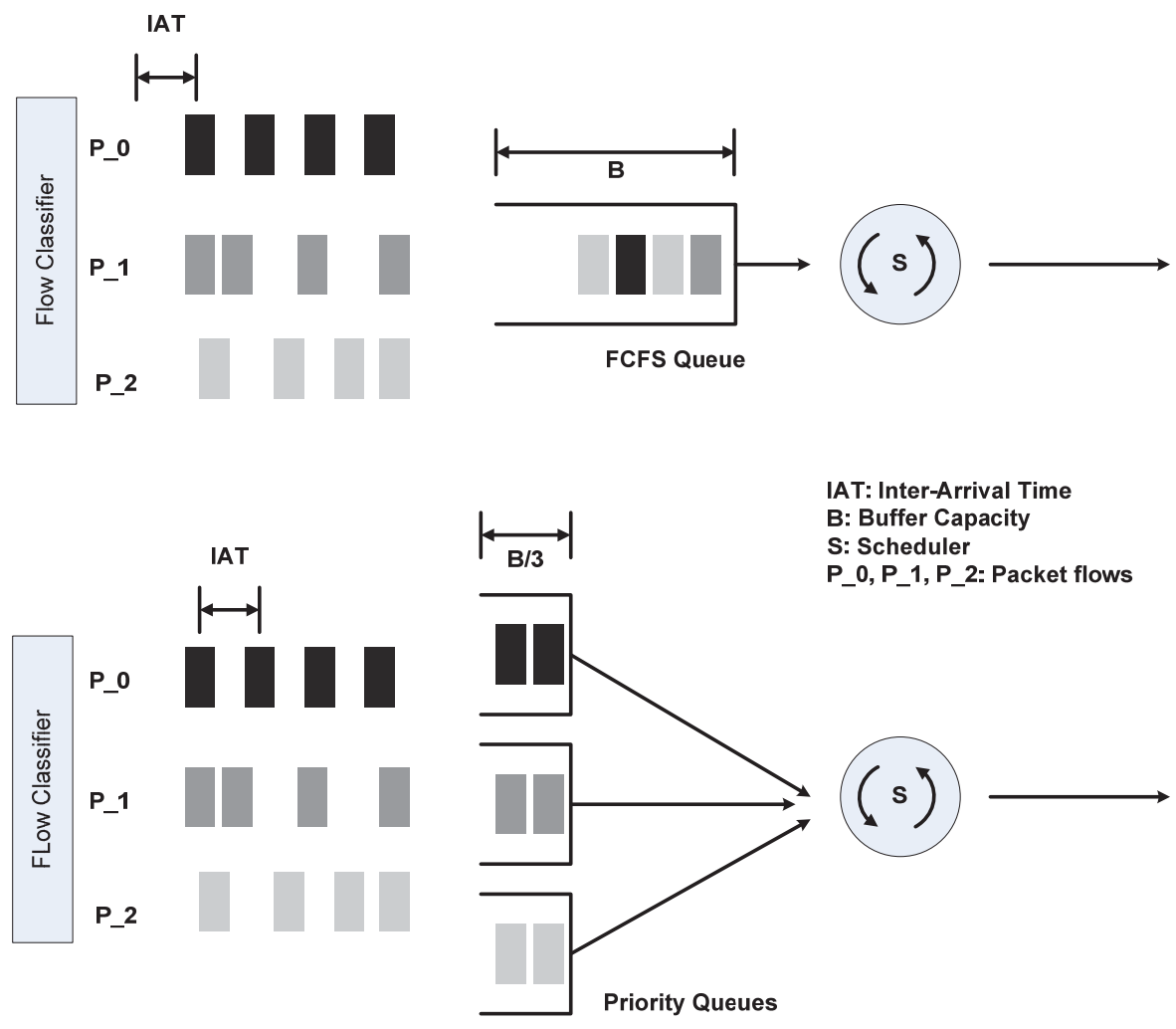

Figure 6-2: FCFS and Priority Queuing for scheduling 
Classification of traffic enables priority based scheduling algorithms to be implemented such that the delay and jitter are minimised while throughput is maximised for higher priority traffic. Congestion control and handling only become relevant when the congestion is a transient temporal phenomenon. During this period it is possible to allocate resources to optimise performance of time critical traffic. Typically in an access network there can be several instances of L2 switching elements and while these are not the determining factor, they remain a contributing factor to the overall network performance in terms of latency and jitter. The following model compares cases where there are three traffic flows; these are inbound on a switch subject to increasing loads and congestion duration. The traffic classes are handled with various queuing and scheduling algorithms to quantify the effects on performance during congestion periods. The traffic models three sources with a constant Inter Arrival Time (IAT) for the first source, and a log-normal IAT for the other two sources. Typical time sensitive traffic sources have a reasonably steady state IAT for long term periods, as for video or for short term periods as in VoIP during talk bursts [64]. Non real time traffic like HTTP is typically much more fragmented and noisier and the IAT can be modelled as a log-normal distribution [65].

Two queuing models are considered, the first being a First Come First Serve (FCFS) queue model with packets scheduled in order of arrival. The second queuing model incorporates Priority Queuing (PQ) where the scheduler dispatches packets according to a determined scheduling algorithm. The schematic of the two are illustrated in Figure 6-2. Three flow sources, $\mathrm{P} \_0, \mathrm{P} \_1$ and $\mathrm{P} \_2$ are considered with similar or dissimilar flow loads inbound to the switch. The scheduling algorithms considered with PQ are:

- Strict Priority: Packets are classified, queued separately and scheduled for dispatch in order of priorities $\left(\mathrm{P} \_0>\mathrm{P} \_1>\mathrm{P} \_\right.$2). A non empty higher order queue is always scheduled for dispatch earlier than that of a lower order priority. 
- Round Robin: Packets are classified, queued separately. The scheduler dispatches packets in a round robin fashion from non-empty queues.

- Weighted Round Robin: packets are classified, queued separately and scheduled for dispatch in a sequential manner similar to Round Robin but the number of dispatches per turn from a non-empty queue is in order of the weight allocated to the queue. Thus Round Robin is a special case of this with a weight of 1 allocated to all queues.

In all cases traditional congestion control policy is enforced with tail drop for a full buffer, packets once queued are not dropped. The following simulation results assume a total buffer capacity of 1000 frames. The duration of simulation represents the congestion duration. The overall load presented to the switch is a sum total of the load of the three flows (P_0, P_1 and P_2). In case of symmetric flows, the load presented by the respective flows is equal. For instance a total load of 2 presented to the switch consists of three flows each with a load of 0.67 (P_0=P_1=P_2=0.67). In case of asymmetric flows, the load of one of the flows is higher than the other two (taken as twice the value). For instance, now a total load of 2 could consist of $\mathrm{P} \_0=$ 1.00 with $P \_1=P \_2=0.50$. The load of a particular flow is created by varying the Inter Arrival Time (IAT) of the frames for the different flows. Hence a load of 1.00 for a flow would mean that the IAT is equal to 1.0 a.u while a load of 0.50 would mean that the IAT is 2.00 a.u and so on. The log-normal distributions have a standard deviation of 2.0. In the WRR scheme the weights used for scheduling $P_{-} 0$ is 2 and for $\mathrm{P} \_1$ and $\mathrm{P} \_2$ is 1 respectively. All simulations were modelled with Opnet Modeler ${ }^{\circledR}$. 

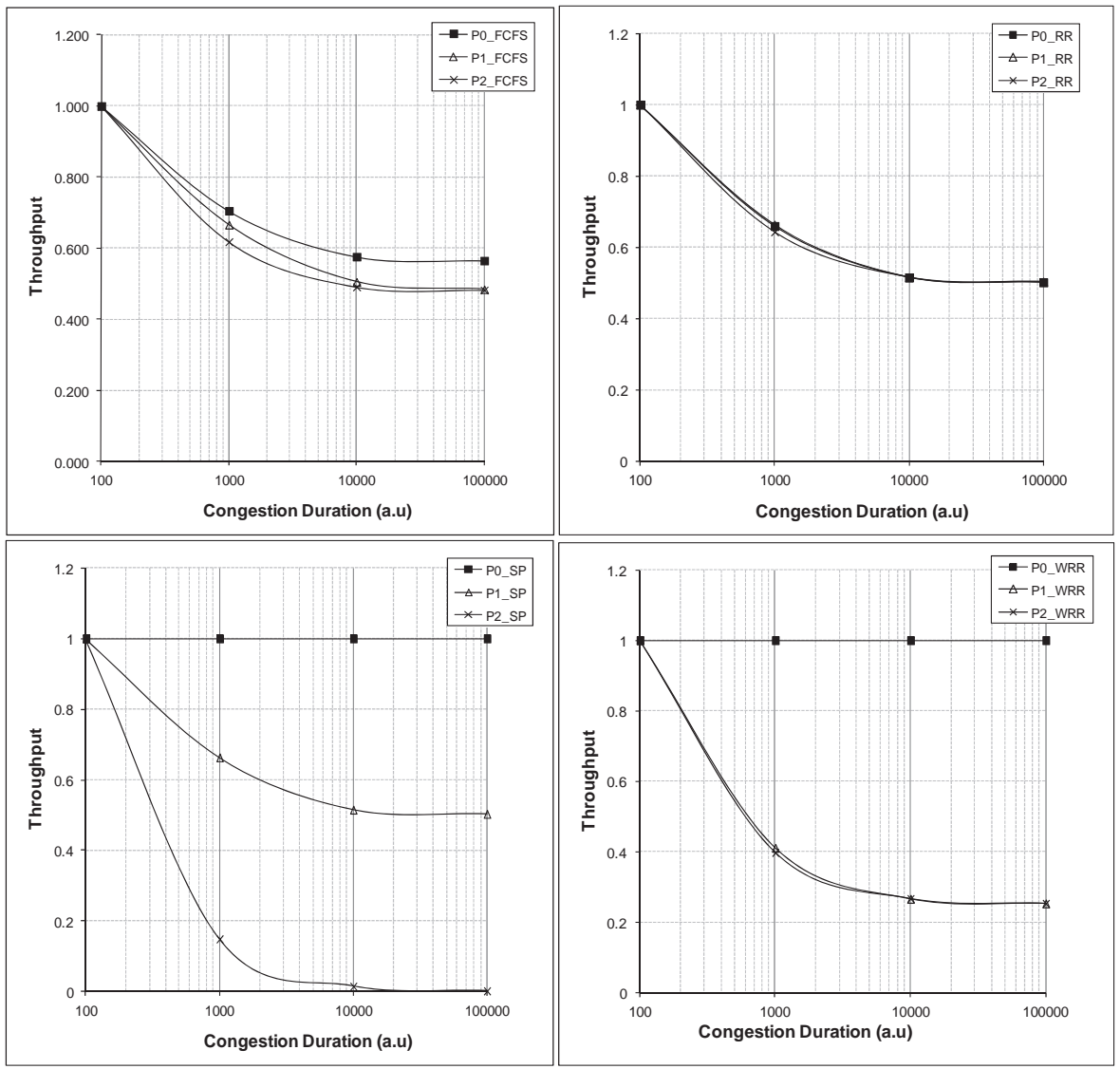

Figure 6-3: Throughput per flow with symmetric flows for FCFS, RR, SP and

WRR scheduling schemes (Total Switch Load=2.00, P_0=P_1=P_2=0.67)

Figure 6-3 illustrates the throughput achieved for the three symmetric flows when there is congestion (the overall load presented to the switch is 2 with each flow presenting a load of 0.67 respectively). In case of using an FCFS or RR scheme, the throughput asymptotically approaches an expected value of 0.5 . Since both these scheduling schemes do not prioritise any one flow, the high priority traffic $\left(\mathrm{P}_{-} 0\right)$ also suffers when there is congestion. To avoid this, the higher priority traffic can be given preferential treatment as is done with SP and WRR. In case of the SP, there is a clear hierarchy of scheduling. This can be detrimental to lower priority 
flows as they never get a chance to transmit as long as higher priority traffic is present.

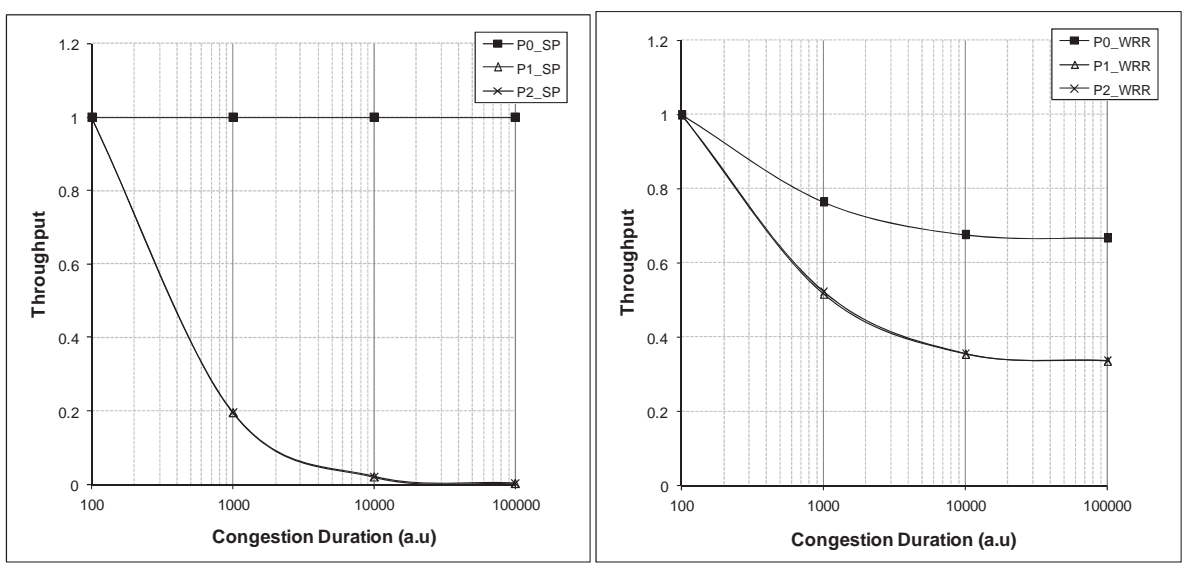

Figure 6-4: Throughput per flow with asymmetric flows for SP and WRR scheduling schemes (Total Switch Load=2.00, P_0=1.00, P_1=P_2=0.50). The throughput curves for P1 and P2 are overlapping for both scheduling schemes
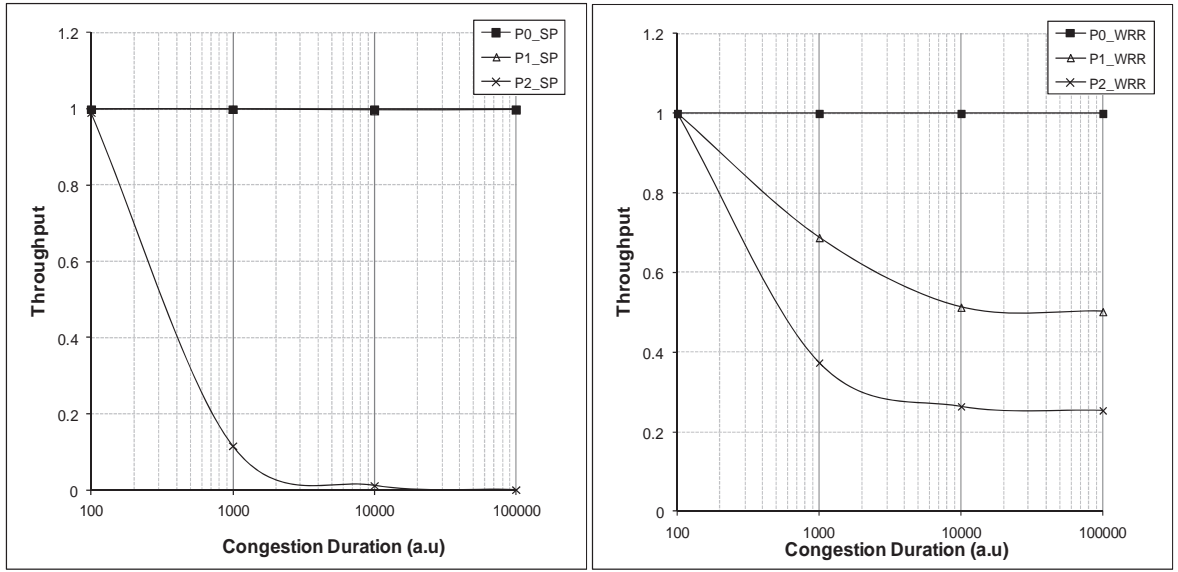

Figure 6-5: Throughput per flow with asymmetric flows for SP and WRR scheduling schemes (Total Switch Load=2.00, P_0=P_1=0.50, P_2=1.00). The throughput curves for $\mathrm{P} 0$ and $\mathrm{P} 1$ are overlapping for the $\mathrm{SP}$ scheduling scheme 
A WRR scheme presents a more balanced scheme of operation as here the flows can be allocated a share of time in the scheduling such that lower priority traffic is not swamped out by higher priority traffic. This is seen more clearly in Figure 6-4 where now while the overall load presented to the switch is the same as earlier (equal to 2.0), the highest priority flow (P_0) has twice the load compared to the other two flows. When a SP scheme is used, all the lower priority traffic (P_1 and P_2) is (the throughput curves for P1_SP and P2_SP flows are overlapping in the figure) dropped in preference to the higher priority traffic. Depending on the weights used, with a WRR scheme it is still possible to schedule lower priority traffic (the throughput curves for P1_WRR and P2_WRR flows are overlapping in the figure). Figure 6-5 illustrates the throughput when while having the same overall load (equal to 2.00), instead of the highest priority traffic having more load, the lowest priority traffic (P_2) has more load. With an SP scheduling scheme, the lowest priority traffic (P_2) is the most affected as it gets the least priority in scheduling (the throughput curves for P0_SP and P1_SP flows are overlapping in the figure). Once again a WRR scheme offers chance for the lower priority traffic to be scheduled during congestion periods.

Figure 6-6 illustrates the mean and maximum latency experienced by the different flows when the load presented to the switch is 1.00 (Note that while a linear scale is used for depicting the average latency, a logarithmic scale is used to depict the maximum latency). This represents a point where the switch starts to see congestion. Thus even though there are no packet drops in the flows at this point (throughput for all flows is 1), the latency of higher priority traffic can increase to unacceptable limits. The mean latency values illustrated are within $95 \%$ confidence intervals. For the P_0 flow (highest priority), with the application of SP or RR or WRR scheduling a delay bound can be ensured. In the first case for any packet, the maximum delay from the time it enters the buffer to the point it is transmitted, is up to 2 a.u. This occurs when there is another packet which is at the point of being processed for dispatch by the scheduler is followed by the current packet. In the case of RR and WRR scheme this can increase by the time required by the scheduler to dispatch 
packets from other flows. Here it is bound by a maximum of 3 a.u. as there are two other flows. To ensure that latency can be bound for a flow of traffic a scheduling scheme needs to be implemented (Note that the latency values for the WRR and the $\mathrm{RR}$ are coinciding in the graphs).

Figure 6-7 illustrates the packet delay variation for the different traffic flows when the offered load to the switch is 1.0. This parameter is often referred to as the jitter and represents the packet to packet delay. The delay variation of course depends on the type of traffic inbound but with a prioritised scheduling the delay variation can increase for lower priority traffic. However, what is important is to ensure that the delay variation is bounded for high priority traffic. 


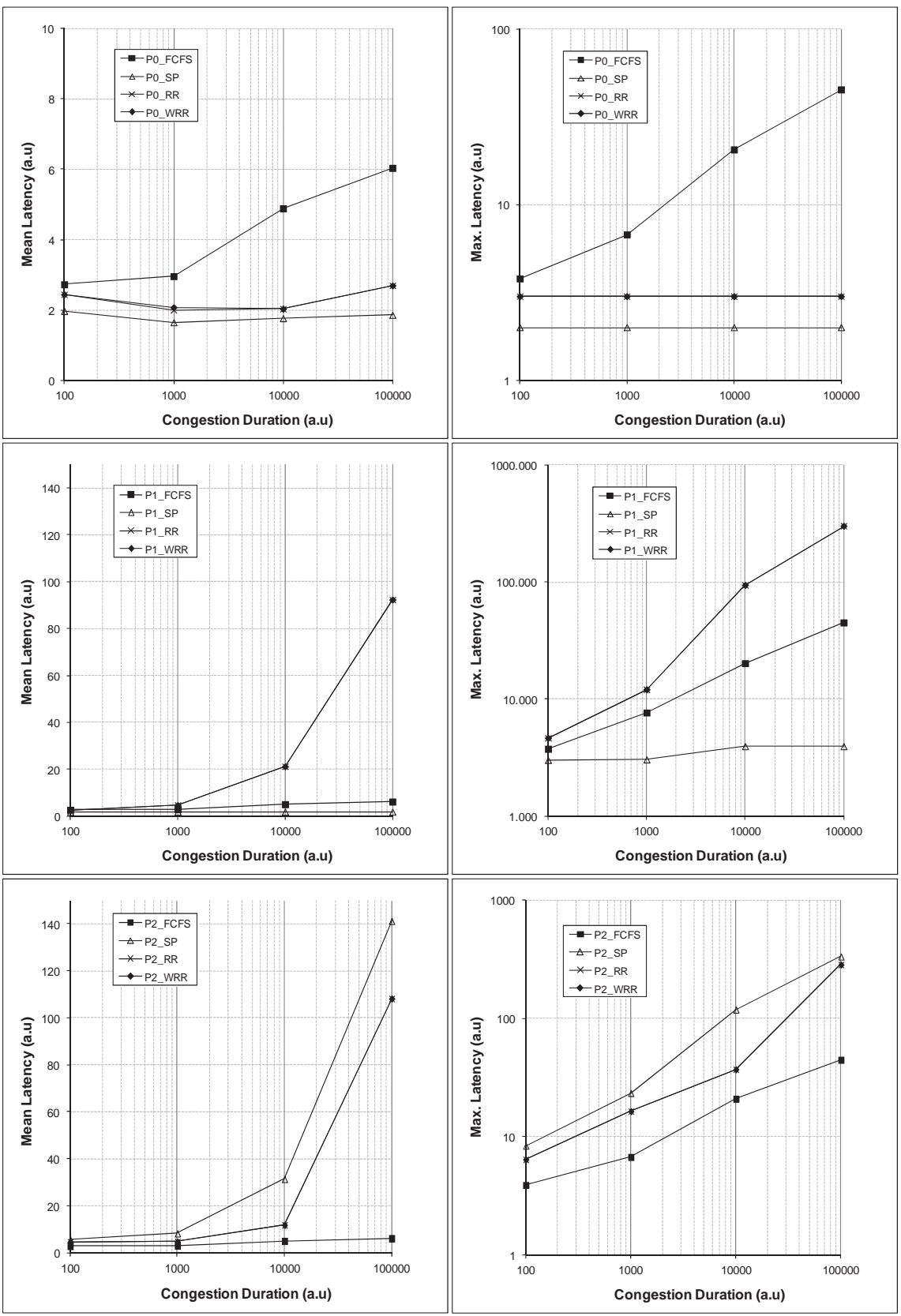

Figure 6-6: Mean and Maximum Delay for P_0, P_1 and P_2 for different scheduling schemes (Total Switch Load=1.00, P_0=P_1= P_2=0.33) 

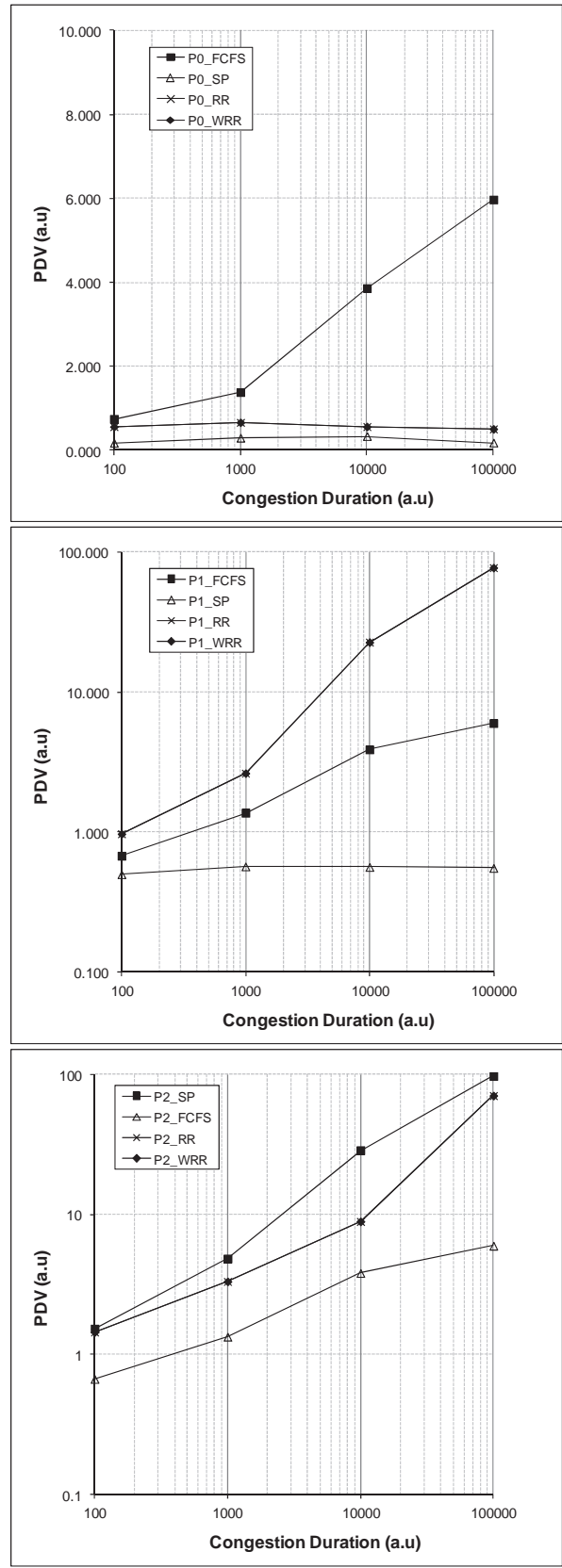

Figure 6-7: Delay variation for $\mathrm{P} \_0, \mathrm{P} \_1$ and $\mathrm{P} \_2$ for different scheduling schemes (Total Switch Load=1.00, P_0=P_1= P_2=0.33) 


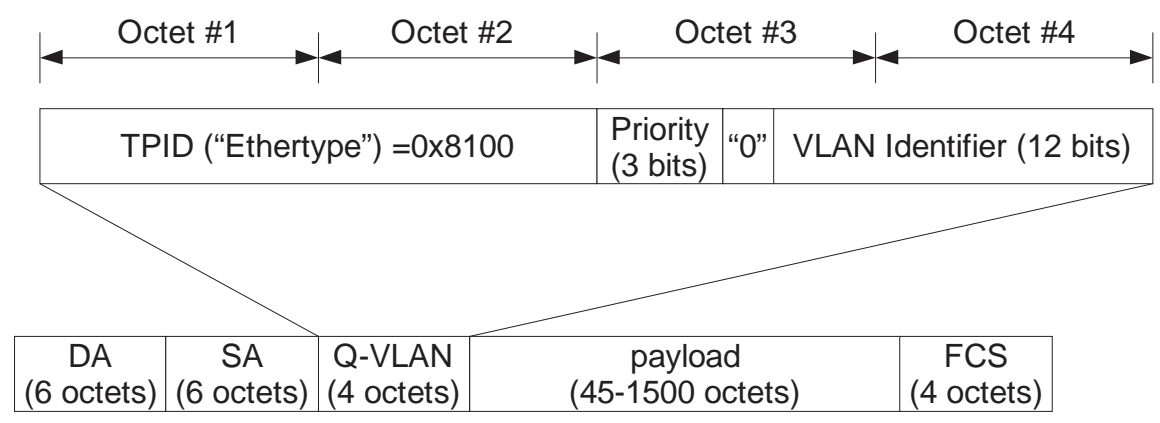

Figure 6-8: 802.1QVLAN tag

While traffic classification enables implementation of congestion management and scheduling algorithms to ensure QoS, it raises the question of whether this should be a layer 2 or a layer 3 solution. Since the access network is not a routing but essentially a switching network it is practical to use layer 2 technologies to distinguish traffic flows belonging to different end users and of different services over the same network. For Ethernet based services, the IEEE 802.Q[23] defines the VLAN tagging mechanism to do this. Figure 6-8 illustrates the layout of the VLAN tag and its position in the Ethernet frame. The priority bits in the VLAN tag allow for conveying priority information with tagged frames. Thus up to 8 classes of traffic can be distinguished based on these bits. Table 6-1 lists the categories of traffic defined and Table 6-2 lists the traffic class mapping based on the number of queues implemented.

The 12 bit VLAN-ID is enough to assign 4096 unique VLAN IDs (VIDs). $\mathrm{VID}=0 \mathrm{x} 000$ is reserved for tagged frames indicating only priority information while $\mathrm{VID}=0 \mathrm{xFFF}$ is a reserved value. $\mathrm{VID}=0 \mathrm{x} 001$ is not disallowed but is used as the default port VID of bridge ports. In effect 4094 values are available for use as unique VIDs. 
Table 6-1: Traffic typed defined in IEEE 802.1Q-2005

\begin{tabular}{lll}
\hline Priority & Acronym & Traffic Class \\
\hline 1 & BK & Background \\
0 (Default) & BE & Best Effort \\
2 & EE & Excellent Effort \\
3 & CA & Critical Applications \\
4 & VI & Video \\
5 & VO & Voice \\
6 & IC & Internetwork Control \\
7 & NC & Network Control \\
\hline
\end{tabular}

Table 6-2: Traffic type to traffic class mapping

\begin{tabular}{|c|c|c|c|c|c|c|c|c|}
\hline $\begin{array}{l}\text { Number of } \\
\text { Queues }\end{array}$ & BK & $\mathrm{BE}$ & $\mathrm{EE}$ & CA & VI & $\mathrm{VO}$ & IC & $\mathrm{NC}$ \\
\hline 1 & \multicolumn{8}{|l|}{$1^{\text {st }}$} \\
\hline 2 & \multicolumn{4}{|l|}{$1^{\text {st }}$} & \multicolumn{4}{|l|}{$2^{\text {nd }}$} \\
\hline 3 & \multicolumn{4}{|l|}{$1^{\text {st }}$} & $2^{\text {nd }}$ & & \multicolumn{2}{|l|}{$3^{\mathrm{rd}}$} \\
\hline 4 & \multicolumn{2}{|l|}{$1^{\text {st }}$} & \multicolumn{2}{|l|}{$2^{\text {nd }}$} & $3^{\text {rd }}$ & & \multicolumn{2}{|l|}{$4^{\text {th }}$} \\
\hline 5 & \multicolumn{2}{|l|}{$1^{\text {st }}$} & \multicolumn{2}{|l|}{$2^{\text {nd }}$} & \multicolumn{2}{|l|}{$3^{\mathrm{rd}}$} & $4^{\text {th }}$ & $5^{\text {th }}$ \\
\hline 6 & $1^{\text {st }}$ & $2^{\text {nd }}$ & \multicolumn{2}{|l|}{$3^{\text {rd }}$} & \multicolumn{2}{|l|}{$4^{\text {th }}$} & $5^{\text {th }}$ & $6^{\text {th }}$ \\
\hline 7 & $1^{\text {st }}$ & $2^{\text {nd }}$ & $3^{\text {rd }}$ & $4^{\text {th }}$ & \multicolumn{2}{|l|}{$5^{\text {th }}$} & $6^{\text {th }}$ & $7^{\text {th }}$ \\
\hline 8 & $1^{\text {st }}$ & $2^{\text {nd }}$ & $3^{\text {rd }}$ & $4^{\text {th }}$ & $5^{\text {th }}$ & $6^{\text {th }}$ & $7^{\text {th }}$ & $8^{\text {th }}$ \\
\hline
\end{tabular}




\subsection{Service Architecture}

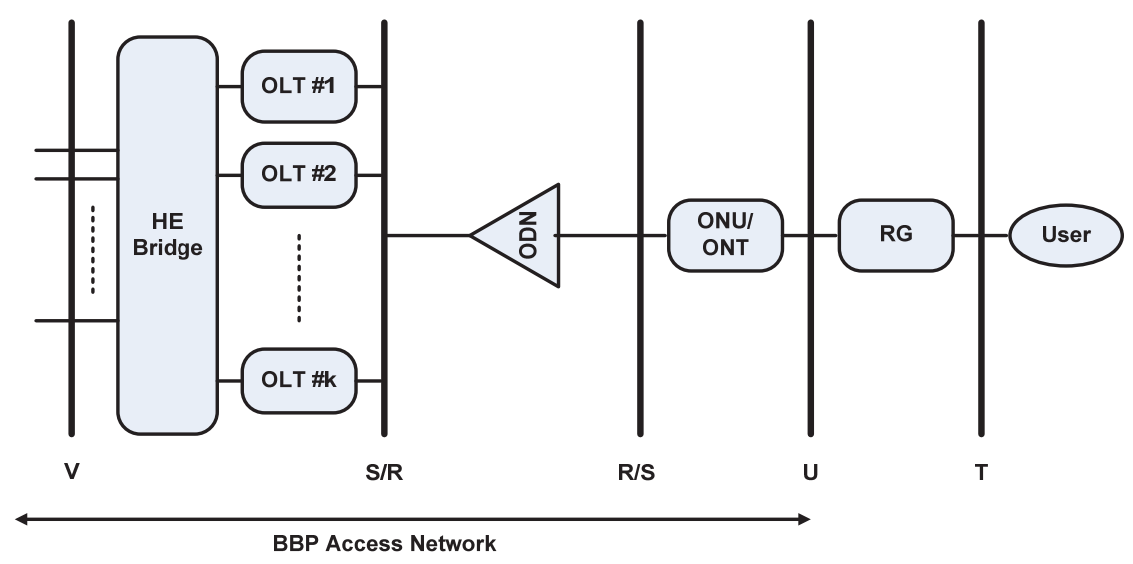

Figure 6-9: Network architecture with reference points

The TR-101 [66] forms the de-facto industry standard to dimension service delivery architecture for access networks. The technical report was stated for migration of ATM based aggregation networks to Ethernet based aggregation networks in the DSL framework and forms the platform to dimension service delivery in other technologies like Ethernet based service provision in GPON access networks [67] and for similar provision in EPON access networks (work was being done as part of WT-200, at the time of writing the text, the technical report has since then been released as [68]). This framework is used to describe the overall service architecture of the BBP network. Figure 6-9 illustrates the reference points for the BBP network. The $\mathrm{V}$ interface defines the boundary between the Access and Regional/Metro networks. The Optical Distribution Network (ODN) including the $\mathrm{RNs}$ stretch between the $\mathrm{S} / \mathrm{R}$ and $\mathrm{R} / \mathrm{S}$ reference points. The $\mathrm{U}$ interface demarcates the termination of the access network and the customer network. The user interface is designated with the $\mathrm{T}$ reference point. The reconfigurable access network stretches between the $\mathrm{V}$ and $\mathrm{U}$ reference points. 
GPON distinguishes between an Optical Network Unit (ONU) and an Optical Network Termination (ONT). The ONU is defined for FTTB/C applications with POTS/xDSL interfaces towards the end customer, while the ONT is defined for FTTH applications with Ethernet/MoCA interfaces towards the end customer. In this configuration the Residential Gateway (RG) might or might not be integrated with the equipment. EPON on the other hand refers only to ONU which is understood to be in the subscriber premises. In the context of definition herein we refer to the ONU as being equivalent to the GPON definition of ONT with a single Gigabit Ethernet interface for access only and multiple Gigabit Ethernet interfaces for aggregation and access towards the end customer. Figure 6-10 which is adapted from TR-101 illustrates the service access points for reference in this network illustrating an ONT with an external RG and an ONT with integrated RG. The ONT can support multiple RG interfaces (not illustrated). In all cases the $\mathrm{U}$ interface is a Gigabit Ethernet interface which could be a 1000BASE-T or 1000BASE-X interface.

As discussed earlier, use of VLANs allows for classification of traffic within the scope of the network. Two distinct architectural models are possible for use of VLANs.

- 1:1 Model or the Customer VLAN model

- $\quad \mathrm{N}: 1$ Model or the Service VLAN model 

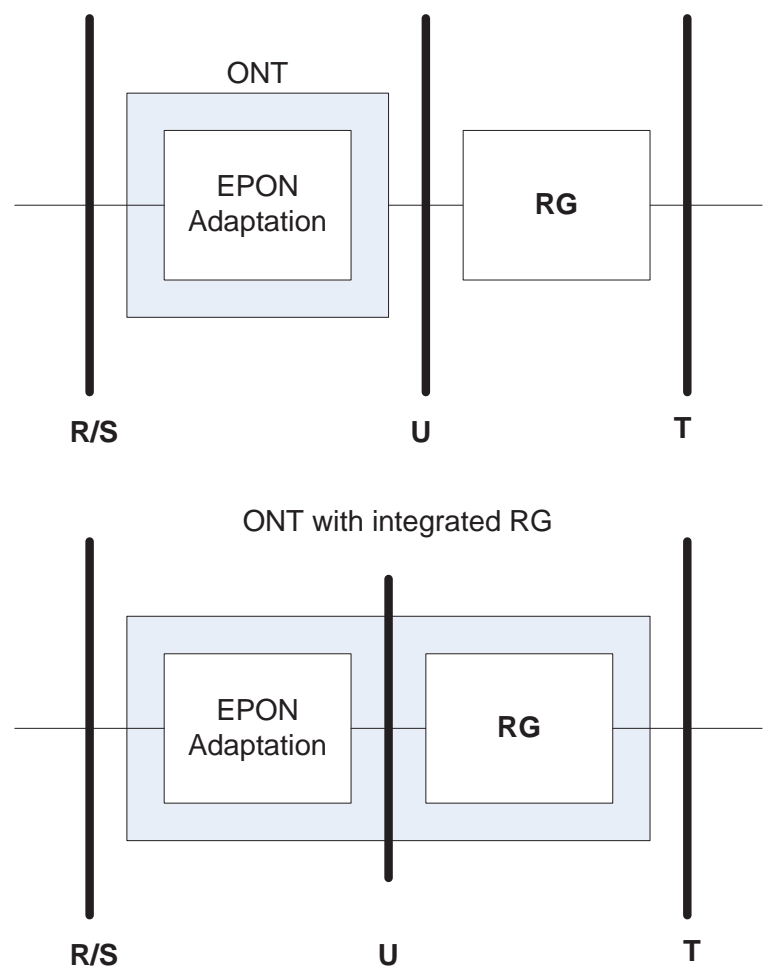

Figure 6-10: ONU without and with integrated Residential Gateway

Figure 6-11 and Figure 6-12 illustrate the logical connectivity for the Customer VLAN and Service VLAN cases respectively. Both architectures have their strong points and drawbacks [69]. One of the key parameters to decide is whether the service dimensioning is more oriented for broadcast/multicast or unicast. In a customer VLAN model, each customer and consequently all the services per customer can be monitored at the head-end. This enables better dimensioning of the service modelling deeper in the network (typically at the Broadband Service Aggregator or BSA). This kind of architecture on the other hand replicates all multicast and broadcast traffic across all the Customer VLANs. 


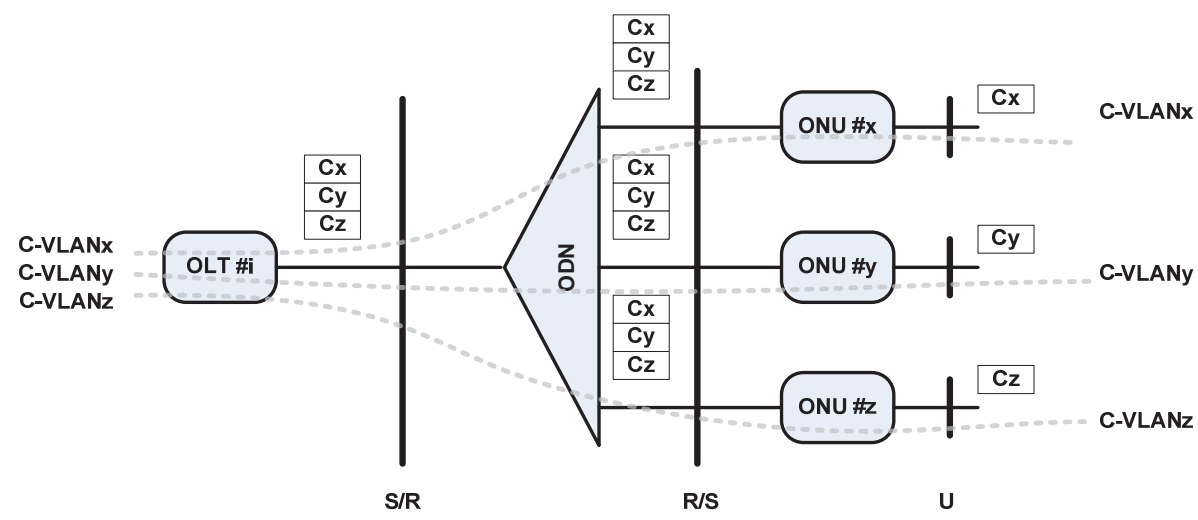

Figure 6-11: Customer VLAN

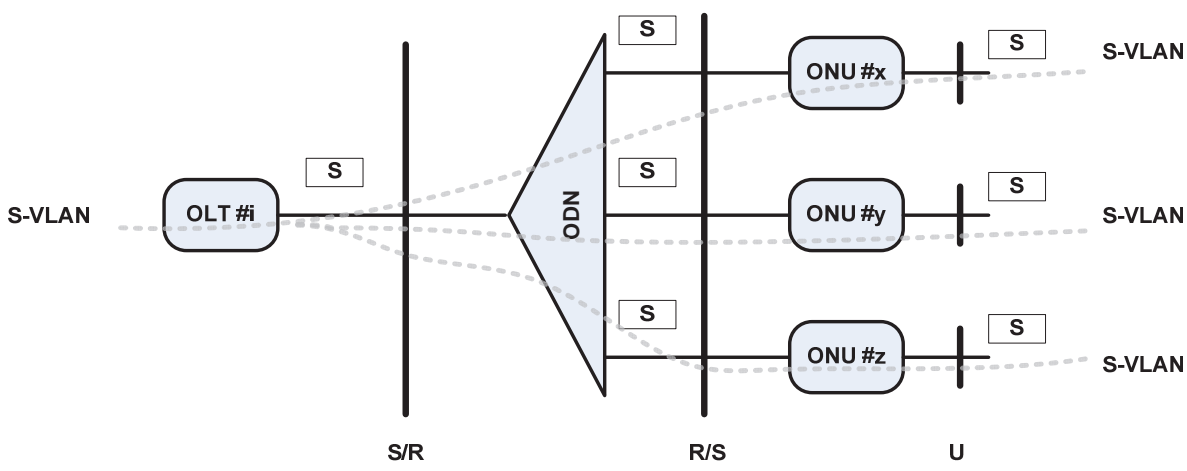

Figure 6-12: Service VLAN

In a PON even though all ONUs have access to the traffic, each ONU will be accessing a different VLAN and thus bandwidth is wasted because of the replication of traffic. In a Service VLAN model on the other hand this would not be the case, however, it is computationally intensive to determine the bandwidth flow per ONU and hence to determine actual customer use. In a dynamically reconfigurable network, the key element is to determine the bandwidth required per user hence the Customer VLAN model is more suitable. 
Since the number of unique VIDs that can be issued are limited, it is important to dimension VLAN usage. The access network can be used to deliver:

- Ethernet network access (with a typical IP over Ethernet stack)

- Provide transparent LAN services (TLS).

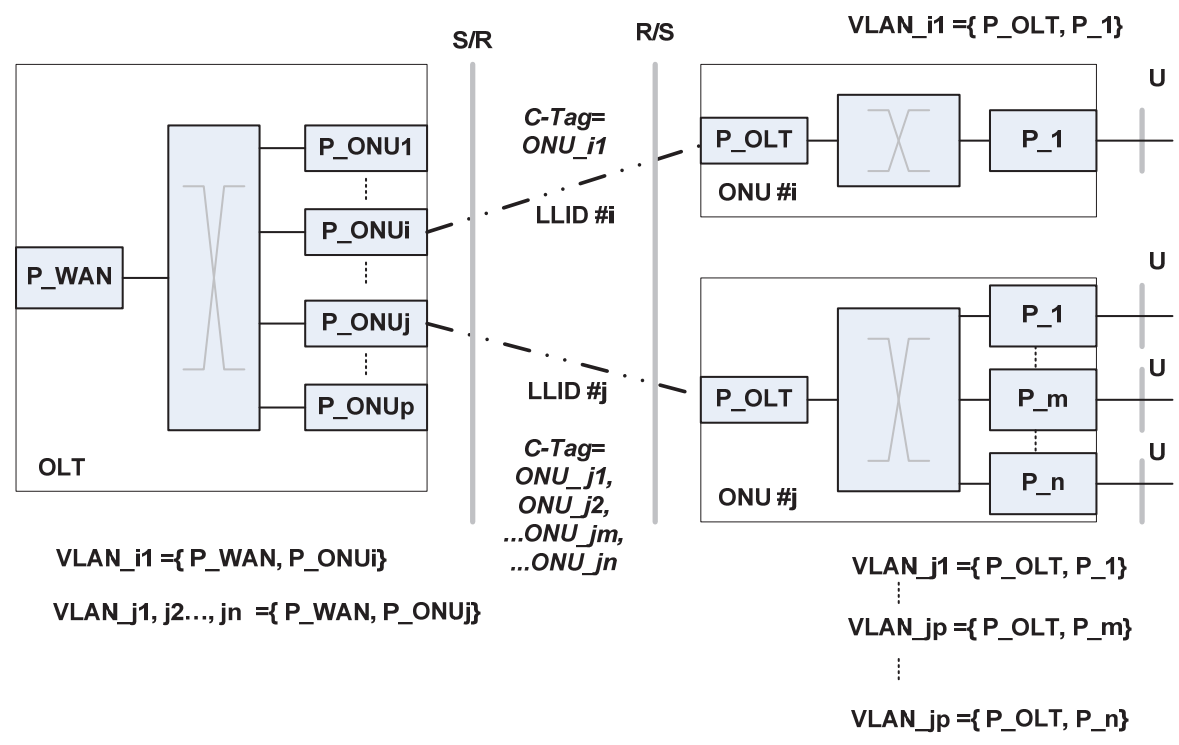

Figure 6-13: C-Tags for single and multi-subscriber ONUs for Ethernet access

In case of providing Ethernet network access we need to consider whether the ONU is used for a single end subscriber or for multiple end subscribers. For a single subscriber tagging of frames for the purpose of classification only is sufficient. The traffic between the OLT and ONU is already a logical point-to-point transmission and using VLAN-IDs to identify traffic flow does not add value. The traffic flow per subscriber can be controlled by the OLT in the downstream direction by varying the egress policy and in the upstream direction by the DBA policy. The OLT can do a simple LLID to VLAN translation and add a Q- Tag (C-Tag) for transmission towards the $\mathrm{HE}$ Bridge and further. If the ONU has multiple subscriber interfaces, use of VLANs enables identification and control of traffic flow in both directions on 
a per subscriber interface basis. Use of Q-Tags with a VID for all subscriber interfaces on ONUs makes implementation more uniform (Q-Tags are the VLAN tags as defined in [23], these are defined with a TPID $=0 \times 8100$ ). Figure 6-13 illustrates the use of VLANs for logical separation of traffic flow from subscribers towards the WAN interface. At the OLT there are multiple virtual port instantiations which are providing a point-to-point logical link with the ONUs. The OLT-ONU traffic is tagged with a Q-Tag (C-Tag), denoted by ONU_yz, where y is the running index for the ONU and $z$ is the running index for the subscriber number per ONU. As mentioned earlier, while tagging is required for classification of traffic, for a single subscriber a VID does not give added information as the OLT can add/remove the tag based on LLID to VID translation. However, for a multi-subscriber ONU, assigning VIDs is required to identify traffic flows to individual subscribers. The VID tag has to be unique and can take any of the valid numbers from 1 to 4094 . The port mapping for VLANs is also illustrated.

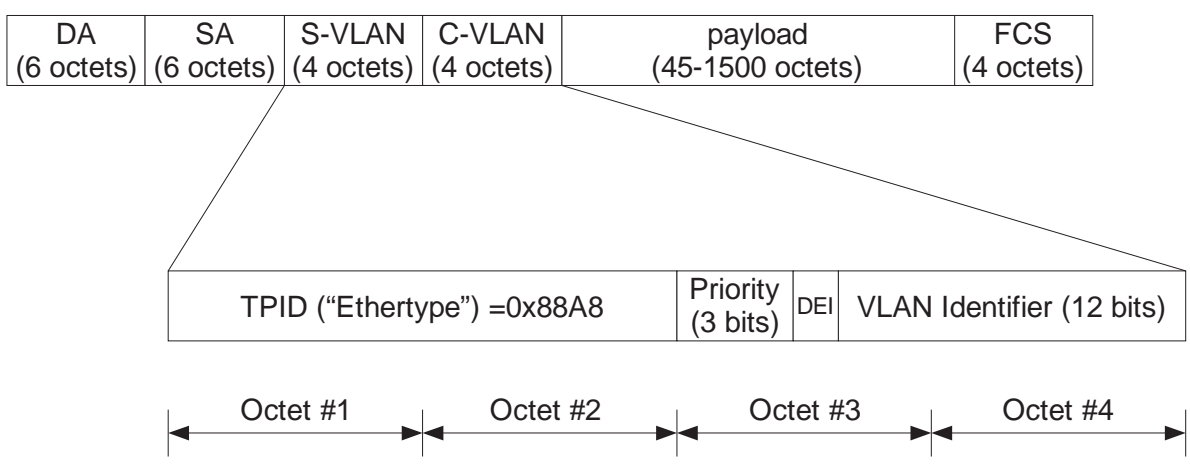

Figure 6-14: 802.1ad VLAN Tag

Providing TLS in an access network is required typically for business customers. This service provides connectivity between two or more subscriber sites. The IEEE 802.1 ad-2005 amendment to the 802.1Q-2005 standards provides for TLS services through provider bridging using stacked VLANs or Q-in-Q. An additional "service" 
VLAN tag or S-Tag is added to any existing "customer" tag or C-Tag (this is the same as the Q-Tag referred to earlier, the S-Tag used to have a non standardized usage of TPID $=0 \times 9100$, this has been standardised to a TPID $=0 \times 88$ A8). Figure 6-14 illustrates the placement of the S-Tag in an Ethernet frame. Functionally the OLT treats the frame in a similar way except that now the frames are either double tagged (if there is a C-Tag present) or single tagged with an S-Tag (if there is no CTag). The use of double tagging should not be confused with the earlier reference for VLAN architectures (Customer and Service VLANs).

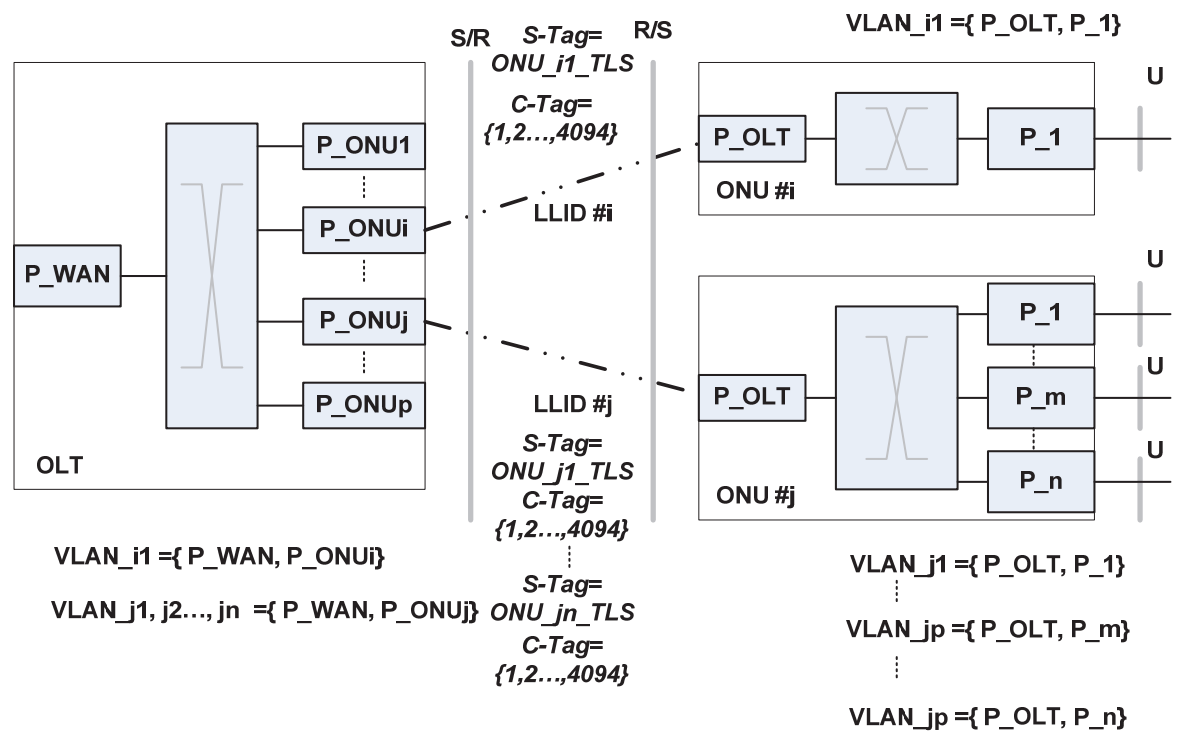

Figure 6-15: S-Tags for single and multi-subscriber ONUs for TLS

Figure 6-15 illustrates the use of double tagging for providing TLS. The OLTONU traffic is tagged with an S-Tag, denoted by ONU_yz_TLS. Where $y$ is the running index for the ONU and the $\mathrm{z}$ is the running index for the TLS service to an ONU. The frames themselves might be double tagged with an additional C-Tag which is reserved for use within the client network or single tagged with just the STag where no C-Tags are used in the client network. Like the earlier case, the STag has to be unique and can take any of the valid numbers from 1 to 4094 . The 
essential difference here is the use of an S-Tag instead of a C-Tag (or Q-Tag as referred to earlier) to distinguish traffic flows. This also means that the S-Tag allocated has to be unique not only within the logical PON supported by a single OLT but by the entire reconfigurable access network.

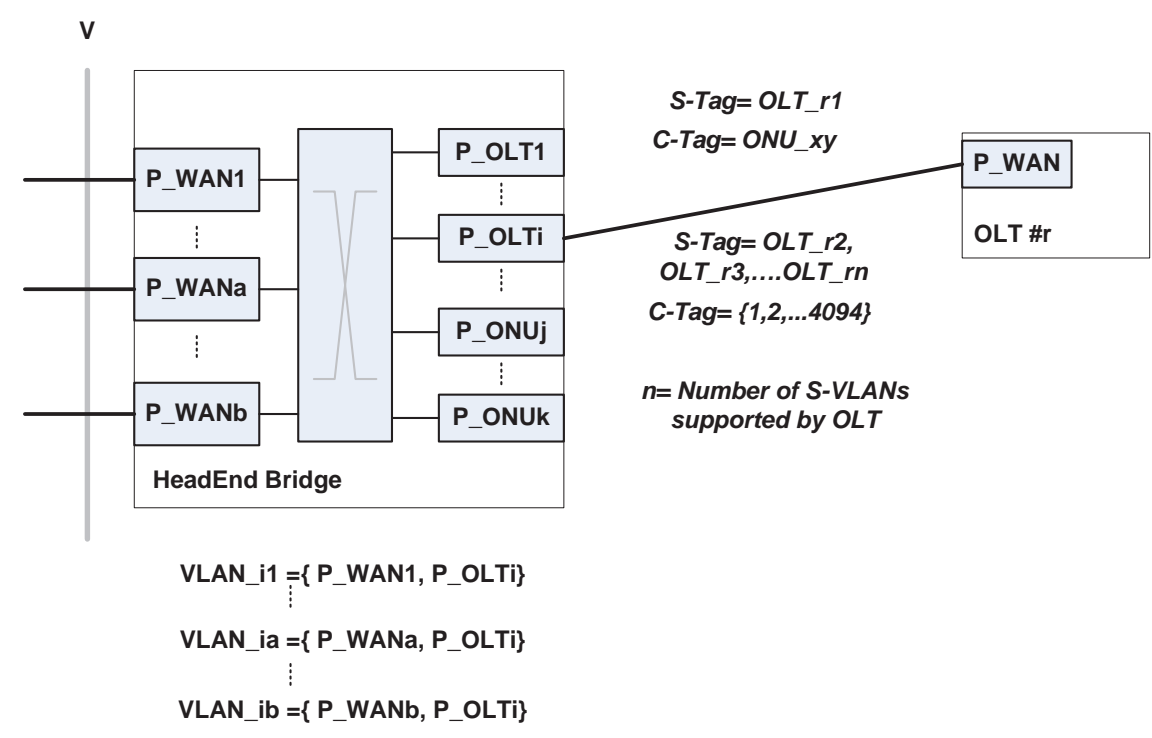

Figure 6-16: S-Tags for traffic flow upstream from OLT towards HE Switch

Figure 6-16 illustrates the traffic distinction towards the HE Switch and further from an OLT. The traffic flow between the HE Switch and OLTs is distinguished by S-Tags. This is denoted by OLT_rs where $r$ is the running index for the OLT number and $\mathrm{s}$ is the running index for the S-Tags supported by the OLT. Thus all Ethernet Network Access traffic with Q-Tags (S-Tags) are double tagged retaining the original C-Tag for distinguishing individual subscriber traffic flows. All TLS service instances are transparently mapped such that all service instances terminating at the HE switch have unique S-Tags. The HE switch transparently transmits the S-Tag for further processing deeper in the network where traffic flows per subscriber can be related to traffic flow per service. 


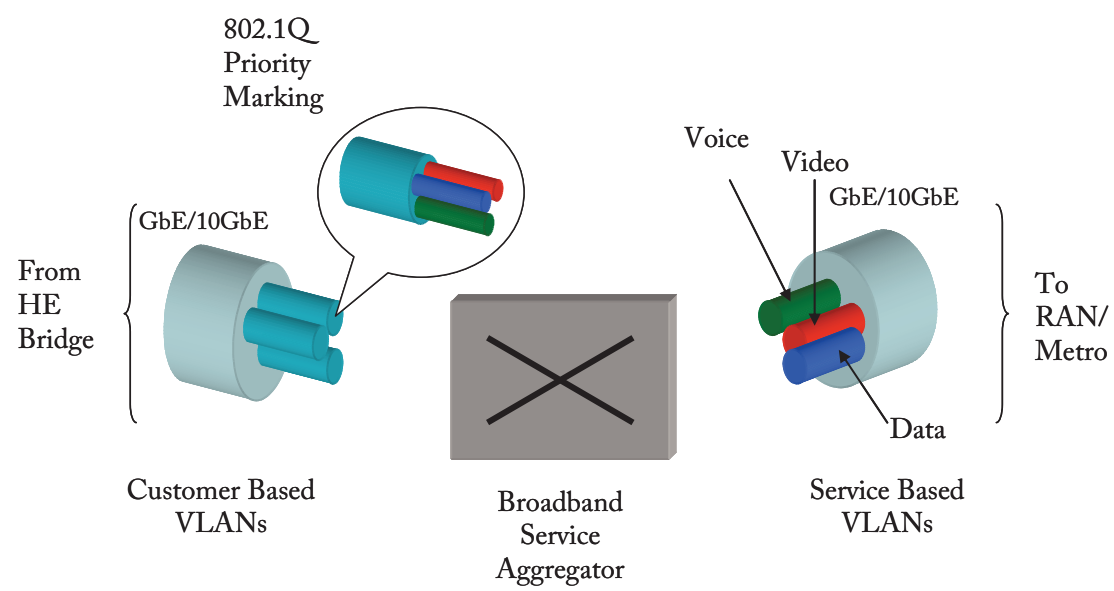

Figure 6-17: Customer based to Service based VLAN translation at BSA

The use of VLANs also avoids direct ONU to ONU communication. With its use, the headend bridge forwarding between ONUs becomes impossible in the entire access network as the IEEE 802.1 specification stipulates that MAC addresses learnt per VLAN can never be forwarded between VLANs even if the DA is known in some other VLAN. The use of VLANs thus also forces intra ONU traffic to be routed via the IP-server of the Service/Content provider which can then be monitored and billed. The architecture allows for the VLAN per subscriber to be viewed as a bandwidth pipe which when used deeper in the network for access aggregation gives per-subscriber traffic flow information to the Broadband Service Aggregators (BSAs). In a service oriented network, the BSAs translate the per-customer VLAN to per-service VLAN for ensuring proper resource dimensioning [70], [71]. A typical schematic of the translation in the network is illustrated in Figure 6-17. 


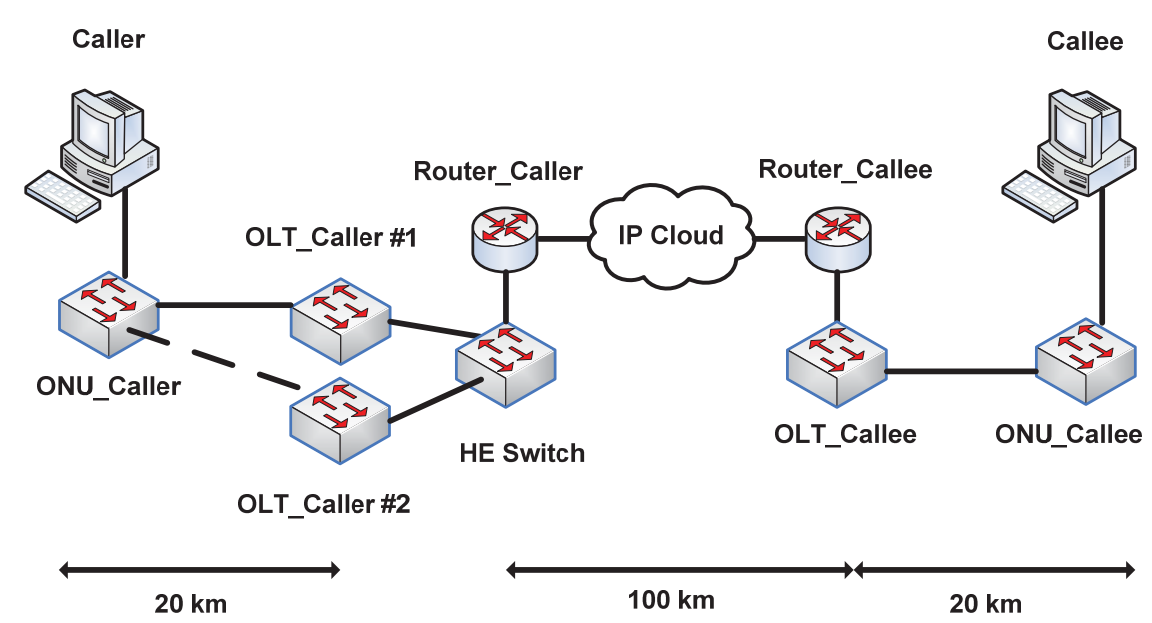

Figure 6-18: Simulation set-up to check MOS degradation with service disruption

The estimation of bandwidth allows for proper dimensioning of bandwidth for service requests. It is no surprise that more bandwidth usually implies a better service delivery [72]. However, the reconfigurability of the network implies there is going to be a finite service disruption whenever it occurs. The performance of real time traffic in presence of disruptions is important to quantify. Figure 6-18 illustrates the set-up used to simulate a VOIP call between two users. A G.711 codec is used to encode voice traffic for transmission between the users. The conversation duration is modelled for 200s. The ONU_Caller is initially associated with OLT_Caller \#1, it is subsequently associated with OLT_Caller \#2 at a simulation time of $100 \mathrm{~s}$. The OLT-ONU distance is taken as a nominal $20 \mathrm{~km}$ for both the caller and the callee, while the public network modelled as a generic IP cloud is taken as $100 \mathrm{~km}$. The traffic flow is not bandwidth restricted or subject to any losses but to disruptions and Figure 6-19 illustrates the Mean Opinion Score (MOS) as a function of disruption time. The MOS in terms of call quality is defined as: $5=$ Excellent, $4=$ Good, $3=$ Fair, 2=Poor, $1=\mathrm{Bad}$. A minimum value of 3 is what is acceptable for a conversation which is not annoying [73]. 


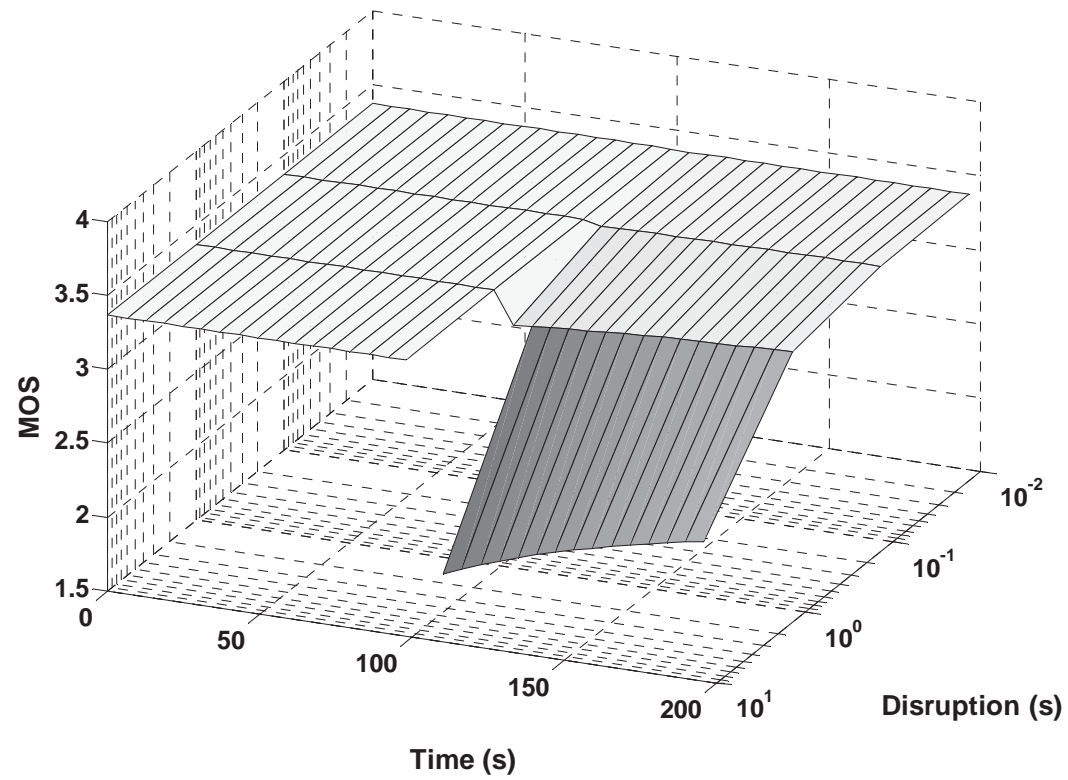

Figure 6-19: MOS as a function of service disruption time

A disruption of $10 \mathrm{~ms}$ is hardly perceptible but with a disruption of $100 \mathrm{~ms}$ the degradation in MOS is more evident. It is however interesting to note that even if the disruption is $1 \mathrm{~s}$, the MOS degradation is not sufficient to make the call quality annoying. Of course if the disruption is longer the MOS drops significantly to make the call quality unacceptable. MOS degradation is more apparent when there are multiple disruptions. Figure 6-20 illustrates the MOS degradation as a function of number of disruptions spaced at $10 \mathrm{~s}$ with each disruption being of $100 \mathrm{~ms}$. The disruptions decrease the overall call quality. Hence an important aspect to consider in the reconfiguration is not only the duration but also the number of disruptions for real time traffic. Typical reconfigurations of less than $100 \mathrm{~ms}$ can be achieved in practical implementations. The simulations were modelled with Opnet Modeler ${ }^{\circledR}$. 


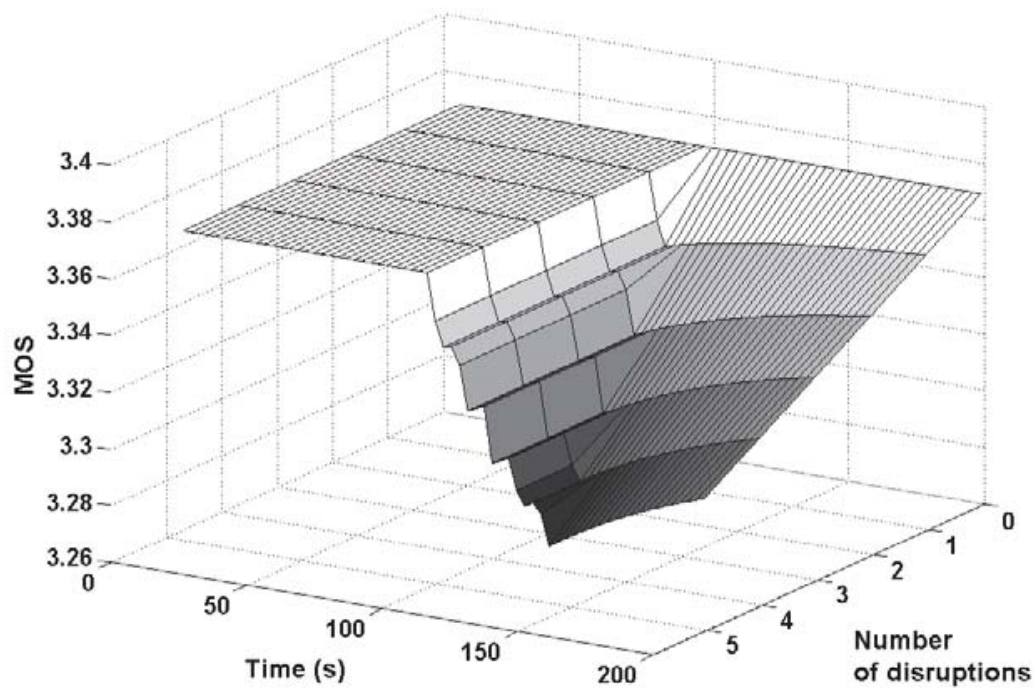

Figure 6-20: MOS as a function of number of disruptions

\subsection{Software and Control Plane perspective}

The management and maintenance of the network is operated in a software platform implemented on a PC at the HE. The elements controlled in the $\mathrm{HE}$ are the following:

- A HE bridge that is used to distribute the downstream traffic over different OLTs and to aggregate the upstream traffic.

- OLTs in the HE to check ONU registrations, allocation of LLIDs to ONUs, and monitoring of multipoint control protocol (MPCP) entities. 


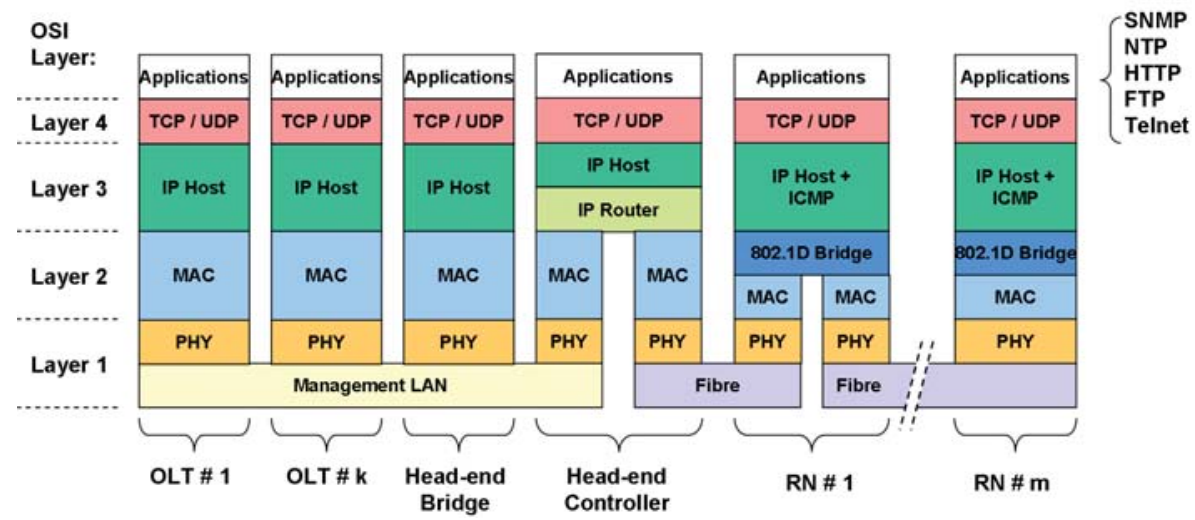

Figure 6-21: Communication protocol stack between HE controller OLTs and HE controller RNs.

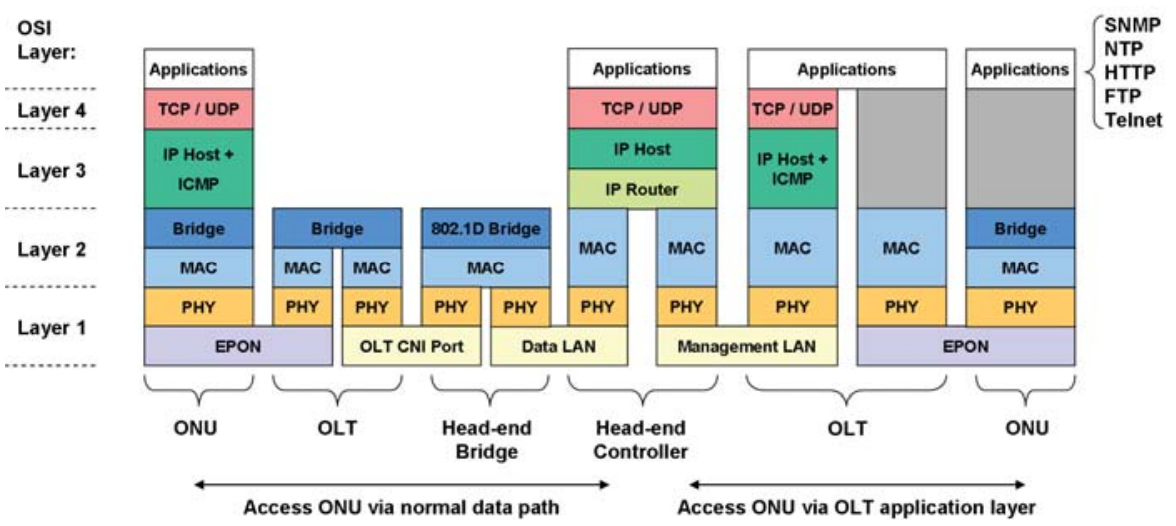

Figure 6-22: Communication protocol stack between OLT and ONUs. 
- Calculation of an optimized network configuration and association of OLTs to ONUs based on bandwidth demand. Maintenance of a resource manager to translate service requests to bandwidth demand requests as an aggregate of VLANs to each of the ONUs.

- Monitoring and configuration of the $\mathrm{RNs}$ to add/drop different wavelengths toward the ONUs depending on the ONU-to-OLT assignment process.

- The maintenance of the LLID-MAC associations of ONUs and migration of these entries once an ONU is served by a different OLT.

- The maintenance of the VLAN to port identification in the HE bridge and migration to another port when the ONU is served by a different OLT.

The HE-based controller can communicate directly with the OLTs and the HEbased switch through a management LAN interface. The HE control and management channel is implementable in an out-of-band bidirectional communication channel based on 1490/1310 nm optics [74]. Figure 6-21 illustrates the communication stack for this. The HE-to-CPE communication is realized through a data plane. A LAN connection is made from the HE controller to one of the traffic ports of the HE bridge, and the management traffic is routed to the ONUs.

The OLTs at the HE also communicate with the associated ONUs via existing MPCP and operations, administration, and maintenance (OAM) protocols and in addition from the OLT application layer to the ONU application layer. Figure 6-22 illustrates the communication stack for this. The management model of the network with $\mathrm{k}$ OLTs, m RNs, and n ONUs is illustrated in Figure 6-23. In this model, each ONU can support one or more connections with a unique identity. The connections are associated with a unique LLID issued by the OLT, a VLAN identifier, and a set of service level agreement (SLA) parameters. In this model each ONU can be associated with multiple end users and each end user can opt for one or more contentservice providers. 


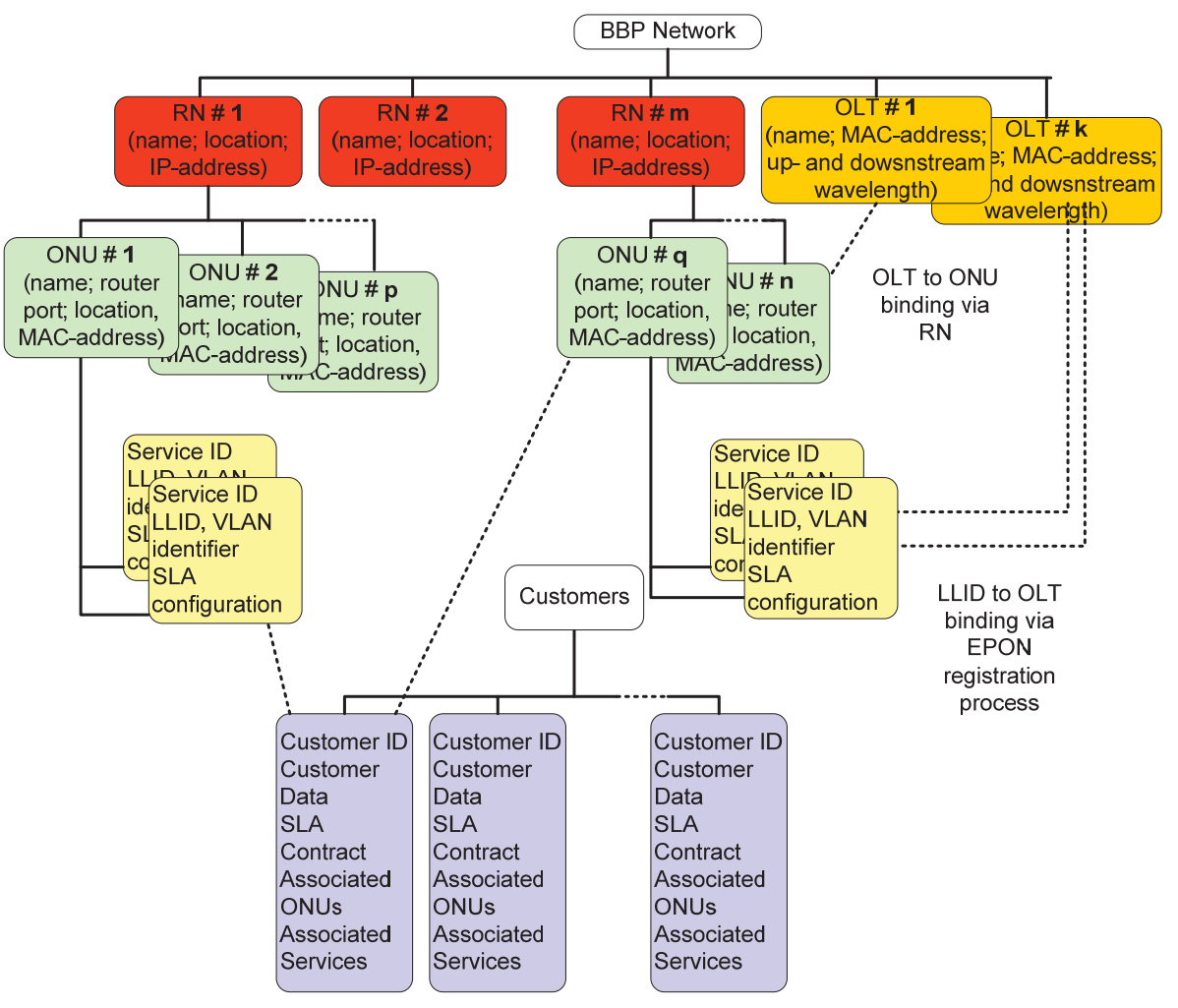

Figure 6-23: Management model of the broadband photonics network.

\subsection{Summary}

This chapter provides an analysis of the service delivery aspects in a BBP like reconfigurable network. Since Ethernet is the most dominant protocol used by a typical home/office user, a perspective view of the network from Ethernet operations has been provided. EPON is assumed to be the bearer protocol and the learning mechanism and operation in a reconfigurable network has been described in detail. It is very clearly shown that depending on the traffic pattern and the scheduling mechanisms in a typical Ethernet switch influences the behaviour of throughput, delay and delay variation in user traffic. The scope of the study was not to detail different scheduling techniques but to emphasise the importance of implementing a proper 
scheme of prioritisation of user traffic to enable facilitation of a good quality of service. To ensure that different classes of service can be implemented, a service architecture model has been designed where the classes of traffic can be defined on basis of VLAN tags. This is based on using the Customer VLAN model as it allows easier monitoring of bandwidth usage per customer. Given the scope of the architecture thus defined, the flow of tagged traffic in such a network has been described. In a reconfigurable network the concern from a service delivery perspective is the disruptive process when an ONU changes association from one OLT to another. In a unique study a typical indication for the quality experienced by a user is the MOS for voice traffic, it is shown that while the duration of disruption is not a major constraint, given the fact that a BBP like network can reconfigure in the order of tens of milliseconds, the other concern is the number of disruptions. It has been indicated in a previous chapter that the methodology to reallocate ONUs to OLTs minimises the number of disruptions for every ONU. The control and management of the network runs over the same network as the flow of data, this perspective view has been described. It ends with a perspective view of the software model used for control and management. 


\section{Chapter 7}

\section{Conclusions and Outlook}

The research work has focussed on aspects related to the operation of the Broadband Photonics (BBP) optical router based dynamically reconfigurable photonic access network. This chapter presents a précis of the achievements of the research work and concludes the thesis with a future outlook for research and development work.

\subsection{Conclusions}

An access network forms a key link in the chain of a network deployment which enables an end user to get what is seen in current times as a ubiquitous resource Bandwidth. For a user the term "Broadband" is often synonymous with the availability of bandwidth. Chapter 1 presents an overview of the evolution of networking technologies associated with the term "Broadband". While traditionally only telco deployments (xDSL, FTTx etc) have been associated with delivery of bandwidth in the access, non telco deployments (HFC, PLC) etc have noticeable to significant presence in some areas. The comparative aspect of the technologies is very clearly elucidated in terms of the way an IP datagram would travel to and fro from the user to the Headend/ Central Office which marks the de-facto interface for an access network with metro/core networks. A network design always forms the key foundation on which bandwidth delivery can be facilitated. The Broadband Photonics net- 
work which is a physical ring providing path redundancy from the Headend to Remote Nodes has been described. It is shown that the network while being a physical ring is logically a P2MP network. The various elements of the network- The Headend with multiple OLTs and provision for out of band control and management channels, the Remote Node with an ROADM subtending multiple CPEs and the CPEs with ONUs have been schematically described. This is used as a basis to understand the scope of operation of the bearer protocols which were considered for the BBP network viz. EPON and GPON.

A network in itself is not capable of providing any service; it merely provides the infrastructure over which other layers are built up to enable a complete communication system. The OSI seven layer model provides a standard framework to reference these various layers. The elements which build up the physical network are described in the Layer 1 or the Physical layer. The Layer 2 or the Data Link Layer protocols demarcates the transition from pure "hardware" to "software" by defining rules of how to access the medium of transmission. Theoretically the different layers are independent of each other, however practically the design of the data link layer is dependent on the physical infrastructure used. One aspect of the research work was to develop an understanding of L2 protocol operation in such a reconfigurable network. Designing a new protocol would be one option but during the course of the work, L2 protocols related to PON operation- the IEEE defined EPON and the ITU-T defined GPON recommendations- were in advanced stages of finalization and commercial deployments conforming initially to draft, and subsequently to published recommendations started seeing a marked increase. Chapter 2 presents a subjective analysis of the EPON protocol. While EPON inherits the legacy of Ethernet, it is different in some aspects. In Ethernet, a network equipment always transmits unless asked not to. In an EPON while the OLT always transmits an ONU has to keep quiet unless asked to transmit. The design and operational parameters of the protocol have been analysed and presented. Chapter 3 continues the analysis of PON based protocols and examines the GPON technology. GPON draws its legacy from SDH and is inherently different from EPON in operation. It 
maintains a $125 \mu$ s framing time reference unlike Ethernet which is a packet based protocol. Despite the technologies being different, they are often compared as both of them are the leading bearer protocols used in commercial PON deployments. A raw bit rate comparison does not do justice to understanding the similarities and differences between the two protocols. Qualitatively both technologies follow the same procedure for operation in a PON environment. At start up the OLT tries to find out which ONUs are connected to it (Discovery) and tries to find the distance at which they are from it (Ranging). In the operational state, the OLT is always broadcasting in the downstream direction while the ONUs are allowed TDM access in the upstream direction. The design and operational parameters of GPON are analysed and compared with respect to EPON.

It is concluded that both EPON and GPON can be used for operation in a BBP like network. Both technologies are addressing the same segment of the network and these can be made operational within the scope of a single network deployment. Thus it can be stated that the technologies are interoperable within the scope of the network deployment. It is however clear that the technologies are independent of each other and they are not inter-operable if the sense of interoperability is defined as associating an EPON OLT with a GPON ONU or the other way around. Both EPON and GPON support transport of Ethernet and TDM traffic. From a service perspective, the bearer L2 protocol can either be EPON or GPON.

Chapter 4 elucidates how the access network can be viewed as a switch between the OLT and ONUs. While a static PON deployment has fixed OLT-ONU association akin to a fixed switch, the BBP network can be viewed as a dynamic switch which allows any OLT-ONU association (if not subject to specific constraints). It explains how the network can be viewed as a logical stack of quasi independent PONs. The concept of bandwidth management on an inter-PON scale is explained. The qualification of having a reconfigurable network is built up by analysing the typical bandwidth requirements of a community and showing with a use case that a reconfigurable network can distribute free capacity in the network where require- 
ment arises. This on the other hand cannot be implemented practically in a static network deployment.

The conclusion which is drawn here is that two things are necessary for promoting the merit of a reconfigurable network. First is that there should be a clear diversity in the bandwidth demand of the users. Second there should be a geographical spread in these users. It is also clear that this statement has been made for the load model considered and while it will be true for any similar distribution, it will have to be qualified for a different type of end user distribution.

A conclusive statement to say that a reconfigurable network is the best solution when there is diversity in the bandwidth demand of end users cannot be made. Given unlimited resources, it is conceptually possible to make a reconfigurable network with existing technology. Without a techno-economic analysis the statement for qualifying the need for a reconfigurable network remains hollow. Within the project scope also there was no successful demonstration of a physical network with more than two end users. Many of the devices required as building blocks for the network including the ROADM which formed an essential element to show-case the reconfigurable network were a failure in terms of performance.

Chapter 5 describes the techniques which can be used to associate ONUs to OLTs to ensure a network configuration where the bandwidth availability to the end user is optimised. The technique is demonstrated on the use case described earlier. A pricing model has been proposed which shows that additional bandwidth can be freed up in the network which can then be used for providing additional bandwidth for existing users or for additional new users. It is shown that this allows a user to "perceive" that the bandwidth available can be up to a factor of two more than the nominal bandwidth available. The key element is of course the diversity in the users and the consequently the disparity in bandwidth requirements. This disparity allows for bandwidth availability which would not otherwise be available if the usage pattern is uniform (assuming network is properly dimensioned without significant overcapac- 
ity). The fact that the network is reconfigurable allows for the redistribution of bandwidth where it is needed. The techniques developed for bandwidth allocation can be extended to any reconfigurable network. The technique developed helps towards operating the network to provide services to users.

It is concluded that useful techniques can be implemented to ensure that proper dimensioning of resources can be done in a BBP like reconfigurable network.

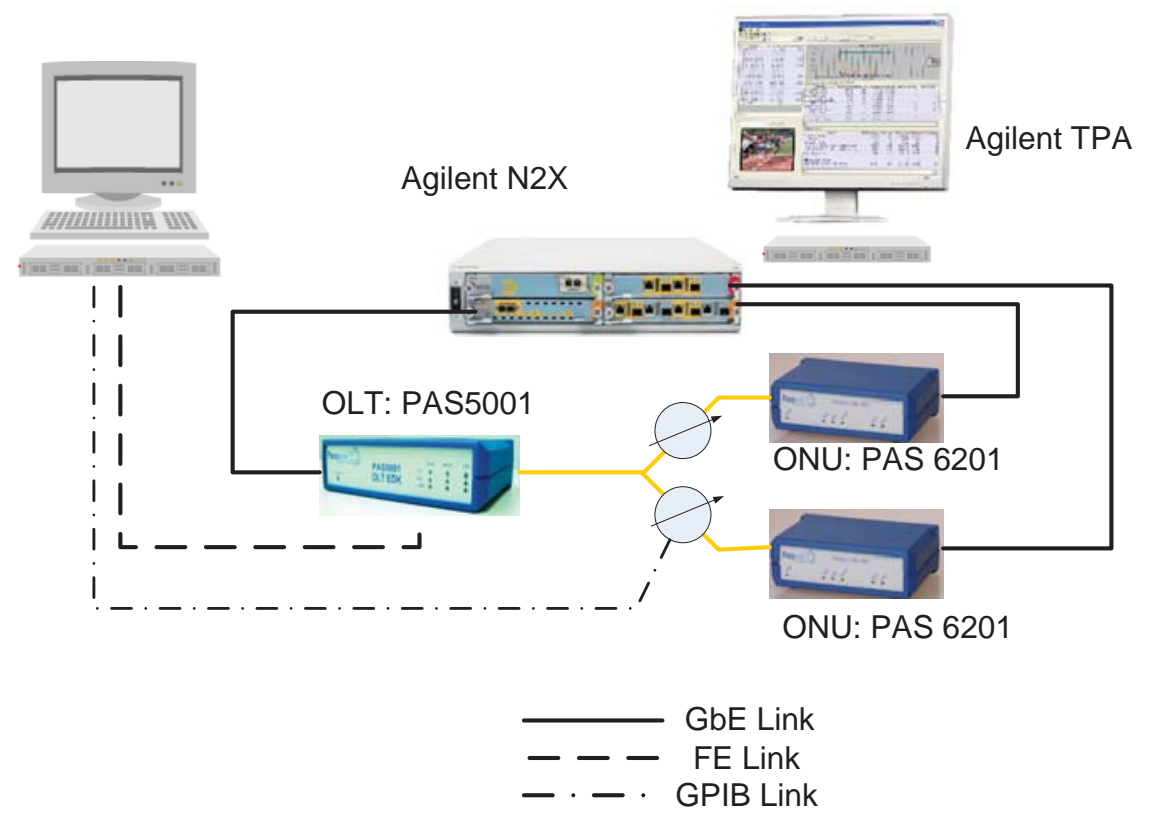

Figure 7-1: Demonstration setup for EPON in a reconfigurable network

As an end-user of the network what one sees is the service; key terms like triple play are common parlance in context of access networks. Applications are becoming more bandwidth intensive than ever. Often bandwidth availability is used as a yardstick as an overall measure of service performance. While availability of bandwidth is a necessary condition to enable services, it is not a sufficient condition to ensure proper service delivery. A comprehensive aspect to design a service delivery platform for 
such a reconfigurable network has been presented in Chapter 6. Perspective views of the network: a data plane perspective as an Ethernet network and a software and control plane perspective have been presented to enable a clear outlook of the service platform. An Agilent N2X platform was used in conjunction with Agilent Triple Play analyser to demonstrate EPON operation in such a reconfigurable network [75]. Figure 7-1 represents a schematic of the setup. At any one time only one of the ONUs was registered with the OLT as the optical signal to the other one was attenuated (below receiver sensitivity) with the VOA. The VOAs thus emulated the functioning of the ROADM where any one of the ONUs could be selectively registered with the only available OLT. It was conclusively shown that it is possible to provide services to an end user in a BBP like reconfigurable network using EPON as a bearer protocol. The non availability of GPON equipment meant that a similar demonstration could not be shown for a case where GPON is the bearer protocol. However based on earlier chapters, it can be conclusively stated that both EPON and GPON can be used as a bearer protocol in a reconfigurable BBP like network.

In Chapter 6, a service architecture has been designed and presented. This architecture supports QoS for multiple classes of service in a network deployment. Further the implementation of a control plane which is independent of the data plane, yet supported in the same network deployment clearly supports the fact that EPON and GPON can be made operational in the network in their native format without any modifications.

It is concluded that a service architecture for delivery of end user services can be designed for a BBP like reconfigurable network. This architecture facilitates QoS for service delivery in the network.

\subsection{Scientific Opinion}

During the scope of the research work, based on the problem statements one can come to certain conclusions. In general while one would want the conclusions to be 
"conclusive", it is not always the case. The discussion on whether there can be an "inconclusive" conclusion falls within the realm of philosophy. While it is not the scope of this manuscript to extend discussion in that area, it might be of interest to hazard some guesses for the "inconclusive" conclusions. Under what circumstances can a reconfigurable network be a good choice is a pertinent one to examine.

Can the perceived bandwidth be made significantly higher? In general this will depend on the duration of the "busy hour" and the acceptable block probability. However, when the demands increase, it becomes more difficult to free up available bandwidth for use. For the given use case or similar configurations, it is very unlikely that the perceived bandwidth can be made significantly higher than a factor of two, compared to a static network, if the number of users who need the increased bandwidth remains the same. This factor will only reduce if there is more uniformity in the bandwidth demand of the users. However, if the number of users who need the excess bandwidth reduces, for instance, instead of a quarter of the users, if only one eighth of the users need excess bandwidth, the perceived bandwidth can be scaled up to a factor higher than four.

It has been shown that disparity in the demands of the users is important to ensure that there is some bandwidth which can be made available to users who require the additional bandwidth. With a homogeneous residential base, the temporal demand pattern will be similar to each other and there will not be much benefit for a reconfigurable network if the number of users is increased. Can the number of users be significantly higher? The answer to this is yes. It can be argued that such a network can support up to twice the number of users in comparison to a static network if only the temporal demands of about half of them are peaking at a different time than from the other half. This typically would be the case if half the number of users were commercial and not residential.

Is a reconfigurable network deployment then better than a static deployment? In general, yes. To set an analogy, it is like saying the whole is more than the sum of 
the parts. However, this needs to be quantified in terms of the efforts required to implement such a network and the technical feasibility to do so.

\subsection{Outlook}

Like every project executed, the BBP project has a natural lifespan and the project has drawn to a close. Significant advancements in various topics have been made during the course of the project. The knowledge thus acquired, both of the achievements and limitations, also helps in building foundations to stimulate and nurture further research.

Commercial feasibility of engineering research projects is important to ensure techno-economic viability and success of such projects. This area should form an interesting field of study and could project interesting comparison in the direction of evolution of access networks. What a BBP like network can do is to optimise resource delivery to the end user, this in turn facilitates optimal performance for the end-user. Technically similar performance in a network can also be achieved without optimisation by: a) increasing the infrastructure or b) by increasing the data rate supported by the network or a combination thereof. A techno-economic study will provide information to answer key questions on what is required to make the network commercially viable:

- What is the element of reconfigurability required in the network?

- What should be the target range of access network and the type of user base to be supported?

- What kind of alternative technologies would be viable to implement the platform?

Further research on bandwidth re-distribution techniques can be implemented to add another dimension to reconfigurability. For instance if EPON is used as the bearer PON protocol of operations, techniques like Link Aggregation can be im- 
plemented in such a network which enables increased bandwidth delivery and an added resiliency in the network [76]. Areas of research open in this field include:

- Study of architecture for service delivery including operation of Link Aggregation in a BBP like network.

- Development of similar techniques to realise port aggregation for operation of GPON as the base PON protocol can be researched and developed.

The techniques developed for bandwidth reconfiguration are novel and not constrained to a BBP like network only. Further studies can include focus on use of the techniques developed in:

- Similar reconfigurable network architectures based on other media like wireless access.

- Domains which are similar to access in terms of forming a segment which facilitates bandwidth delivery as in in-house communications or in data acquisition systems for industrial use.

Access networks continue to get a lot of focus in industry and academia. While fiber deployment has continued to see growth in deployment, not only because of the obvious advantages it offers, but also because the component technologies are evolving in parallel to make components cheaper without compromising on performance. The debate of copper versus fiber usually leaves out a key player which has evolved as an important competitor to fiber based access network viz. Broadband Wireless access. Providing mobility is the key strong point of such technologies and these can be used as complimentary rather than competing technologies. Further study can include

- Convergence of wireless access technologies with a BBP like network to develop pseudo-static or limited mobility access networks. 
Both IEEE and ITU-T are in the process of defining NG2 PON systems. The use of $10 \mathrm{~Gb} / \mathrm{s}$ technology is commonly referred to as Next Generation 1 or NG1 and the evolution from there on is seeing a split. On one hand the development is targeting higher speed ( 40 or $100 \mathrm{~Gb} / \mathrm{s}$ ) systems while on the other hand there is the use of keeping the line rates the same $(10 \mathrm{~Gb} / \mathrm{s})$ but using multiple wavelengths. The latter is referred to as the Next Generation 2 or NG2 technology. Further study can include

- Enhancing the techniques used for a BBP like network for use in NG2 neworks. 


\section{References}

[1] ITU-T V.90, "A digital modem and analogue modem pair for use on the Public Switched Telephone Network (PSTN) at data signalling rates of up to $56000 \mathrm{bit} / \mathrm{s}$ downstream and up to $33600 \mathrm{bit} / \mathrm{s}$ upstream”, ITU-T Standard.

[2] ITU-T V.92, "Enhancements to Recommendation V.90", ITU-T Standard.

[3] ITU-T I.412, "ISDN user-network interfaces - Interface structures and access capabilities", ITU-T Standard.

[4] ITU-T G.990-999, “Access Networks”, ITU-T Standards.

[5] ITU-T J.112, "Transmission systems for interactive cable television services", ITU-T Standard.

[6] ITU-T J.122, "Second-generation transmission systems for interactive cable television services - IP cable modems", ITU-T Standard.

[7] ITU-T J.222.1-3, "Third-generation transmission systems for interactive cable television services", ITU-T Standards.

[8] "Leveraging RFoG to Deliver DOCSIS ${ }^{\circledR}$ and GPON Services Over Fiber", White Paper (2008), www.motorola.com.

[9] "Fixed and wireless broadband subscriptions by technology", OECD Broadband Portal (June 2010), www.oecd.org

[10] "Fixed and wireless broadband subscriptions per 100 inhabitants (June 2010)", OECD Broadband Portal, www.oecd.org.

[11] Teresa Mastrangelo, "Global FTTH Deployment Trends; Which Architecture Makes the Most Sense and Why?" (2007), www.broadbandtrends.com

[12] A. Banerjee, Y. Park, F. Clarke, H. Song, S. Yang, G. Kramer, K. Kim, and B. Mukherjee, "Wavelength-division-multiplexed passive optical network (WDM-PON) technologies for broadband access: a review [Invited]," J. Opt. Netw. 4, 737-758 (2005).

[13] "Frost \& Sullivan: bandwidth demand, future proofing driving optical deployments globally," S. M. Hardy, ed., Lightwave Direct Newsletter, May 2008, www.lw.pennet.com. 
[14] IEEE 802.3av-2009, "Carrier sense multiple access with collision detection (CSMA/CD) access method and physical layer specifications, Amendment 1: Physical Layer Specifications and Management Parameters for $10 \mathrm{~Gb} / \mathrm{s}$ Passive Optical Networks" IEEE Standard.

[15] Freeband project website, www.freeband.nl

[16] P. Urban, E. J. Klein, L. Xu, E. G. C. Pluk, A. M. J. Koonen, G. D. Khoe, and H. de Waardt, "1.25-10 Gbit/s reconfigurable access network architecture," in Proceedings of the 9th International Conference on Transparent Optical Network (IEEE, 2007), pp. 293-296.

[17] R. Roy and W. van Etten, "Design of a survivable multi-wavelength photonic access network," in Proceedings of the 2nd International Conference on Access Networks (IEEE, 2007), pp. 1-6.

[18] R.Roy, G. Manhoudt, C.G.H Roeloffzen and W. van Etten, "Control and management scheme in a DWM EPON", in Proceedings of the $8^{\text {th }}$ International Conference on Transparent Optical Networks (IEEE), 18-22 June 2006, Nottingham, United Kingdom.

[19] R. Teune, R.Roy and W. van Etten, "Demonstration of IP based control and management for a reconfigurable photonic access network", in Proceedings of $10^{\text {th }}$ International Conference on Transparent Optical Networks (IEEE), 22-26 June 2008, Athens, Greece.

[20] E. Klein, P. Urban, G. Sengo, L. T. Hilderink, M. Hoekman, R. Pellens, P. van Dijk, and A. Driessen, "Densely integrated microring resonator based photonic devices for use in access networks," Opt. Express 15, 10345-10355 (2007).

[21] IEEE 802.3-2008, Section 5, "Carrier sense multiple access with collision detection (CSMA/CD) access method and physical layer specifications," IEEE Standard.

[22] IEEE 802.3-2008, Section 2, "Carrier sense multiple access with collision detection (CSMA/CD) access method and physical layer specifications," IEEE Standard. 
[23] IEEE 802.1Q-2005, "Virtual Bridged Local Area Networks," IEEE Standard.

[24] Glen Kramer and Biswanath Mukhherjee, "IPACT: A Dynamic Protocol for an Ethernet PON (EPON)," IEEE Commun. Mag., vol. 42, no. 2, pp. 7480, (2002)

[25] M.P. McGarry, M. Maier, and M. Reisslein, “Ethernet PONs: A Survey of Dynamic Bandwidth Allocation (DBA) Algorithms", IEEE Commun. Mag., vol 42, no.8, S8-S15 (2004)

[26] G. Kramer, Ethernet Passive Optical Networks (McGraw-Hill, 2005), pp 201-213.

[27] G. Kramer and K. Tanaka, "Advances in Optical Access Networks", in Proceedings of the Optical Fiber Communications Conference, 2005, Technical Digest (IEEE, 2005), Vol. 4, pp. 1-3

[28] ITU-T G.983.1-3, "Broadband optical systems based on passive optical networks (PON), ITU-T Standards.

[29] ITU-T G.984.1-6, “Gigabit capable passive optical networks”, ITU-T Standards.

[30] "GPON versus EPON: the battle lines are drawn”, R.Rubenstein, FiberSystems Europe (fibersystems.org), December 2005.

[31] Frank J. Effenberger, Kent McCammon, and Vincent O'Byrne, "Passive optical network deployment in North America [Invited]," J. Opt. Netw. 6, 808818 (2007)

[32] Hiromichi Shinohara, "FTTH experiences in Japan [Invited]," J. Opt. Netw. 6, 616-623 (2007)

[33] www.teknovus.com

[34] ITU-T G.982, “ Optical Access Networks to support services up to ISDN primary rate or equivalent rates", ITU-T Standard.

[35] "Despite economics, WDM-PON remains in the mix", M. Fuller Hanna., Lightwave Direct Newsletter (lw.pennet.com), August 2008 
[36] "WDM PON: How long is it going to take" (2009), A. Najam, www.frost.com

[37] Chang-Hee Lee, Sang-Mook Lee, Ki-Man Choi, Jung-Hyung Moon, SilGu Mun, Ki-Tae Jeong, Jin Hee Kim, and Byoungwhi Kim, "WDM-PON experiences in Korea [Invited]," J. Opt. Netw. 6, 451-464 (2007).

[38] A.X. Widmer, P.A. Franaszek, "A DC-Balanced, Partitioned-Block, 8B/ 10B Transmission Code", IBM J. Res. Develop. 27(5), 440-451 (1983).

[39] L. B. James, A. W. Moore, R. Plumb, M. Glick, A. Wonfor, I. H. White, D. McAuley, R. V. Penty, "Beyond Gigabit Ethernet: Physical Layer Issues in Future Optical Networks", London Communications Symposium (2004).

[40] "GPON-The Next Big Thing in Optical Access Networks", www.flexlightnetworks.com

[41] ITU-T G.707/Y.1322, "Network Node Interface for the Synchronous Digital Hierarchy (SDH)", ITU-T Standard.

[42] B.G. Lee and S.C. Kim, “ Low-Rate Parallel Scrambling Techniques for Today's Lightwave Transmission”, IEEE Commun. Mag., vol. 33, no. 4, pp. 84-95, (1995).

[43] Cedric Mélange, Bart Baekelandt, Johan Bauwelinck, Peter Ossieur, Tine De Ridder, Xing-Zhi Qiu, and Jan Vandewege, "Burst-Mode CDR Performance in Long-Reach High-Split Passive Optical Networks," J. Lightwave Technol. 27, 3837-3844 (2009)

[44] R.P. Davey, P. Healey, I. Hope, P. Watkinson and D.B. Payne, O Marmur, J. Ruhmann, Y. Zuderveld, "DWDM reach extension of a GPON to 135 km" J. Lightwave Technol. 24, 29-31 (2006)

[45] ITU-T G.984.6, "Gigabit capable passive optical networks: Reach Extension”, ITU-T Standard.

[46] ITU-T G.984.7, "Gigabit capable passive optical networks: Long Reach", ITU-T draft.

[47] B. Skubic, J. Chen, J. Ahmed, L. Wosinska and B. Mukherjee, “ A Comparison of Dynamic Bandwidth Allocation for EPON, GPON and Next 
Generation TDM PON", IEEE Commun. Mag., vol 47, no.3, S40-S48 (2009).

[48] "Dell'Oro: GPON sales surge in 2010 first quarter, surpass EPON", Lightwave Direct Newsletter (lw.pennet.com), June 2010.

[49] K. Fukuda, K. Cho, and H. Esaki, "The impact of residential broadband traffic on Japanese ISP backbones," Comput. Commun. Rev. 35, 15-21 (2005).

[50] Broadband Stakeholder Group, "Predicting UK future residential bandwidth requirements" (May 2006), www.broadbanduk.org.

[51] Amsterdam Internet Exchange, www.ams-ix.net.

[52] Munich Internet Exchange, www.inxs.de.

[53] G. Kramer, Ethernet Passive Optical Networks (McGraw-Hill, 2005), pp. 173-181.

[54] J. Kallrath and J.M. Wilson, Business Optimization: Using Mathematical Programming (Macmillan Business, 1997).

[55] J. Bisschop, “AIMMS: optimization modelling (2007)," www.aimms.com.

[56] D. Banerjee and B. Mukherjee, "A Practical Approach for Routing and Wavelength Assignment in Large Wavelength-Routed Optical Networks", IEEE JSAC., vol. 14, no. 5, pp. 903-908 (1996).

[57] H. Zhang, J.P. Jue and B. Mukherjee, " A Review of Routing and Wavelength Assignment Approaches for Wavelength Routed Optical WDM Networks", Optical Networks Mag., pp. 47-60 (2000).

[58] V. Souza, B. Tremblay, P. Karlsson, J. R. I. Riu, K. Persson, Z. Ghebretensae, and W. Zhao, "A QoS enabled public ethernet access network," in Proceedings of the 12th European Conference on Networks and Optical Communications, D. W. Faulkner, R. Neat, and E.Vannin, eds. (Acreo AB), 19-21 June 2007, Stockholm, Sweden.

[59] A. Elmaghraby, A. Kumar, M. M. Kantardzic, and M. G. Mostafa, "Bandwidth allocation in a dynamic environment using a variable pricing policy," in Proceedings of the 7th International Symposium of Computers and Communications (IEEE, 2002), pp. 589-594. 
[60] A. Erramilli, E. H. Lipper, and J. L. Wang, "Some performance considerations for mass market broadband services," in Proceedings of the 1st International Workshop on Community Networking Integrated Multimedia Services to the Home (IEEE, 1994), pp. 109-116.

[61] Y. Lu, F. Kuipers, M. Janic, and P. V. Mieghem, "E2E blocking probability of IPTV and P2PTV," in Proceedings of the 7th International Conference on Networking (IFIP, 2008), pp. 445-456.

[62] P. van Mieghem, "Queuing models," in Performance Analysis of Communications Networks and Systems (Cambridge, 2006), pp. 279-282.

[63] IEEE 802.1D-2004, "Media Access Control (MAC) Bridges," IEEE Standard.

[64] Chen-Chi Wu, Kuan-Ta Chen, Yu-Chun Chang and Chin-Laung Lei, "Detecting VoIP Traffic Based on Human Conversation Patterns",Principles, Systems and Applications of IP Telecommunications. Services and Security for Next Generation Networks, Lecture Notes in Computer Science, 280-295 (2008).

[65] Jeongeun Julie Lee and Maruti Gupta, “ A New Traffic Model for Current User Web Browsing Behavior", Intel Research (2007), www.intel.com

[66] TR-101, Release 3.0, " Migration to Ethernet based DSL aggregation, “ Technical Report (2006), www.broadbandforum.org

[67] TR-156, Issue 2, "Using GPON Access in the context of TR-101", Technical Report (2010), www.broadbandforum.org

[68] TR-200, Issue 1, "Using EPON Access in the context of TR-101", Technical Report (2011), www.broadbandforum.org

[69] Marc Bernstein, “VLAN design for IPTV networks,”, White paper (2010), Juniper Networks.

[70] "Alcatel Triple Play Service Delivery Architecture", Technology White Paper(2007), Alcatel Lucent.

[71] Alan Sardella, " Optimizing Broadband Service Delivery", White paper (2006), Juniper Networks. 
[72] R.Roy, G. Manhoudt and W. van Etten, "Service delivery aspects in a reconfigurable photonic access network", in Proceedings of 13th European Conference on Networks E Optical Communications E Optical Cabling and Infrastructure, 1-3 July 2008, Krems, Austria

[73] ITU-T P.800, "Methods for subjective determination of transmission quality", ITU-T Standard.

[74] R. Teune, R. Roy, and W. van Etten, "Demonstration of IP based control and management for a reconfigurable photonic access network," in Proceedings of 10th International Conference on Transparent Optical Networks (IEEE, 2008), pp. 145-148, 22-26 June 2008, Rome, Italy

[75] R. Roy, "Reconfigurability in an Access Network: A BBP Perspective”, BBP Workshop, 18th April 2008, Eindhoven.

[76] R. Roy, G. Manhoudt, and W. van Etten, "Link Aggregation in a MultiWavelength Reconfigurable Photonic Access Network," in Proceedings of Access Networks and In-house Communications, (OSA, 2010) , 21-24 June 2010, Karlsruhe, Germany.

[77] Generalised Pareto Distribution, Matlab R2012b Documentation, www.mathworks.nl 


\section{Appendix A}

The generalised Pareto distribution for location parameter $\mu$, shape parameter $\xi$, and scale parameter $\sigma$ is defined by [77]:

$$
f(\xi, \mu, \sigma)(x)=\frac{1}{\sigma}\left(1+\frac{\xi(x-\mu)}{\sigma}\right)^{\left(\frac{-1}{\xi}-1\right)}
$$
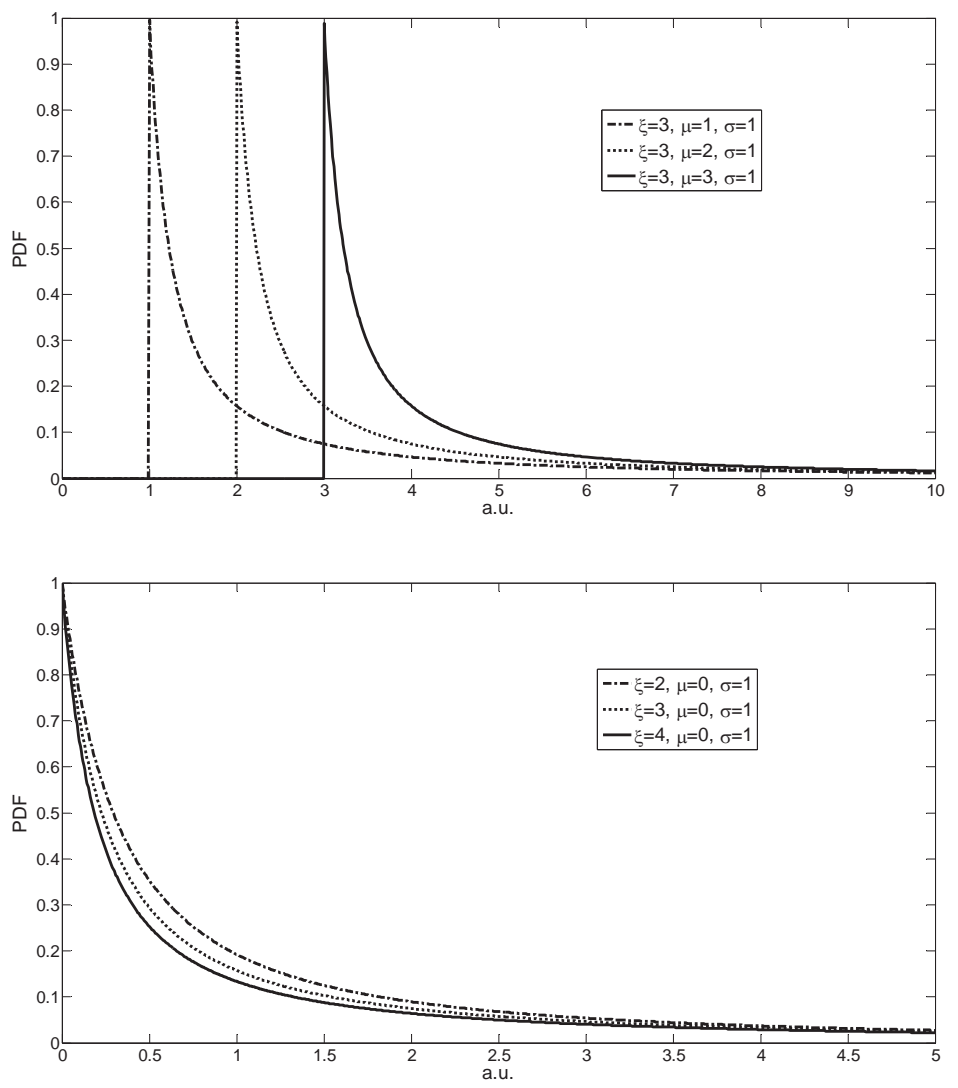


\section{Appendix B}

For an $\mathrm{M} / \mathrm{M} / \mathrm{m} / \mathrm{m} / \mathrm{s}$ queue ( a queue with exponentially distributed arrival and processing times without any buffer capacity for customers to wait) with a mean arrival rate of $\lambda$ and an exponentially distributed hold time of $1 / \mu$ with $m$ servers and $s$ customers, the service blocking probability $P_{B}$ is given by [62]:

$$
P_{B}=\frac{\frac{(s-1) !}{m !(s-1-m) !}\left(\frac{\lambda}{\mu}\right)^{m}}{\sum_{n=0}^{m} \frac{(s-1-m) !}{n !(s-1-n) !}\left(\frac{\lambda}{\mu}\right)^{n}}
$$




\section{Acronyms}

$\begin{array}{ll}\text { ADSL } & \text { Asymmetric DSL } \\ \text { APON } & \text { ATM PON } \\ \text { ATM } & \text { Asynchronous Transfer Mode } \\ \text { ATU-R } & \text { ADSL Termination Unit-Remote } \\ \text { BBP } & \text { Broadband Photonic } \\ \text { BIP } & \text { Bit Interleaved Parity } \\ \text { BPON } & \text { Broadband Passive Optical Network } \\ \text { BRI } & \text { Basic Rate Interface } \\ \text { BSA } & \text { Broadband Service Aggregator } \\ \text { CATV } & \text { Cable Television } \\ \text { CD } & \text { Compact Disc } \\ \text { CDR } & \text { Clock Data Recover } \\ \text { CIF } & \text { Common Intermediate Format } \\ \text { CM } & \text { Cable Modem } \\ \text { CMTS } & \text { Cable Modem Termination System } \\ \text { CPE } & \text { Customer Premises Equipment } \\ \text { CW } & \text { Continuous Wave } \\ \text { CWDM } & \text { Coarse WDM } \\ \text { DA } & \text { Destination Address } \\ \text { DAB } & \text { Digital Audio Broadcasting } \\ \text { DBA } & \text { Dynamic Bandwidth Allocation } \\ \text { DBR } & \text { Dynamic Bandwidth Report } \\ \text { DEI } & \text { Drop Eligibility Indication } \\ \text { DOCSIS } & \text { Data Over Cable Service Interface } \\ \text { DS } & \text { Downstream } \\ \text { DSL } & \text { Digital Subscriber Line } \\ \text { DSLAM } & \text { Digital Subscriber Line Access Multiplexer } \\ \text { DWDM } & \text { Dense WDM } \\ & \end{array}$


EFM

EPON

FCFS

FE

FIFO

FSAN

FSS

FTTx

$\mathrm{GbE}$

GEM

GPON

GTC

HDLC

HDSL

HDTV

HE

HEC

HFC

HFW

HTTP

IAT

IDSL

IEEE

IP

ISDN

ITU

LLID

LOF

LOS

LP

MAC
Ethernet First Mile

Ethernet Passive Optical Network

First Come First Serve

Fast Ethernet

First in First Out

Full Service Access Network

Frame Synchronous Scrambling

Fiber To The House/Building/Curb

Gigabit Ethernet

GPON Encapsulation Method

Gigabit Capable Passive Optical Network

GPON Transmission Convergence Layer

High level Data Link Control

High data rate DSL

High Definition Television

Headend

Header Error Control

Hybrid Fiber Coax

Hybrid Fiber Wireless

Hyper Text Transfer Protocol

Inter Arrival Time

ISDN DSL

Institution of Electrical and Electronic Engineers

Internet Protocol

Integrated Services Digital Network

International Telecommunication Union

Logical Link Identifier

Loss of Frame

Loss of Signal

Linear Programming

Medium Access Control 


$\begin{array}{ll}\text { MAN } & \text { Metropolitian Area Network } \\ \text { MLM } & \text { Multi Longitudinal Mode } \\ \text { MoCA } & \text { Multimedia over Coax Association } \\ \text { MOS } & \text { Mean Opinion Score } \\ \text { MPCPDU } & \text { Multipoint Control Protocol Data Unit } \\ \text { NGA } & \text { Next Generation Access } \\ \text { OAM } & \text { Operations Administration and Management } \\ \text { ODN } & \text { Optical Distribution Network } \\ \text { OECD } & \text { Organisation for Economic Co-operation and De- } \\ & \text { velopment } \\ \text { OLT } & \text { Optical Line Termination } \\ \text { ONU } & \text { Optical Network Unit } \\ \text { OSI } & \text { Open System Interconnect } \\ \text { P2MP } & \text { Point to Multipoint } \\ \text { P2P } & \text { Point-to-point } \\ \text { PCB } & \text { Physical Control Block } \\ \text { PDV } & \text { Packet Delay Variation } \\ \text { PLC } & \text { Power Line Communication } \\ \text { Plen } & \text { Payload Length } \\ \text { PLI } & \text { Payload Indication } \\ \text { PLO } & \text { Physical Layer Overhead } \\ \text { PLOAM } & \text { Physical Layer OAM } \\ \text { PON } & \text { Passive Optical Network } \\ \text { POTS } & \text { Plain Old Telephone System } \\ \text { PPP } & \text { Point-to-point Protocol } \\ \text { PQ } & \text { Priority Queue } \\ \text { PRI } & \text { Primary Rate Interface } \\ \text { PSTN } & \text { Public Switched Telephone Network } \\ \text { Psync } & \text { PTI } \\ \text { QoS } & \text { Paylity of Service } \\ & \end{array}$




\begin{tabular}{|c|c|}
\hline RFoG & Radio Frequency over Glass \\
\hline RG & Residential Gateway \\
\hline $\mathrm{RN}$ & Remote Node \\
\hline ROADM & Reconfigurable Optical Add/Drop Multiplexer \\
\hline $\mathrm{RR}$ & Round Robin \\
\hline RS & Reconcilliation Sublayer \\
\hline RSOA & Reflective Semiconductor Optical Amplifier \\
\hline SA & Source Address \\
\hline SAR & Segmentation and Reassembly \\
\hline $\mathrm{SDH}$ & Synchronous Digital Hierarchy \\
\hline SDSL & Symmetric DSL \\
\hline SDTV & Standard Definition Television \\
\hline SHDSL & Symmetric HDSL \\
\hline SLM & Single Longitudinal Mode \\
\hline T-CONT & Transport Container \\
\hline $\mathrm{TC}$ & Transmission Convergence \\
\hline TDM & Time Division Multiplexing \\
\hline TELCO & Telecommunication \\
\hline TPID & Tag Protocol Identifier \\
\hline TQ & Time Quanta \\
\hline TU & Tributary Unit \\
\hline UART & Universal Asynchronous Receiver/ Transmitter \\
\hline US & Upstream \\
\hline USB & Universal Serial Bus \\
\hline VDSL & Very high bit rate DSL \\
\hline VLAN & Virtual Local Area Network \\
\hline VID & VLAN Identifier \\
\hline VOA & Variable Optical Attenuator \\
\hline WAN & Wide Area Network \\
\hline WDM & Wavelength Division Multiplexing \\
\hline WRR & Weighted Round Robin \\
\hline
\end{tabular}




\section{List of Publications}

Journals

- R.Roy, G. Manhoudt and W. van Etten, "Optical-router based dynamically reconfigurable photonic access network", J. Opt. Netw. 8(1), 51-76 (2009)

- P.J.Urban, B.Huiszoon, R.Roy, F.M. Huijskens, E.J.Klein, G.D. Khoe. A.M.J. Koonen and H. de Waardt, “ High bitrate reconfigurable WDM ring shaped access network", J. Opt. Comm. And Netw. 1(2), A143-A159 (2009)

Conferences

- R.Roy, G. Manhoudt, C.G.H Roeloffzen and W. van Etten, "Control and management scheme in a DWDM EPON", in Proceedings of the $8^{\text {th }}$ International Conference on Transparent Optical Networks (IEEE), 18-22 June 2006, Nottingham, United Kingdom

- R.Roy, C.G.H Roeloffzen and W. van Etten, "Switched DWDM Ethernet Passive Optical networks", in Proceedings of the $11^{\text {th }}$ Annual Symposium IEEE/LEOS Benelux, (IEEE), 30 Nov- 1 Dec 2006, Eindhoven, the Netherlands

- $\quad$ R.Roy, G. Manhoudt and W van Etten, "Performance characterization of a multi-wavelength Photonic Access Network with a use case scenario", in Proceedings of the $12^{\text {th }}$ European Conference on Networks and Optical Communications, (Acreo AB), 19-21 June 2007, Stockholm, Sweden

- R.Roy, G. Manhoudt and W. van Etten, "Bandwidth Re-Distribution Techniques for Extended EPON Based Multi-Wavelength Networks", in Proceedings of the $9^{\text {th }}$ International Conference on Transparent Optical Networks (IEEE), 1-5 July 2007, Rome, Italy

- R.Roy and W. van Etten, "Design of a survivable multi-wavelength photonic access network", in Proceedings of the $2^{\text {nd }}$ International Conference on Access Networks (IEEE), 22-24 August 2007, Ottawa, Canada 
- R.Roy, G. and W. van Etten, "Provision of Metro Ethernet Services using a reconfigurable photonic access netowrk", in Proceedings of the $12^{\text {th }}$ Annual Symposium IEEE/LEOS Benelux, (IEEE), 17-18 Dec 2007, Brussels, Belgium

- R. Teune, R.Roy and W. van Etten, "Demonstration of IP based control and management for a reconfigurable photonic access network", in Proceedings of $10^{\text {th }}$ International Conference on Transparent Optical Networks (IEEE), 22-26 June 2008, Athens, Greece

- R.Roy, G. Manhoudt and W. van Etten, "Service delivery aspects in a reconfigurable photonic access network", in Proceedings of the $13^{\text {th }}$ European Conference on Networks and Optical Communications, 1-4 July 2008, Krems, Austria

- $\quad$ R.Roy and W. van Etten, "Case for Dynamic Reconfigurability in Access Networks", in Proceedings of the $3^{\text {rd }}$ International Conference on Access Networks (ICST, Springer), 15-17 October 2008, Las Vegas, USA

- R. Roy, G. Manhoudt, and W. van Etten, "Link Aggregation in a MultiWavelength Reconfigurable Photonic Access Network," in Proceedings of the Access Networks and In-house Communications, (OSA), 21-24 June 2010, Karlsruhe, Germany

Others

- $\mathrm{R}$, Roy, "Bandwidth Reallocation techniques in reconfigurable photonic access networks", Short Term Scientific Mission (STSM), COST-293, 413 June 2007, University of Sussex, Sussex, UK. 